\title{
Guided Waves in Thin-Walled Structural Members.
}

\author{
A Thesis \\ Presented to \\ the Department of Civil and Geological Engineering \\ Faculty of Engineering \\ The University of Manitoba
}

\begin{abstract}
In Partial Fulfillment
of the Requirements for the Degree of

Master of Science in Civil Engineering
\end{abstract}

by

John B.C. Rogers

April 1999 
Acquisitions and Bibliographic Services

395 Weillington Street Ottawa ON K1A ON4 Canada
Bibliothèque nationale du Canada

Acquisitions et services bibliographiques

395, rue Wellington

Ottawa ON K1A ON4

Canada
The author has granted a nonexclusive licence allowing the National Library of Canada to reproduce, loan, distribute or sell copies of this thesis in microform, paper or electronic formats.

The author retains ownership of the copyright in this thesis. Neither the thesis nor substantial extracts from it may be printed or otherwise reproduced without the author's permission.
L'auteur a accordé une licence non exclusive permettant à la Bibliothèque nationale du Canada de reproduire, prêter, distribuer ou vendre des copies de cette thèse sous la forme de microfiche/film, de reproduction sur papier ou sur format électronique.

L'auteur conserve la propriété du droit d'auteur qui protège cette thèse. $\mathrm{Ni}$ la thèse ni des extraits substantiels de celle-ci ne doivent être imprimés ou autrement reproduits sans son autorisation. 
THE UNIVERSITY OF MANITOBA

FACULTY OF GRADUATE STUDIES

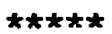

COPYRIGHT PERMISSION PAGE

GUIDED WAVES IN THIN-WALLED STRUCTURAL MEMBERS.

BY

JOHN B. C. ROGERS

A Thesis/Practicum submitted to the Faculty of Graduate Studies of The University of Manitoba in partial fulfilmment of the requirements of the degree

of

MASTER OF SCIENCE

JOHN B. C. ROGERS $\odot 1999$

Permission has been granted to the Library of The University of Manitoba to Iend or sell copies of this thesis/practicum, to the National Library of Canada to microfilm this thesis and to lend or sell copies of the film, and to Dissertations Abstracts International to publish an abstract of this thesis/practicum.

The author reserves other publication rights, and neither this thesis/practicum nor extensive extracts from it may be printed or otherwise reproduced without the author's written permission. 


\begin{abstract}
Many common structural members can be thought of as an assemblage of thin plates. For example, an I shaped cross-section can be made from three or five thin plates and an angle can be thought of as two thin plates whose sides are rigidly attached at an angle, and so on. In this study a multi-purpose computer program was developed, based on a Rayleigh-Ritz (RR) type stiffness approximation, to investigate the wave propagation in infinitely long thin-walled members and the free vibration of thin-walled members that are simply supported at their ends. Also, wave propagation characteristics of these members where studied. To model the behaviour of these types of structural members, using a finite element methodology, an element that closely models the behaviour of a thin plate was created. There are two uncoupled motions of a homogeneous thin plate having material symmetry about its middle surface; one corresponds to inplane motion and the other to bending. Previous studies used a three node parabolic element to model the inplane motion of the plate. In the present work, the three node inplane element and a two node beam element were used to generate an element which models both the inplane and bending motions of a thin plate. The program was checked for accuracy against another approximate solutions as well as analytical solutions. The Rayleigh-Ritz approximation proved to be effective in calculating the wave dispersion characteristics (wavenumber and modeshapes for a given frequency) of thin-walled, infinitely long members as well as the characteristic frequencies of vibration of simply supported thin-walled structural members.
\end{abstract}




\section{ACKNOWLEDGMENTS}

Thanks

- to my brother, parents and grandparents who have helped me throughout my university education.

- to Colleen and Wayne Flather for making university life less difficult.

- to Jennifer Moore for making life more interesting.

- to Dr. A.H. Shah for serving as my advisor and for providing his experience, support and guidance.

- to Dr. N. Popplewell and Dr. J. Frye for serving as examiners.

- to Dr. W. Zhuang, Dr. J. Zhu and numerous others that have also helped in my studies. 


\section{Contents}

$\begin{array}{ll}\text { Acknowledgments } & \text { ii }\end{array}$

List of Tables $\quad$ vi

List of Figures $\quad$ viii

1 Introduction $\quad 1$

1.1 Purpose .............................. 1

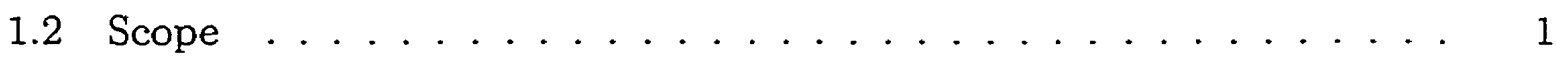

1.3 Overview of the Present Study ................. 2

1.4 Organization of the Thesis ..................... 3

2 Stiffness Method - Two-Dimensional Analysis $\quad 4$

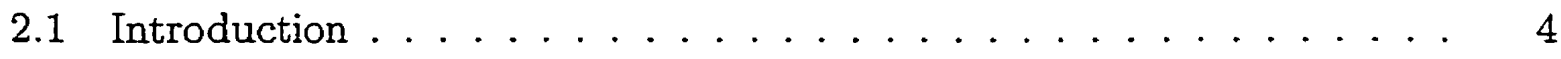

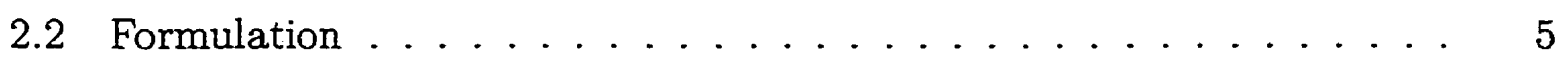

2.3 Eigenvalue Problem ... . . . . . . . . . . . . 16

2.4 Summary ............................... 18 
3 Stiffness Method - Three-Dimensional Analysis

3.1 Introduction . . . . . . . . . . . . . . . 19

3.2 Formulation . . . . . . . . . . . . . . . . 19

3.3 Eigenvalue Problem . . . . . . . . . . . . . . . 25

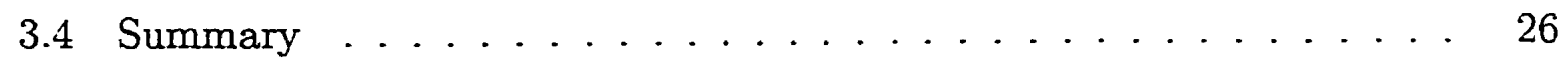

4 Analytical Methods $\quad 27$

4.1 Introduction . . . . . . . . . . . . . . 27

4.2 Analytical Method for Inplane Problem . . . . . . . . . . . 27

4.2 .1 Governing equations $\ldots \ldots \ldots \ldots \ldots \ldots$

4.2 .2 Dispersion Relations . . . . . . . . . . . . . . 29

4.2 .3 Solution . . . . . . . . . . . . . . . . . 30

4.3 Analytical Method for Bending . . . . . . . . . . . 32

4.3 .1 General . . . . . . . . . . . . . . . . 32

4.3.2 Description of the problem . . . . . . . . . 32

4.3.3 Governing equations $\ldots \ldots \ldots \ldots \ldots . \ldots . \ldots . \ldots$

4.3 .4 Solution . . . . . . . . . . . . . . . 34

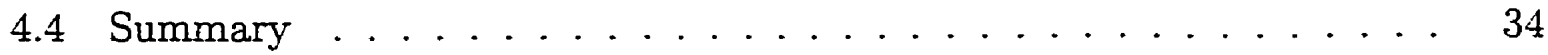

5 Numerical Results $\quad 35$

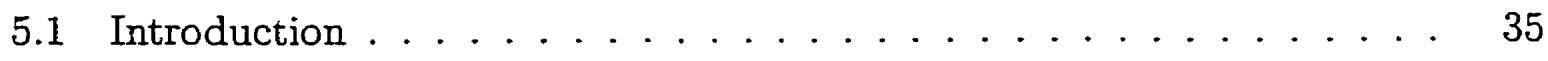

5.2 Wave Propagation Problem $\ldots \ldots \ldots \ldots \ldots$

5.3 The Vibration of Structural Members Simply Supported at Their Ends 40 iv 
5.4 Free Transverse Vibration of Rectangular Plates Simply Supported on Four Edges . . . . . . . . . . . . . . . . 4 47

5.5 Numerical Difficulties at High Frequencies . . . . . . . . . . 49

5.6 Summary . . . . . . . . . . . . . . . . . 49

6 Conclusions $\quad 50$

6.1 Concluding Remarks . . . . . . . . . . . . . . . . 50

6.2 Future Work . . . . . . . . . . . . . . . . . 50

$\begin{array}{ll}\text { References } & 52\end{array}$

A Matrices of the 2D Wave Propagation Problem 54

B 2D RR Vibration of Simply Supported Members 60

B.1 Introduction . . . . . . . . . . . . . . 60

B.2 Formulation . . . . . . . . . . . . . . 60

B.3 Eigenvalue Problem . . . . . . . . . . . . . . . 63

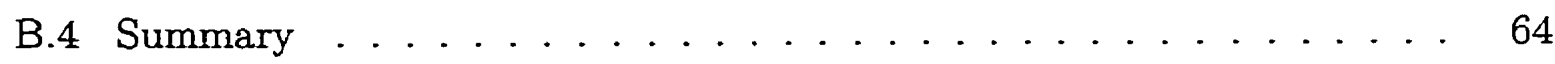

C Matrices for 2D RR Vibration Problem 65

D 3D RR Vibration of Simply Supported Members 72

D.1 Introduction . . . . . . . . . . . . . . . 72

D.2 Formulation ............................. 72

D.3 Eigenvalue Problem . . . . . . . . . . . . . . . . 75

D.4 Summary . . . . . . . . . . . . . . 75 
E Coefficients of Matrix $[P]$ 


\section{List of Tables}

5.1 A comparison of the two-dimensional stiffness approximation and inplane analytical method for calculating circular frequencies of wave propagation, $\mathrm{rad} / \mathrm{s}$, in a plate. . . . . . . . . . 36

5.2 A comparison of the two-dimensional stiffness approximation and bending analytical method for calculating circular frequencies of wave propagation, $\mathrm{rad} / \mathrm{s}$, in a plate. . . . . . . . . . . .

5.3 A comparison of the 2D stiffness approximation and 3D stiffness approximation for calculating circular frequencies of wave propagation, $\mathrm{rad} / \mathrm{s}$, in a plate. . . . . . . . . . . . . . . .

5.4 A comparison of the 2D stress stiffness approximation, 3D stiffness approximation and analytical solution by showing computed circular frequencies of wave propagation, $\omega \mathrm{rad} / \mathrm{s}$, in a cylinder. . . . . . .

5.5 The circular frequencies of vibration, $\mathrm{rad} / \mathrm{s}$, for a simply supported plate calculated using two-dimensional stiffness method.

5.6 The free longitudinal circular frequencies of vibration, $\mathrm{rad} / \mathrm{s}$, of a simply supported plate calulated using the simplified solution. . . . . . . 
5.7 The free, out of plane, transverse circular frequencies of vibration, $\mathrm{rad} / \mathrm{s}$, of a simply supported plate calculated using the simplified soIution. . . . . . . . . . . . . . . . . . .

5.8 The free inplane transverse circular frequencies of vibration, $\mathrm{rad} / \mathrm{s}$, of a simply supported plate calculated using the simplified solution. . .

5.9 A comparison of the 2D stiffness approximation, 3D stiffness approximation and analytical solution by showing computed circular frequencies of free vibration in the $\mathrm{X}-\mathrm{Z}$ plane of an I shaped member, simply supported at its ends. . . . . . . . . . . . . . .

5.10 A comparison of the 2D stiffness approximation, 3D stiffness approximation and analytical solution by showing computed circular frequencies of free vibration in the X-Y plane of an I shaped member, simply supported at its ends. . . . . . . . . . . . . . . .

5.11 The free, out of plane, transverse circular frequencies of vibration, $\mathrm{rad} / \mathrm{s}$, of a rectangular plate, simply supported along all four edges, calculated using the simplified solution. $\ldots \ldots \ldots \ldots \ldots$

5.12 The circular frequencies of vibration, $\mathrm{rad} / \mathrm{s}$, of a plate, simply supported along all four edges, calculated using the two-dimensional stiffness method. . . . . . . . . . . . . . . . . 


\section{List of Figures}

2.1 The geometry of a plate in the local coordinate system. . . . . . 5

2.2 The inplane and bending elements. . . . . . . . . . . 6

2.3 Sign conventions for inplane forces. . . . . . . . . . . 7

2.4 Sign convention for bending moments and shear forces. . . . . . . 11

2.5 The geometry of a single element in the global coordinate system. . . 15

4.1 The geometry of a plate in the local coordinate system. . . . . . 2 28

4.2 The geometry of a plate. . . . . . . . . . . . 33

5.1 The geometry of the I shaped section in the global coordinate system. 44

5.2 A sketch of the mode shape corresponding to $\omega_{1 y y} \ldots \ldots \ldots 46$

5.3 A sketch of the mode shape corresponding to $\omega_{1 z z} \ldots \ldots \ldots 46$ 


\section{Chapter 1}

\section{Introduction}

\subsection{Purpose}

The purpose of this study is to create a multi-purpose computer program that models vibration and wave propagation in thin-walled structural members using a plane stress, Rayleigh-Ritz stiffness aproximation.

\subsection{Scope}

A computer program is developed in this study which is used to investigate wave propagation in infinitely long members as well as the free vibration of simply supported beams. The study will include a description of the Rayleigh-Ritz approximation using two-dimensional theory. As well, exact analytical solutions and a Rayleigh-Ritz approximation based on three-dimensional theory will also be developed for the purpose of verifying the numerical results of the two-dimensional Rayleigh-Ritz approximation. 


\subsection{Overview of the Present Study}

Guided ultrasonic waves have been considered recently for use in the nondestructive testing of materials to detect and characterize flaws that are created during the process of fabrication and flaws that develop throughout the service life of the product. To evaluate the effectiveness of any ultrasonic nondestructive evaluation method it is important to understand the behaviour of the propagating waves as they travel through a member in order. One section of this study focuses on the analysis of guided waves in thin-walled structural members. Specifically, it studies the wave dispersion characteristics (wavenumber and modeshapes for a given frequency) of the member. This information is necessary for the quantitative nondestructive evaluation of the member. The inspiration for this study came from sucesses in modeling the wave propagation in plates of infinite length and width using a Rayleigh-Ritz approximation for plane strain analysis [1]-[6]. Although the plane strain stiffness approximation does reasonably model the behaviour of an infinitely long and wide plate it was recognized that the majority of structural members in use today cannot be regarded as being both infinitely long and wide. The majority of structural members, such as I-shapes or C-shapes, fall into a group that can be thought of as an assemblage of thin plates. In this study a two-dimensional Rayleigh-Ritz approximation will be used to calculate the wave dispersion characteristics of thin-walled structural members assuming that plane stress conditions are satisfied.

Also included in this report is a new look at calculating the characteristic frequencies of vibration of members that are simply supported at two ends. Previous methods of calculating the characteristic frequencies of vibration of simply supported members have assumed that the cross-section geometry of the section remains constant throughout each cycle of vibration. The Rayleigh-Ritz method described in this report accounts for the distortion of the cross-sectional geometry during vibration. 


\subsection{Organization of the Thesis}

Chapter 2 consists of the formulation of the two-dimensional Rayleigh-Ritz stiffness approximation for wave propagation in thin-walled structural members. This approximation is an extension of the plane strain stiffness approximation that was described earlier in the Chapter. The next chapter, Chapter 3, formulates the same wave propagation problem using three-dimensional theory instead of two-dimensional theory. This formulation has appeared in previous papers by Dong and Kazic [7] and it will be used to check the accuracy of the two-dimensional Rayleigh-Ritz stiffness approximation formulated in Chapter 2. In Chapter 4, the formulation of the exact analytical solution for both the inplane and bending motions of a thin-walled structural member is presented. The theory for the analytical solution of the inplane wave propagation problem was created by modifying the plane strain solution presented by Karu [8] and Zhu [9]. Chapter 5 includes all the numerical results needed to verify the accuracy of the plane stress stiffness approximation. Finally, Chapter 6 will conclude with the findings as well as suggest future work that can be done using the theory and programs resulting from this study. 


\section{Chapter 2}

\section{Stiffness Method -}

\section{Two-Dimensional Analysis}

\subsection{Introduction}

Many structural members can be thought of as an assemblage of thin plates. For example an I shaped cross-section can be made from three or five thin plates and an angle can be thought of as two thin plates whose sides are attached at an angle, and so on. Thus, to model these types of structural members using the finite element methodology we must first create an element that closely models a thin plate. There are two uncoupled motions of a homogeneous thin plate having material symmetry about its middle surface; inplane and bending. In this chapter a stiffness method, wherein the displacements are approximated by the interpolation of discrete nodal (interface) values, is presented in order to study the wave propagation in structural members of infinite length in the longitudinal direction. Previous studies have used a three node parabolic element to model the inplane motion of the plane strain problem and has been proven to be reliable when compared to analytical solutions [1]-[6]. In 
the present work, the three node inplane element and a two node bending element are assembled to generate an element that models both the inplane and bending motions of a thin plate.

\subsection{Formulation}

Figure 2.1 shows the geometry of a thin plate in the local $x, y, z$ coordinate system. This is the thin plate from which the element stiffness equations will be derived using standard finite element methodology. The plate has infinite length or a simply supported length, $a$, in the local $\mathrm{x}$ direction, a height, $L$, in the local $y$ direction and a thickness, $h$, in the local $z$ direction such that $h / L<10$.

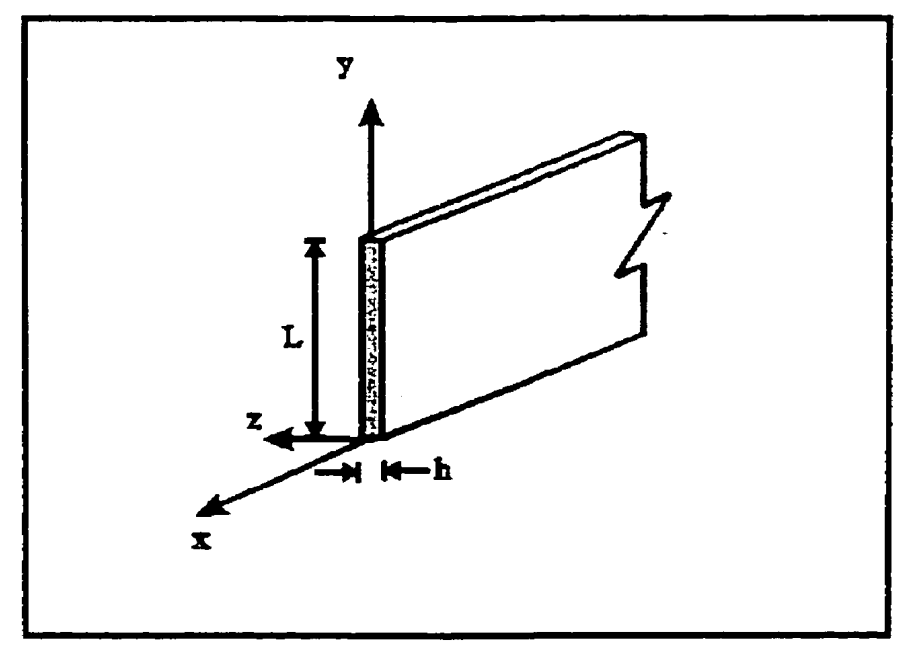

Figure 2.1: The geometry of a plate in the local coordinate system.

The three node inplane element and the two node bending elements are shown in Figure 2.2. In this formulation, the equations for the inplane element and the two beam elements are derived separately. The equations are then assembled to generate a three node element capable of representing inplane and bending motions. 


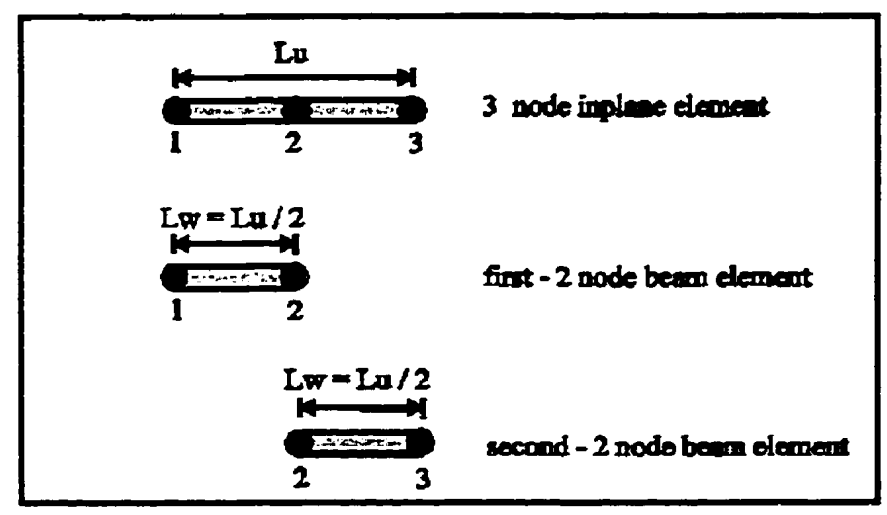

Figure 2.2: The inplane and bending elements.

For a straight plate the inplane and bending motions are uncoupled. However, when more than one plate is assembled to form common structural shapes the two motions become coupled.

The Rayleigh-Ritz stiffness approximation uses Hamilton's energy principle to generate the elemental equations:

$$
\delta \int_{t_{1}}^{t_{2}}(T-\Pi) d t=0
$$

where $T$ and $\Pi$ are the kinetic and potential energies in the body. The expression for the kinetic energy resulting from the inplane motions of an element is given by:

$$
T=\frac{1}{2} \iint \rho\{\dot{u}\}^{T}\{\dot{u}\} d y d x
$$

where $\rho$ is the mass density per unit of area, $\{u\}^{T}=\langle u v\rangle$ is a displacement array and a dot indicates differentiation with respect to time.

The potential energy, $\Pi$, for the inplane motion is given by:

$$
\Pi=\frac{1}{2} \iint\{\varepsilon\}^{T}\left[D^{u}\right]\{\varepsilon\} d y d x
$$

where $\{\varepsilon\}$ is an array of strain. In Equation 2.3, the work done by the boundary force, $\left\{F_{u}\right\}$, is omitted for convenience. 
The strain-displacement relationships for each element are

$$
\{\varepsilon\}=\left\{\begin{array}{c}
\varepsilon_{x x} \\
\varepsilon_{y y} \\
\gamma_{x y}
\end{array}\right\}=\left\{\begin{array}{c}
\frac{\partial u}{\partial x} \\
\frac{\partial v}{\partial y} \\
\frac{\partial v}{\partial x}+\frac{\partial u}{\partial y}
\end{array}\right\}
$$

where $u$ and $v$ are the displacements in the $x$ and $y$ directions respectively. $\varepsilon_{x x}, \varepsilon_{y y}$ and $\gamma_{x y}$ are the inplane strains.

The stress-strain relationships are

$$
\{N\}=\left[D^{u}\right]\{\varepsilon\} .
$$

In Equation 2.5 the inplane forces are $\{N\}=\left\{N_{x x}, N_{y y}, N_{x y}\right\}^{T}$. The sign conventions for the inplane forces are shown in Figure 2.3.

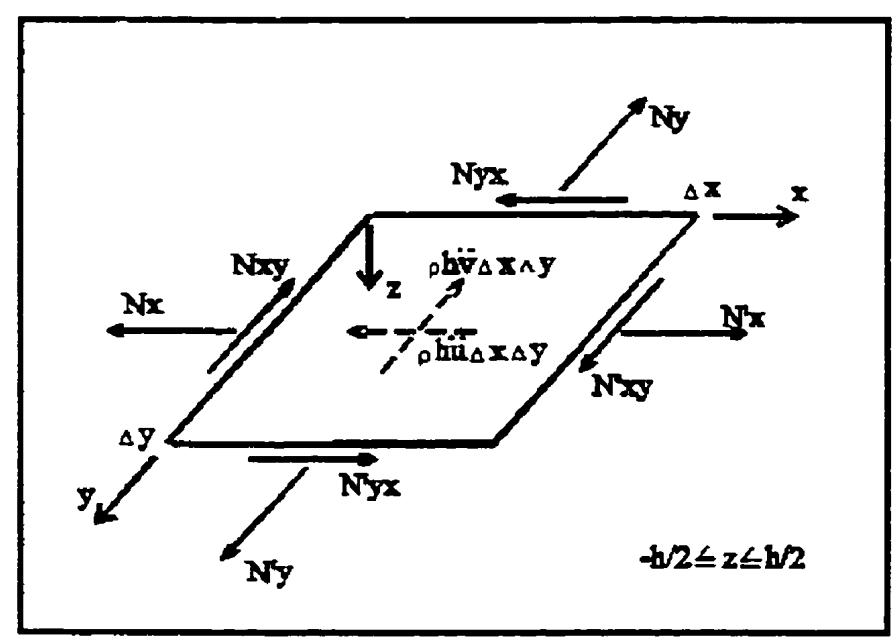

Figure 2.3: Sign conventions for inplane forces.

Here generalized plane stress conditions are assumed as

$$
\left[D^{u}\right]=h\left[\begin{array}{ccc}
D_{11} & D_{12} & 0 \\
D_{21} & D_{22} & 0 \\
0 & 0 & D_{66}
\end{array}\right]
$$


where $D_{11}=\frac{E_{x}}{1-\nu_{x y} \nu_{y x}}, D_{22}=\frac{E_{y}}{1-\nu_{x y} \nu_{y x}}, D_{12}=D_{21}=\frac{\nu_{x y} E_{y}}{1-\nu_{x y} \nu_{y x}}, D_{66}=\frac{E_{x}\left(1-\nu_{x y}\right)}{2\left(1-\nu_{x y} \nu_{y x}\right)} \cdot E_{x}$ and $E_{y}$ are the modulus of elasticity in the $\mathrm{x}$ and $\mathrm{y}$ directions respectively, $\nu$ is defined as Poisson's ratio.

The nodal displacements are approximated as:

$$
\{u\}=\left\{\begin{array}{l}
u(x, y, t) \\
v(x, y, t)
\end{array}\right\}=\left[N^{u}(y)\right]\{q(x, t)\}=\left[N^{u}\right]\{q\}
$$

for the inplane element, where

$$
\left[N^{u}(y)\right]=\left[\begin{array}{cccccccccccc}
N_{1}^{u} & 0 & 0 & 0 & N_{2}^{u} & 0 & 0 & 0 & N_{3}^{u} & 0 & 0 & 0 \\
0 & N_{1}^{u} & 0 & 0 & 0 & N_{2}^{u} & 0 & 0 & 0 & N_{3}^{u} & 0 & 0
\end{array}\right]
$$

and

$$
\{q(x, t)\}=\left\{\begin{array}{c}
u_{1}(x, t) \\
v_{1}(x, t) \\
w_{1}(x, t) \\
\theta_{1}(x, t) \\
u_{2}(x, t) \\
v_{2}(x, t) \\
w_{2}(x, t) \\
\theta_{2}(x, t) \\
u_{3}(x, t) \\
v_{3}(x, t) \\
w_{3}(x, t) \\
\theta_{3}(x, t)
\end{array}\right\}
$$

where $u_{i}, v_{i}, w_{i}$ are the nodal displacements in the $x, y, z$ direction and $\theta_{i}$ is the rotation about the $z$ axis at node i.

Shape functions used for the inplane element are

$$
N_{1}^{u}=\frac{1}{2}\left(\eta^{2}-\eta\right), N_{2}^{u}=1-\eta^{2}, N_{3}^{u}=\frac{1}{2}\left(\eta^{2}+\eta\right)
$$

where $0 \leq y \leq L_{u}$ and $y=\frac{L_{u}}{2} \eta+\frac{L_{u}}{2}$.

The strains in Equation 2.4 can be written in terms of the shape functions $[N(y)]$ and displacement array $\{q(x, t)\}$. For the inplane element

$$
\{\varepsilon\}=[A]\left\{q^{\prime}\right\}+[B]\{q\}
$$


where

$$
[A]=\left[\begin{array}{cccccccccccc}
N_{1}^{u} & 0 & 0 & 0 & N_{2}^{u} & 0 & 0 & 0 & N_{3}^{u} & 0 & 0 & 0 \\
0 & 0 & 0 & 0 & 0 & 0 & 0 & 0 & 0 & 0 & 0 & 0 \\
0 & N_{1}^{u} & 0 & 0 & 0 & N_{2}^{u} & 0 & 0 & 0 & N_{3}^{u} & 0 & 0
\end{array}\right]
$$

and

$$
[B]=\left[\begin{array}{cccccccccccc}
0 & 0 & 0 & 0 & 0 & 0 & 0 & 0 & 0 & 0 & 0 & 0 \\
0 & \frac{\partial N_{1}^{u}}{\partial y} & 0 & 0 & 0 & \frac{\partial N_{2}^{u}}{\partial y} & 0 & 0 & 0 & \frac{\partial N_{3}^{u}}{\partial y} & 0 & 0 \\
\frac{\partial N_{1}^{u}}{\partial y} & 0 & 0 & 0 & \frac{\partial N_{2}^{u}}{\partial y} & 0 & 0 & 0 & \frac{\partial N_{3}^{u}}{\partial y} & 0 & 0 & 0
\end{array}\right]
$$

Substituting Equation 2.7 into the equation for kinetic energy, Equation 2.2, results in:

$$
T=\frac{1}{2} \iint \rho\{\dot{q}\}^{T}\left(\left[N^{u}\right]^{T}\left[N^{u}\right]\right)\{\dot{q}\} d y d x
$$

In a similar manner, substituting Equation 2.9 into the equation for potential energy, Equation 2.3, gives:

$$
\begin{aligned}
\Pi & =\frac{1}{2} \iint\left[\{q\}^{T}\left([A]^{T}\left[D^{u}\right][A]\right)\{q\}+\{q\}^{T}\left([A]^{T}\left[D^{u}\right][B]\right)\{q\}^{\prime}\right. \\
& \left.+\{q\}^{\prime T}\left([B]^{T}\left[D^{u}\right][A]\right)\{q\}+\{q\}^{\prime T}\left([B]^{T}\left[D^{u}\right][B]\right)\{q\}^{\prime}\right] d y d x
\end{aligned}
$$

Application of Hamilton's principle, Equation 2.1, gives:

$$
\left[K_{1}\right]\left\{q^{\prime \prime}\right\}+\left[K_{2}\right]\left\{q^{\prime}\right\}-\left[K_{3}\right]\{q\}-[M]\{\ddot{q}\}=0
$$

where

$$
\begin{aligned}
& {\left[K_{1}\right]=\iint[B]^{T}\left[D^{u}\right][B] d y} \\
& {\left[K_{2}\right]=\iint\left([B]^{T}\left[D^{u}\right][A]-[A]^{T}\left[D^{u}\right][B]\right) d y} \\
& {\left[K_{3}\right]=\iint[A]^{T}\left[D^{u}\right][A] d y} \\
& {[M]=\iint \rho[N]^{T}[N] d y .}
\end{aligned}
$$

Note that $\left[K_{1}\right],\left[K_{3}\right]$ and $[M]$ are symmetric while $\left[K_{2}\right]$ is antisymmetric. The elements of $[M],\left[K_{1}\right],\left[K_{2}\right]$, and $\left[K_{3}\right]$ of Equation 2.14 are defined in Appendix A. In Equation 
2.14 the overdot and prime denote the differentiation with respect to time and $x$ respectively.

The element equations for the two bending elements are created by employing a similar methodology. For the bending problem the kinetic energy is given by:

$$
T=\frac{1}{2} \iint \rho \dot{w}^{2} d y d x
$$

where $\rho$ is the mass density per unit of area, $w$ is a displacement in the z-direction and a dot again indicates differentiation with respect to time.

The potential energy for bending is given by:

$$
\left.\Pi=\iint \frac{1}{2}\{k\}^{T}\left[D^{w}\right]\{k\}\right] d y d x
$$

where $\{k\}$ is an array of curvatures. The work done by the external forces, $\left\{F_{w}\right\}$, has been omitted for convenience.

The curvature-displacement relationship for the bending elements are

$$
\{k\}=\left\{\begin{array}{c}
k_{x x} \\
k_{y y} \\
k_{x y}
\end{array}\right\}=\left\{\begin{array}{c}
-\frac{\partial^{2} w}{\partial x^{2}} \\
-\frac{\partial^{2} w}{\partial y^{2}} \\
-2 \frac{\partial^{2} w}{\partial x \partial y}
\end{array}\right\}
$$

where $w$ is the displacement in the $z$ direction and $k_{x x}, k_{y y}$ and $k_{x y}$ are the curvatures.

The moment-curvature relationship is:

$$
\{M\}=\left[D^{w}\right]\{\kappa\} .
$$

In Equations 2.22 the bending moments are $\{M\}=\left\{M_{x x}, M_{y y}, M_{x y}\right\}^{T}$. The sign conventions for the bending moments are shown in Figure 2.4. Here generalized plane stress conditions are assumed and, therefore, $\left[D^{w}\right]=\frac{h^{2}}{12}\left[D^{u}\right]$ where $\left[D^{u}\right]$ was defined earlier in Equation 2.6.

The nodal displacements are approximated as:

$$
w_{1}(x, y, t)=\left[N^{w_{1}}(y)\right]\{q(x, t)\}
$$




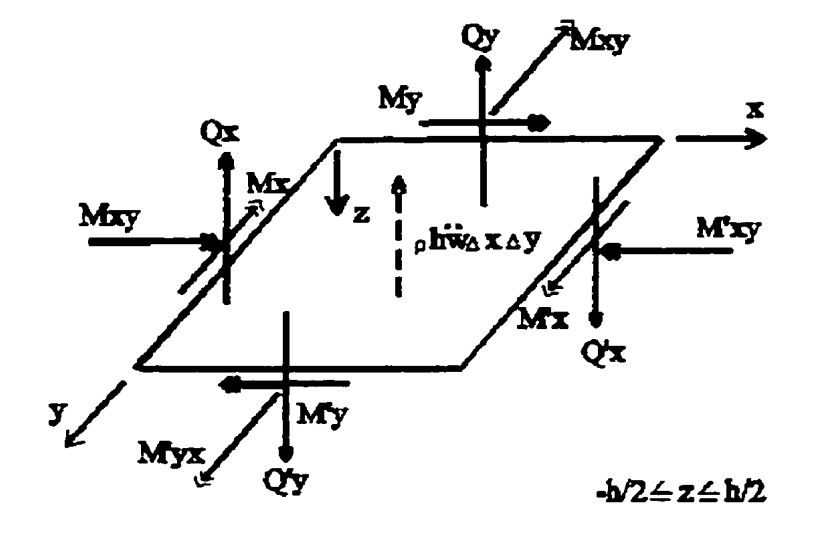

Figure 2.4: Sign convention for bending moments and shear forces.

for the first bending element, and

$$
w_{2}(x, y, t)=\left[N^{w_{2}}(y)\right]\{q(x, t)\}
$$

for the second bending element. $\left[N^{w 1}(y)\right]$ and $\left[N^{w 2}(y)\right]$ are matrices containing the shape functions defined below:

$$
\begin{aligned}
& {\left[N^{w_{1}}(y)\right]=\left[\begin{array}{llllllllllll}
0 & 0 & N_{1}^{w} & N_{2}^{w} & 0 & 0 & N_{3}^{w} & N_{4}^{w} & 0 & 0 & 0 & 0
\end{array}\right]} \\
& {\left[N^{w_{2}}(y)\right]=\left[\begin{array}{llllllllllll}
0 & 0 & 0 & 0 & 0 & 0 & N_{1}^{w} & N_{2}^{w} & 0 & 0 & N_{3}^{w} & N_{4}^{w}
\end{array}\right]}
\end{aligned}
$$

For both the bending elements, the shape functions are

$$
\begin{gathered}
N_{1}^{w}=\frac{1}{4}\left(2-3 \eta+\eta^{3}\right), N_{2}^{w}=\frac{L_{w}}{8}\left(1-\eta-\eta^{2}+\eta^{3}\right) \\
N_{3}^{w}=\frac{1}{4}\left(2+3 \eta-\eta^{3}\right), N_{4}^{w}=\frac{L_{w}}{8}\left(-1-\eta+\eta^{2}+\eta^{3}\right)
\end{gathered}
$$

where $0 \leq y \leq L_{w}, y=\frac{L_{w}}{2} \eta+\frac{L_{w}}{2}$ and $L_{w}=\frac{L_{u}}{2}$.

The curvatures in Equation 2.21 can be rewritten now in terms of the shape functions $[N(y)]$ and the displacement array $\{q(x, t)\}$. For each beam element 


$$
-\{k\}=\left[d_{\alpha}\right]\left\{q^{\prime \prime}\right\}+\left[b_{\alpha}\right]\left\{q^{\prime}\right\}+\left[a_{\alpha}\right]\{q\}
$$

where $\alpha=1$ or 2 . Thus for beam element one $(\alpha=1)$, matrices $\left[d_{1}\right],\left[b_{1}\right]$ and $\left[a_{1}\right]$ are defined as:

$$
\begin{gathered}
{\left[d_{1}\right]=\left[\begin{array}{cccccccccccc}
0 & 0 & N_{1}^{w} & N_{2}^{w} & 0 & 0 & N_{3}^{w} & N_{4}^{w} & 0 & 0 & 0 & 0 \\
0 & 0 & 0 & 0 & 0 & 0 & 0 & 0 & 0 & 0 & 0 & 0 \\
0 & 0 & 0 & 0 & 0 & 0 & 0 & 0 & 0 & 0 & 0 & 0
\end{array}\right]} \\
{\left[b_{1}\right]=\left[\begin{array}{cccccccccccc}
0 & 0 & 0 & 0 & 0 & 0 & 0 & 0 & 0 & 0 & 0 & 0 \\
0 & 0 & 0 & 0 & 0 & 0 & 0 & 0 & 0 & 0 & 0 & 0 \\
0 & 0 & 2 \frac{\partial N_{1}^{w}}{\partial y} & 2 \frac{\partial N_{2}^{w}}{\partial y} & 0 & 0 & 2 \frac{\partial N_{3}^{w}}{\partial y} & 2 \frac{\partial N_{4}^{w}}{\partial y} & 0 & 0 & 0 & 0
\end{array}\right]} \\
{\left[a_{1}\right]=\left[\begin{array}{cccccccccccc}
0 & 0 & 0 & 0 & 0 & 0 & 0 & 0 & 0 & 0 & 0 & 0 \\
0 & 0 & \frac{\partial^{2} N_{1}^{w}}{\partial y^{2}} & \frac{\partial^{2} N_{2}^{w}}{\partial y^{2}} & 0 & 0 & \frac{\partial^{2} N_{3}^{w}}{\partial y^{2}} & \frac{\partial^{2} N_{4}^{w}}{\partial y^{2}} & 0 & 0 & 0 & 0 \\
0 & 0 & 0 & 0 & 0 & 0 & 0 & 0 & 0 & 0 & 0 & 0
\end{array}\right]}
\end{gathered}
$$

and, for beam element two $(\alpha=2)$, matrices $\left[d_{2}\right],\left[b_{2}\right]$ and $\left[a_{2}\right]$ are defined as:

$$
\begin{gathered}
{\left[d_{2}\right]=\left[\begin{array}{cccccccccccc}
0 & 0 & 0 & 0 & 0 & 0 & N_{1}^{w} & N_{2}^{w} & 0 & 0 & N_{3}^{w} & N_{4}^{w} \\
0 & 0 & 0 & 0 & 0 & 0 & 0 & 0 & 0 & 0 & 0 & 0 \\
0 & 0 & 0 & 0 & 0 & 0 & 0 & 0 & 0 & 0 & 0 & 0
\end{array}\right]} \\
{\left[b_{2}\right]=\left[\begin{array}{cccccccccccc}
0 & 0 & 0 & 0 & 0 & 0 & 0 & 0 & 0 & 0 & 0 & 0 \\
0 & 0 & 0 & 0 & 0 & 0 & 0 & 0 & 0 & 0 & 0 & 0 \\
0 & 0 & 0 & 0 & 0 & 0 & 2 \frac{\partial N_{1}^{w}}{\partial y} & 2 \frac{\partial N_{2}^{w}}{\partial y} & 0 & 0 & 2 \frac{\partial N_{3}^{w}}{\partial y} & 2 \frac{\partial N_{4}^{w}}{\partial y}
\end{array}\right]} \\
{\left[a_{2}\right]=\left[\begin{array}{cccccccccccc}
0 & 0 & 0 & 0 & 0 & 0 & 0 & 0 & 0 & 0 & 0 & 0 \\
0 & 0 & 0 & 0 & 0 & 0 & \frac{\partial^{2} N_{u}^{w}}{\partial y^{2}} & \frac{\partial^{2} N_{2}^{w}}{\partial y^{2}} & 0 & 0 & \frac{\partial^{2} N_{3}^{w}}{\partial y^{2}} & \frac{\partial^{2} N_{4}^{w}}{\partial y^{2}} \\
0 & 0 & 0 & 0 & 0 & 0 & 0 & 0 & 0 & 0 & 0 & 0
\end{array}\right]}
\end{gathered}
$$

where a prime denotes differentiation with respect to $y$.

Substituting Equations 2.23 and 2.24 into the equation for the kinetic energy, Equation 2.19, results in:

$$
T=\frac{1}{2} \iint \rho\{\dot{q}\}^{T}\left(\left[N^{w}\right]^{T}\left[N^{w}\right]\right)\{\dot{q}\} d y d x
$$


In a similar manner, substituting Equation 2.26 into the equation for the potential energy, Equation 2.20, gives:

$$
\begin{aligned}
\Pi & =\frac{1}{2} \iint\left[\left\{q^{\prime \prime}\right\}^{T}\left(\left[d_{\alpha}\right]^{T}\left[D^{w}\right]\left[d_{\alpha}\right]\right)\left\{q^{\prime \prime}\right\}+\left\{q^{\prime \prime}\right\}^{T}\left(\left[d_{\alpha}\right]^{T}\left[D^{w}\right]\left[b_{\alpha}\right]\right)\left\{q^{\prime}\right\}\right. \\
& +\left\{q^{\prime \prime}\right\}^{T}\left(\left[d_{\alpha}\right]^{T}\left[D^{w}\right]\left[a_{\alpha}\right]\right)\{q\}+\left\{q^{\prime}\right\}^{T}\left(\left[b_{\alpha}\right]^{T}\left[D^{w}\right]\left[d_{\alpha}\right]\right)\left\{q^{\prime \prime}\right\}+\left\{q^{\prime}\right\}^{T}\left(\left[b_{\alpha}\right]^{T}\left[D^{w}\right]\left[b_{\alpha}\right]\right)\{q\} \\
& +\left\{q^{\prime}\right\}^{T}\left(\left[b_{\alpha}\right]^{T}\left[D^{w}\right]\left[a_{\alpha}\right]\right)\{q\}+\{q\}^{T}\left(\left[a_{\alpha}\right]^{T}\left[D^{w}\right]\left[d_{\alpha}\right]\right)\left\{q^{\prime \prime}\right\}+\{q\}^{T}\left(\left[a_{\alpha}\right]^{T}\left[D^{w}\right]\left[b_{\alpha}\right]\right)\left\{q^{\prime}\right\} \\
& \left.+\{q\}^{T}\left(\left[a_{\alpha}\right]^{T}\left[D^{w}\right]\left[a_{\alpha}\right]\right)\{q\}\right] d y d x
\end{aligned}
$$

where $\alpha=1$ or 2 .

The application of Hamilton's principle for the two beam elements, Equation 2.1, gives:

$$
-\left[M_{b}^{\alpha}\right]\{\ddot{q}\}-\left[E_{1}^{\alpha}\right]\left\{q^{\prime v}\right\}-\left[E_{2}^{\alpha}\right]\left\{q^{\prime \prime \prime}\right\}-\left[E_{3}^{\alpha}\right]\left\{q^{\prime \prime}\right\}-\left[E_{4}^{\alpha}\right]\left\{q^{\prime}\right\}-\left[E_{5}^{\alpha}\right]\{q\}=\{0\}
$$

where

$$
\begin{aligned}
& {\left[E_{1}^{\alpha}\right]=\int\left[d_{\alpha}\right]^{T}\left[D^{w}\right]\left[d_{\alpha}\right] d y} \\
& {\left[E_{2}^{\alpha}\right]=\int\left(\left[d_{\alpha}\right]^{T}\left[D^{w}\right]\left[b_{\alpha}\right]-\left[b_{\alpha}\right]^{T}\left[D^{w}\right]\left[d_{\alpha}\right]\right) d y=[0]} \\
& {\left[E_{3}^{\alpha}\right]=\int\left(\left[d_{\alpha}\right]^{T}\left[D^{w}\right]\left[a_{\alpha}\right]-\left[b_{\alpha}\right]^{T}\left[D^{w}\right]\left[b_{\alpha}\right]+\left[a_{\alpha}\right]^{T}\left[D^{w}\right]\left[d_{\alpha}\right]\right) d y} \\
& {\left[E_{4}^{\alpha}\right]=\int\left(\left[a_{\alpha}\right]^{T}\left[D^{w}\right]\left[b_{\alpha}\right]-\left[b_{\alpha}\right]^{T}\left[D^{w}\right]\left[a_{\alpha}\right]\right) d y=[0]} \\
& {\left[E_{5}^{\alpha}\right]=\int\left[a_{\alpha}\right]^{T}\left[D^{w}\right]\left[a_{\alpha}\right] d y} \\
& {\left[M_{b}^{\alpha}\right]=\int \rho\left[N^{w_{\alpha}}\right]^{T}\left[N^{w_{\alpha}}\right] d y}
\end{aligned}
$$

Note that the non-zero matrices, $\left[E_{1}^{\alpha}\right],\left[E_{3}^{\alpha}\right],\left[E_{5}^{\alpha}\right]$ and $\left[M_{b}^{\alpha}\right]$ are symmetric. In Equation 2.35 the overdot and prime denote differentiation with respect to time and $x$ respectively.

For the two beam elements, the element equations become:

$$
-\left[M_{b}^{1}\right]\{\ddot{q}\}-\left[E_{1}^{1}\right]\left\{q^{\prime v}\right\}-\left[E_{3}^{1}\right]\left\{q^{\prime \prime}\right\}-\left[E_{5}^{1}\right]\{q\}=\{0\}
$$


and

$$
-\left[M_{b}^{2}\right]\{\ddot{q}\}-\left[E_{1}^{2}\right]\left\{q^{\prime v}\right\}-\left[E_{3}^{2}\right]\left\{q^{\prime \prime}\right\}-\left[E_{5}^{2}\right]\{q\}=\{0\}
$$

Adding the equations for the two beam elements, Equations 2.42 and 2.43, results in the three node element equation

$$
-\left[M_{b}\right]\{\ddot{q}\}-\left[E_{1}\right]\left\{q^{\prime \prime}\right\}-\left[E_{3}\right]\left\{q^{\prime \prime}\right\}-\left[E_{5}\right]\{q\}=\{0\}
$$

where $\left[M_{b}\right]=\left[M_{b}^{1}\right]+\left[M_{b}^{2}\right],\left[E_{1}\right]=\left[E_{1}^{1}\right]+\left[E_{1}^{2}\right],\left[E_{3}\right]=\left[E_{3}^{1}\right]+\left[E_{3}^{2}\right]$ and $\left[E_{5}\right]=\left[E_{5}^{1}\right]+\left[E_{5}^{2}\right]$.

The elements of $\left[M_{b}\right],\left[E_{1}\right],\left[E_{3}\right]$ and $\left[E_{5}\right]$ of Equation 2.44 are defined in Appendix A.

For analyzing the propagation of a harmonic wave travelling in the $x$-direction, of a structural member of infinite length in the longitudinal direction, the displacement field can be described as

$$
\{q\}=\{q(x, t)\}=\left\{Q_{0}\right\} e^{+i \omega t} e^{-\gamma x}
$$

where $\gamma$ is the wavenumber and $\omega$ is the circular frequency. The corresponding formulation for the vibration of a structural member simply supported in the $x$-direction $(x=0, a)$ is given in Appendix $\mathrm{B}$, and the matrices appearing therein are presented in Appendix C.

Substituting Equation 2.45 into Equations 2.14 and 2.44, produces Equations 2.46 and 2.47 respectively;

$$
\left[\gamma^{2} K_{1}-\gamma K_{2}-K_{3}^{*}\right]\left\{Q_{0}\right\}=\{0\}
$$

where $\left[K_{3}^{*}\right]=\left[K_{3}\right]-\omega^{2}[M]$ as well as

$$
\left[-\gamma^{4} E_{1}-\gamma^{2} E_{3}-E_{5}^{*}\right]\left\{Q_{0}\right\}=\{0\}
$$

where $\left[E_{5}^{*}\right]=\left[E_{5}\right]-\omega^{2}\left[M_{b}\right]$. 
Adding Equations 2.46 and 2.47 results in the element equations, Equation 2.48, for a thin plate in the local $\mathrm{x}, \mathrm{y}, \mathrm{z}$ coordinate system

$$
\left[\gamma^{2} K_{1}-\gamma K_{2}-K_{3}^{*}-\gamma^{4} E_{1}-\gamma^{2} E_{3}-E_{5}^{*}\right]\left\{Q_{0}\right\}=\{0\}
$$

The next step is to transform the local element equations into the global coordinate system. The local y-z axes of the plate element, shown in Figure 2.5, make an angle $\theta$ with the global $\mathrm{Y}-\mathrm{Z}$ axes.

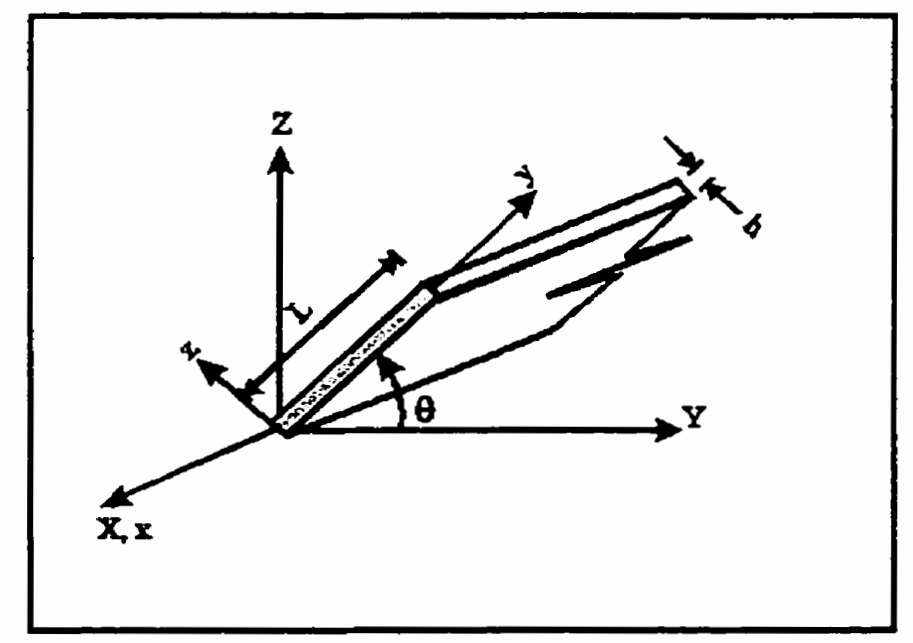

Figure 2.5: The geometry of a single element in the global coordinate system.

The transformation of the element equations from the local to the global coordinate system results in the global element equations, Equation 2.49 , in the form $\left[K_{g}\right]\left\{Q_{0}^{g}\right\}=\{0\}$, i.e.

$$
[T]^{T}\left[\gamma^{2} K_{1}-\gamma K_{2}-K_{3}^{*}-\gamma^{4} E_{1}-\gamma^{2} E_{3}-E_{5}^{*}\right][T]\left\{Q_{0}^{g}\right\}=\{0\}
$$


The elements of $[T]$, in Equation 2.49 are:

$$
[T]=\left[\begin{array}{cccccccccccc}
1 & 0 & 0 & 0 & 0 & 0 & 0 & 0 & 0 & 0 & 0 & 0 \\
0 & C & S & 0 & 0 & 0 & 0 & 0 & 0 & 0 & 0 & 0 \\
0 & -S & C & 0 & 0 & 0 & 0 & 0 & 0 & 0 & 0 & 0 \\
0 & 0 & 0 & 1 & 0 & 0 & 0 & 0 & 0 & 0 & 0 & 0 \\
0 & 0 & 0 & 0 & 1 & 0 & 0 & 0 & 0 & 0 & 0 & 0 \\
0 & 0 & 0 & 0 & 0 & C & S & 0 & 0 & 0 & 0 & 0 \\
0 & 0 & 0 & 0 & 0 & -S & C & 0 & 0 & 0 & 0 & 0 \\
0 & 0 & 0 & 0 & 0 & 0 & 0 & 1 & 0 & 0 & 0 & 0 \\
0 & 0 & 0 & 0 & 0 & 0 & 0 & 0 & 1 & 0 & 0 & 0 \\
0 & 0 & 0 & 0 & 0 & 0 & 0 & 0 & 0 & C & S & 0 \\
0 & 0 & 0 & 0 & 0 & 0 & 0 & 0 & 0 & -S & C & 0 \\
0 & 0 & 0 & 0 & 0 & 0 & 0 & 0 & 0 & 0 & 0 & 1
\end{array}\right]
$$

where $S=\sin (\theta)$ and $C=\cos (\theta)$.

\subsection{Eigenvalue Problem}

The global element equations, Equation 2.49, are assembled to obtain the structure equations, Equation 2.51, in the form $[\mathbf{K}]\left\{\mathbf{Q}_{0}^{\mathbb{g}}\right\}=\{\mathbf{0}\}$, as

$$
\left[\gamma^{2} \mathbf{K}_{1}-\gamma \mathbf{K}_{2}-\mathbf{K}_{3}^{*}-\gamma^{4} \mathbf{E}_{1}-\gamma^{2} \mathbf{E}_{3}-\mathbf{E}_{5}^{*}\right]\left\{\mathbf{Q}_{0}^{\mathrm{g}}\right\}=\{0\}
$$

By defining

$$
\begin{aligned}
& \left\{\mathbf{Q}_{1}^{\mathrm{g}}\right\}=-\gamma\left\{\mathbf{Q}_{0}^{\mathrm{g}}\right\} \\
& \left\{\mathbf{Q}_{2}^{\mathrm{g}}\right\}=-\gamma\left\{\mathbf{Q}_{1}^{\mathrm{g}}\right\} \\
& \left\{\mathbf{Q}_{3}^{\mathrm{g}}\right\}=-\gamma\left\{\mathbf{Q}_{2}^{\mathrm{g}}\right\}
\end{aligned}
$$

and substituting Equations 2.52, 2.53 and 2.54 into Equation 2.51 leads to the eigenvalue problem of the form

$$
[\mathbf{A}]\left\{\mathbf{Q}^{\mathbf{g}}\right\}=\gamma[\mathbf{B}]\left\{\mathbf{Q}^{\mathbf{g}}\right\}
$$


where

$$
\begin{gathered}
\left\{\mathbf{Q}^{\mathrm{g}}\right\}=\left\{\begin{array}{c}
\mathbf{Q}_{0}^{\mathrm{g}} \\
\mathbf{Q}_{1}^{\mathrm{g}} \\
\mathbf{Q}_{2}^{\mathrm{g}} \\
\mathbf{Q}_{3}^{\mathrm{g}}
\end{array}\right\}, \\
{[\mathbf{A}]=\left[\begin{array}{cccc}
0 & -\mathbf{I} & 0 & 0 \\
0 & 0 & -\mathbf{I} & 0 \\
0 & 0 & 0 & -\mathbf{I} \\
-\mathbf{K}_{3}^{*}-\mathbf{E}_{5}^{*} & \mathbf{K}_{2} & -\mathbf{E}_{3} & 0
\end{array}\right]}
\end{gathered}
$$

and

$$
[B]=\left[\begin{array}{cccc}
\mathbf{I} & 0 & 0 & 0 \\
0 & \mathrm{I} & 0 & 0 \\
0 & 0 & \mathrm{I} & 0 \\
0 & \mathrm{~K}_{1} & 0 & -\mathrm{E}_{1}
\end{array}\right]
$$

Solution of the eigenvalue problem determines the wavenumbers, $\gamma$, and the corresponding modeshapes, $\left\{\mathbf{Q}_{0}^{\mathbf{g}}\right\}$, of a member for a given frequency, $\omega$. For physical reasons frequency, $\omega$, is real and positive. The roots for $\gamma$ may be real, complex conjugate pairs or purely imaginary. Positive real roots do not propagate. Complex conjugate pairs with positive real parts and positive imaginary parts represent evanescent modes. These evanescent modes decay in the positive $\mathrm{X}$ direction. Purely positive imaginary roots define waves propagating in the positive $\mathrm{X}$ direction.

Alternatively, Equation 2.51 can be rearranged into the alternative eigenvalue problem, Equation 2.56, where frequencies, $\omega$, are obtained for a given wavenumber, $\gamma$

$$
\left[\gamma^{2} \mathbf{K}_{1}-\gamma \mathbf{K}_{2}-\mathbf{K}_{3}-\gamma^{4} \mathbf{E}_{1}-\gamma^{2} \mathbf{E}_{3}-\mathbf{E}_{5}\right]\left\{\mathbf{Q}_{0}^{\mathrm{g}}\right\}=\omega^{2}\left[-\mathbf{M}-\mathbf{M}_{\mathbf{b}}\right]\left\{\mathbf{Q}_{0}^{\mathrm{g}}\right\}
$$




\subsection{Summary}

The numerical accuracy of the Rayliegh-Ritz type approximation, Equations 2.55 and 2.56 will be discussed in detail in upcoming chapters. The two-dimensional Rayleigh-Ritz approximation will be compared to a similar three-dimensional Rayliegh-Ritz approximation and analytical solutions. These solutions will be presented later in this study. 


\section{Chapter 3}

\section{Stiffness Method -}

\section{Three-Dimensional Analysis}

\subsection{Introduction}

The three-dimensional Rayleigh-Ritz approximation is used to study wave propagation in structural members which are infinite in the longitudinal direction or simply supported at their ends. The three-dimensional analysis presented here will be used later in the study to check the two-dimensional approximation presented earlier.

\subsection{Formulation}

The formulation used for the three-dimensional analysis is similar to that encountered earlier with the plane stress assumption. The Rayleigh-Ritz approximation used in this approach is based on Hamilton's energy principle,

$$
\delta \int_{t_{1}}^{t_{2}}(T-\Pi) d t=0
$$


where $T$ and $\Pi$ are the kinetic and potential energies of the body.

The kinetic energy is given by:

$$
T=\frac{1}{2} \iiint \rho\{\dot{u}\}^{T}\{\dot{u}\} d y d z d x
$$

where $\rho$ is the mass density per unit of volume, $\{u\}$ is a displacement array and a dot indicates differentiation with respect to time. The displacement array $\{u\}$ is given by:

$$
\{u\}^{T}=[u(x, y, z, t) \quad v(x, y, z, t) \quad w(x, y, z, t)] .
$$

The potential energy $\Pi$ is given by

$$
\Pi=\frac{1}{2} \iiint\{\varepsilon\}^{T}[C]\{\varepsilon\} d y d z d x
$$

where the work done by the external forces, $\{F\}$, has been omitted for convenience and $\{\varepsilon\}$ is an array of strains whose components are

$$
\{\varepsilon\}^{T}=\left[\begin{array}{llllll}
\varepsilon_{x x} & \varepsilon_{y y} & \varepsilon_{z z} & \gamma_{y z} & \gamma_{x z} & \gamma_{x y}
\end{array}\right]
$$

A linear strain-displacement relationship is used. Thus the strains can be written as

$$
\{\varepsilon\}=\left\{\begin{array}{c}
\varepsilon_{x x} \\
\varepsilon_{y y} \\
\varepsilon_{z z} \\
\gamma_{y z} \\
\gamma_{x z} \\
\gamma_{x y}
\end{array}\right\}=\left\{\begin{array}{c}
\frac{\partial u}{\partial x} \\
\frac{\partial v}{\partial y} \\
\frac{\partial w}{\partial z} \\
\frac{\partial v}{\partial z}+\frac{\partial w}{\partial y} \\
\frac{\partial u}{\partial z}+\frac{\partial w}{\partial x} \\
\frac{\partial u}{\partial y}+\frac{\partial v}{\partial x}
\end{array}\right\}
$$

where $u, v$ and $w$ are the displacements in the $x, y$ and $z$ directions, respectively. Equation 3.6 can be rewritten, in anticipation of the form required for the solution 
of the problem, as:

$$
\{\varepsilon\}=\left\{\begin{array}{c}
\varepsilon_{x x} \\
\varepsilon_{y y} \\
\varepsilon_{z z} \\
\gamma_{y z} \\
\gamma_{x z} \\
\gamma_{x y}
\end{array}\right\}=\left[\begin{array}{ccc}
0 & 0 & 0 \\
0 & \frac{\partial}{\partial y} & 0 \\
0 & 0 & \frac{\partial}{\partial z} \\
0 & \frac{\partial}{\partial z} & \frac{\partial}{\partial y} \\
\frac{\partial}{\partial z} & 0 & 0 \\
\frac{\partial}{\partial y} & 0 & 0
\end{array}\right]\{u\}+\left[\begin{array}{ccc}
\frac{\partial}{\partial x} & 0 & 0 \\
0 & 0 & 0 \\
0 & 0 & 0 \\
0 & 0 & 0 \\
0 & 0 & \frac{\partial}{\partial x} \\
0 & \frac{\partial}{\partial x} & 0
\end{array}\right]\{u\}
$$

or, in abbreviated matrix form,

$$
\{\varepsilon\}=\left[L_{y z}\right]\{u\}+\left[L_{x}\right]\{u\} .
$$

The stress-strain relationship is

$$
\{\sigma\}=[C]\{\varepsilon\}
$$

In Equation 3.9 the stresses are $\{\sigma\}=\left\{\sigma_{x x}, \sigma_{y y}, \sigma_{z z}, \sigma_{y z}, \sigma_{x z}, \sigma_{x y}\right\}^{T}$. Here we assume three-dimensional relationship where matrix $[C]$ represents the elastic moduli of the material. The formulation of the problem assumes that the structure is completely anisotropic. Thus, there are twenty-one independent coefficients in matrix $[C]$, i.e.,

$$
[C]=\left[\begin{array}{cccccc}
C_{11} & C_{12} & C_{13} & C_{14} & C_{15} & C_{16} \\
& C_{22} & C_{23} & C_{24} & C_{25} & C_{26} \\
& & C_{33} & C_{34} & C_{35} & C_{36} \\
& & & C_{44} & C_{45} & C_{46} \\
& & & & C_{55} & C_{56} \\
\text { Symm. } & & & & & C_{66}
\end{array}\right]
$$

However, for the numerical results presented later it is assumed that the structures are homogeneous and isotropic. Thus, matrix $[C]$ reduces to:

$$
[C]=\left[\begin{array}{cccccc}
C_{11} & C_{12} & C_{13} & 0 & 0 & 0 \\
& C_{22} & C_{23} & 0 & 0 & 0 \\
& & C_{33} & 0 & 0 & 0 \\
& & & C_{44} & 0 & 0 \\
& & & & C_{55} & 0 \\
\text { Symm. } & & & & & C_{66}
\end{array}\right]
$$


where $C_{11}=C_{22}=C_{33}=\frac{E(1-\nu)}{(1+\nu)(1-2 \nu)}, C_{12}=C_{13}=C_{23}=\frac{E \nu}{(1+\nu)(1-2 \nu)}, C_{44}=C_{55}=$ $C_{66}=\frac{E}{2(1+\nu)} . E$ is the modulus of elasticity and $\nu$ is Poisson's ratio.

Isoparametric finite element methodology is followed in forming the element matrices. Three to nine variable-number-noded two-dimensional elements are used to model the shape of the cross-section. Since the numerical integration formulas as well as the direct stiffness assembly procedures are standard there is no need to discuss the details here [10].

The displacement field may be written as:

Substituting

$$
\left\{\begin{array}{c}
u(x, y, z, t) \\
v(x, y, z, t) \\
w(x, y, z, t)
\end{array}\right\}=\left[\begin{array}{c}
\sum N_{i}(y, z) \\
\sum N_{i}(y, z) \\
\sum N_{i}(y, z)
\end{array}\right]\left\{\begin{array}{c}
U_{i}(z, t) \\
Y_{i}(z, t) \\
Z_{i}(z, t)
\end{array}\right\} .
$$

$$
\left\{q_{j}\right\}=\left\{\begin{array}{c}
U_{j} \\
V_{j} \\
W_{j}
\end{array}\right\}
$$

where node $j$ is an intermediate node into Equation 3.33 results in

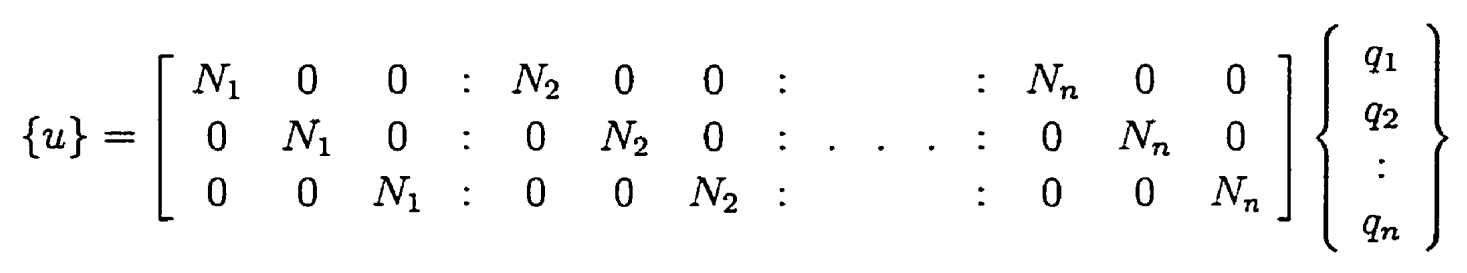

or in abbreviated matrix form

$$
\{u(x, y, z, t)\}=[N(y, z)]\{q(x, t)\}
$$

or simply

$$
\{u\}=[N]\{q\}
$$

Substituting Equation 3.16 into Equation 3.8, we obtain

$$
\{\varepsilon\}=\left[L_{y z}\right][N]\{q\}+\left[L_{x}\right][N]\{q\}
$$


which can be rewritten as,

$$
\{\varepsilon\}=[A]\{q\}+[B]\left\{q^{\prime}\right\}
$$

where

$$
[A]=\left[\begin{array}{ccc}
0 & 0 & 0 \\
0 & N_{, y} & 0 \\
0 & 0 & N_{, z} \\
0 & N_{, z} & N_{, y} \\
N_{, z} & 0 & 0 \\
N_{, y} & 0 & 0
\end{array}\right]
$$

and

$$
[B]=\left[\begin{array}{ccc}
N & 0 & 0 \\
0 & 0 & 0 \\
0 & 0 & 0 \\
0 & 0 & 0 \\
0 & 0 & N \\
0 & N & 0
\end{array}\right]
$$

For the purpose of Guass Quadrature, we consider $6 * 3$ submatrices, $\left[A_{j}\right]$ and $\left[B_{j}\right]$, of the $6 * 3 n$ matrices $[A]$ and $[B]$. Matrix $[A]=\left[L_{y z}\right][N]$, can be rewritten as

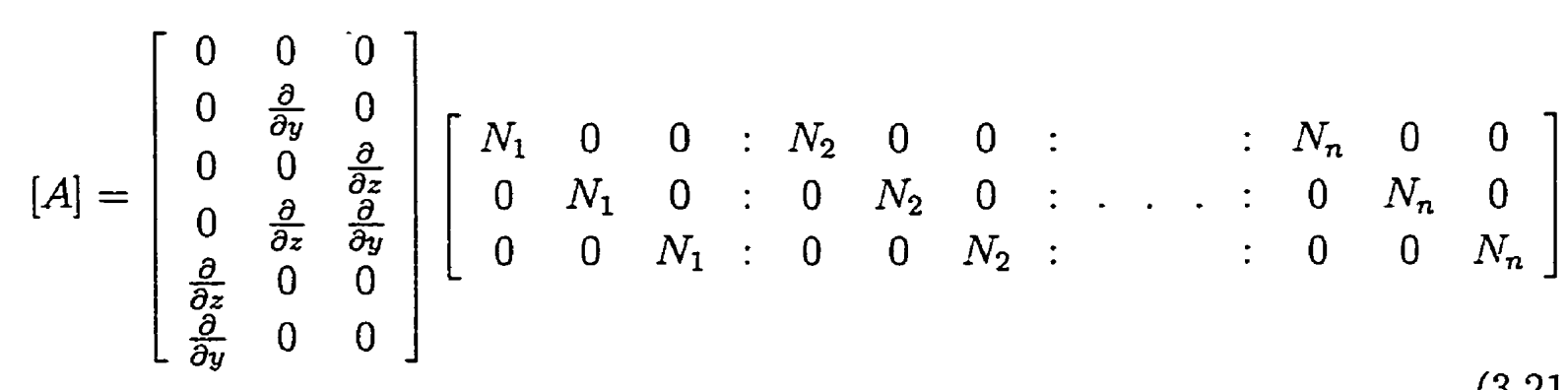

which can also be written as

$$
[A]=\left[\begin{array}{lllllllllll}
{\left[A_{1}\right]} & \mid & {\left[A_{2}\right]} & \mid & \ldots & \mid & {\left[A_{j}\right]} & \mid & \ldots & \mid & {\left[A_{n}\right]}
\end{array}\right] .
$$


Where, for an arbitrary node $j$,

$$
\left[A_{j}\right]=\left[\begin{array}{ccc}
0 & 0 & 0 \\
0 & N_{j, y} & 0 \\
0 & 0 & N_{j, z} \\
0 & N_{j, z} & N_{j, y} \\
N_{j, z} & 0 & 0 \\
N_{j, y} & 0 & 0
\end{array}\right]
$$

and similarly, for matrix $[B]=\left[L_{x}\right][N]$ can be rewritten as

$$
\left[B_{j}\right]=\left[\begin{array}{ccc}
N_{j} & 0 & 0 \\
0 & 0 & 0 \\
0 & 0 & 0 \\
0 & 0 & 0 \\
0 & 0 & N_{j} \\
0 & N_{j} & 0
\end{array}\right]=N_{j}\left[\begin{array}{lll}
1 & 0 & 0 \\
0 & 0 & 0 \\
0 & 0 & 0 \\
0 & 0 & 0 \\
0 & 0 & 1 \\
0 & 1 & 0
\end{array}\right] .
$$

Substituting Equation 3.16 into the expression for the kinetic energy, Equation 3.2, gives:

$$
T=\frac{1}{2} \iiint \rho\{\dot{q}\}^{T}\left([N]^{T}[N]\right)\{\dot{q}\} d y d z d x
$$

In a similar manner, substituting Equation 3.18 into the equation for potential energy, Equation 3.4, gives:

$$
\begin{aligned}
\Pi & =\frac{1}{2} \iiint\left[\{q\}^{T}\left([A]^{T}[C][A]\right)\{q\}+\{q\}^{T}\left([A]^{T}[C][B]\right)\{q\}^{\prime}\right. \\
& \left.+\{q\}^{\prime T}\left([B]^{T}[C][A]\right)\{q\}+\{q\}^{\prime T}\left([B]^{T}[C][B]\right)\{q\}^{\prime}\right] d y d z d x .
\end{aligned}
$$

Where the overdot and prime denote the differentiation with respect to time and $x$ respectively.

Application of Hamilton's principle, Equation 3.1, gives:

$$
\left[K_{1}\right]\left\{q^{\prime \prime}\right\}+\left[K_{2}\right]\left\{q^{\prime}\right\}-\left[K_{3}\right]\{q\}-[M]\{\ddot{q}\}=0
$$


where

$$
\begin{aligned}
{\left[K_{1}\right] } & =\iint[B]^{T}[C][B] d y d z \\
{\left[K_{2}\right] } & =\iint\left[[B]^{T}[C][A]-[A]^{T}[C][B]\right] d y d z \\
{\left[K_{3}\right] } & =\iint[A]^{T}[C][A] d y d z \\
{[M] } & =\iint \rho[N]^{T}[N] d y d z .
\end{aligned}
$$

Note that $\left[K_{1}\right],\left[K_{3}\right]$ and $[M]$ are symmetric while $\left[K_{2}\right]$ is skew-symmetric.

For analyzing the propagation of a harmonic wave travelling in the $x$-direction of a structural member having an infinite length in the longitudinal direction, the displacement field can be described as

$$
\{q(x, t)\}=\left\{Q_{0}\right\} e^{+i \omega t} e^{-\gamma x}
$$

where $\gamma$ is the wavenumber and $\omega$ is the circular frequency. The corresponding formulation for the vibration of a structural member, simply supported in the $x$-direction, $(x=0, a)$ is presented in Appendix D.

Once the elements are assembled by substituting Equation 3.32 into Equation 3.27, one obtains Equation 3.33:

$$
\left[\gamma^{2} \mathbf{K}_{1}-\gamma \mathbf{K}_{2}-\mathbf{K}_{3}+\omega^{2} \mathbf{M}\right]\left\{\mathbf{Q}_{0}\right\}=\{\mathbf{0}\} .
$$

\subsection{Eigenvalue Problem}

By defining

$$
\left\{\mathbf{Q}_{\mathbf{1}}\right\}=-\gamma\left\{\mathbf{Q}_{\mathbf{0}}\right\}
$$

and substituting Equation 3.34 into Equation 3.33, leads to the eigenvalue problem $[\mathbf{A}]\{\mathbf{Q}\}=\gamma[\mathbf{B}]\{\mathbf{Q}\}$ where

$$
\{\mathbf{Q}\}=\left\{\begin{array}{l}
\left\{\mathbf{Q}_{\mathbf{0}}\right\} \\
\left\{\mathbf{Q}_{\mathbf{I}}\right\}
\end{array}\right\}
$$




$$
[A]=\left[\begin{array}{cc}
0 & -\mathbf{I} \\
-\mathbf{K}_{3}+\omega^{2} \mathbf{M} & \mathbf{K}_{2}
\end{array}\right]
$$

and

$$
[\mathrm{B}]=\left[\begin{array}{cc}
\mathrm{I} & 0 \\
0 & \mathrm{~K}_{1}
\end{array}\right] \text {. }
$$

The solution of the eigenvalue problem determines the wavenumber, $\gamma$, and corresponding modeshape, $\left\{\mathbf{Q}_{0}\right\}$, of a member for a given frequency, $\omega$. The roots for $\gamma$ may be real, complex conjugate pairs or purely imaginary. Positive real roots do not propagate. Complex conjugate pairs with positive real parts and positive imaginary parts represent evanescent modes. These evanecent modes decay in the positive $\mathrm{X}$ direction. Purely positive imaginary roots define waves propagating in the positive $\mathrm{X}$ direction.

Alternatively, Equation 3.33 can be arranged into the alternative eigenvalue problem, Equation 3.35, which has the form $[\mathbf{A}]\{\mathbf{Q}\}=\gamma[\mathbf{B}]\{\mathbf{Q}\}$. Frequencies, $\omega$, are obtained for a given wavenumber, $\gamma$, from

$$
\left[\gamma^{2} \mathbf{K}_{1}-\gamma \mathbf{K}_{2}-\mathbf{K}_{\mathbf{3}}\right]\left\{\mathbf{Q}_{\mathbf{0}}\right\}=\omega^{2}[-\mathbf{M}]\left\{\mathbf{Q}_{0}\right\}
$$

\subsection{Summary}

The numerical accuracy of the three-dimensional Rayliegh-Ritz type stiffness approximation, will be discussed in the upcoming chapters. The three-dimensional stiffness approximation will be compared to the two-dimensional Rayliegh-Ritz type stiffness approximation presented earlier and analytical solutions which are presented in the next Chapter. 


\section{Chapter 4}

\section{Analytical Methods}

\subsection{Introduction}

In this chapter the elasticity solutions for inplane and bending problems are presented. The purpose of these formulations is strictly to check the validity of the RayleighRitz procedures, discussed in Chapters 2 and 3, for a thin plate. Thus, the equations derived here are only for the local coordinate system and they are not transformed into the global coordinate system. Moreover, it is not necessary to use the propagator matrices in the inplane problem but it is presented here with the understanding that it may be useful in the future for refining wavenumbers used in a scattering analysis.

\subsection{Analytical Method for Inplane Problem}

In the analytical formulation for the inplane problem, a modified propagator matrix approach is used to establish the frequency equation of the plate. The frequency equation of the plate is formulated in the form of a standard algebraic eigenvalue problem using elasticity equations. 
Consider the plate shown in Figure 4.1. The plate has infinite length in the local $x$ direction, a height, $L$, in the local $y$ direction and a thickness, $h$, in the local $z$ direction.

Plane stress conditions in the $x y$ plane will be considered here.

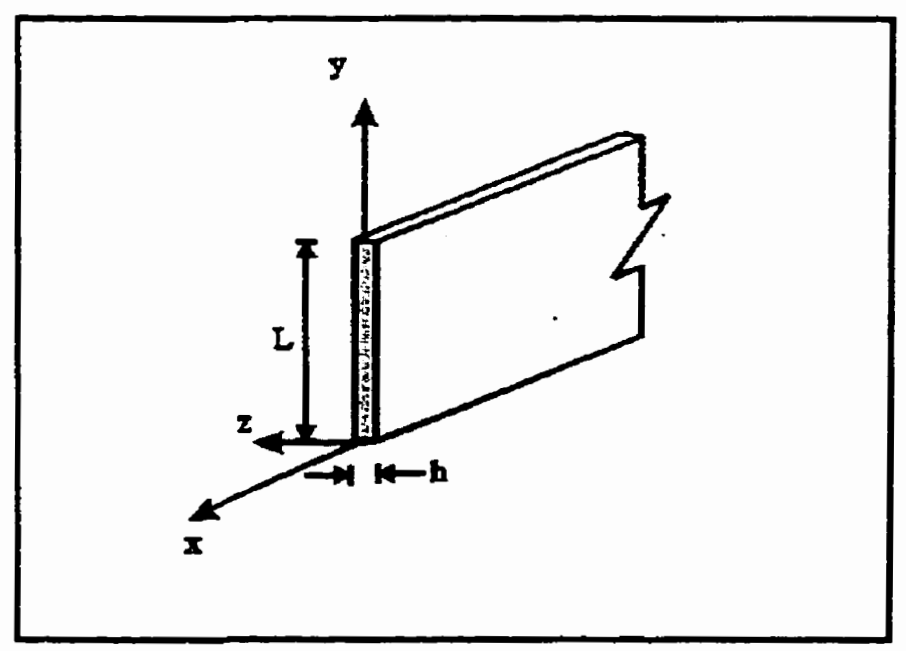

Figure 4.1: The geometry of a plate in the local coordinate system.

\subsubsection{Governing equations}

The strain-displacement relation for the plate is given by

$$
\{\epsilon\}=\mathbf{L u}
$$

where

$$
\mathbf{u}^{T}=\left\{\begin{array}{ll}
u & v
\end{array}\right\} .
$$

The $u$ and $v$ are displacements in $x$ and $y$ directions, respectively, whilst

$$
\mathbf{L}^{T}=\left[\begin{array}{ccc}
\frac{\partial}{\partial x} & 0 & \frac{\partial}{\partial y} \\
0 & \frac{\partial}{\partial y} & \frac{\partial}{\partial x}
\end{array}\right]
$$


and

$$
\{\epsilon\}^{T}=\left\{\begin{array}{lll}
\epsilon_{x x} & \epsilon_{y y} & 2 \epsilon_{y x}
\end{array}\right\}
$$

where $\epsilon_{i j}$ is the strain component. The force-strain relation is given by

$$
\{N\}=\left[D^{u}\right]\{\epsilon\}
$$

where the stress component is defined as

$$
\{\mathbf{N}\}^{\mathrm{T}}=\left\{\begin{array}{lll}
N_{x x} & N_{y y} & N_{y x}
\end{array}\right\} .
$$

Generalized plane stress conditions are assumed. The constitutive matrix for the plate is:

$$
\left[D^{u}\right]=h\left[\begin{array}{ccc}
D_{11} & D_{12} & 0 \\
D_{21} & D_{22} & 0 \\
0 & 0 & D_{66}
\end{array}\right]
$$

where $D_{11}=\frac{E_{x}}{1-\nu_{x y} \nu_{y x}}, D_{22}=\frac{E_{y}}{1-\nu_{x y} \nu_{y x}}, D_{12}=D_{21}=\frac{\nu_{x y} E_{y}}{1-\nu_{x y} \nu_{y x}}$ and $D_{66}=\frac{E_{x}\left(1-\nu_{x y}\right)}{2\left(1-\nu_{x y} \nu_{y x}\right)}$. $E_{x}$ and $E_{y}$ are the modulus of elasticity in the $\mathrm{x}$ and $\mathrm{y}$ directions respectively. $\nu$ is defined as Poisson's ratio.

The equations of harmonic motion in the frequency domain can be expressed in terms of the displacements $u(x, y)$ and $v(x, y)$ as

$$
\begin{aligned}
& D_{11}^{u} \frac{\partial^{2} u}{\partial x^{2}}+D_{66}^{u} \frac{\partial^{2} u}{\partial y^{2}}+\left(D_{12}^{u}+D_{66}^{u}\right) \frac{\partial^{2} v}{\partial x \partial y}+b_{x}=-\rho \omega^{2} u \\
& D_{22}^{u} \frac{\partial^{2}}{\partial x^{2}}+D_{66}^{u} \frac{\partial^{2} v}{\partial y^{2}}+\left(D_{12}^{u}+D_{66}^{u}\right) \frac{\partial^{2} u}{\partial x \partial y}+b_{y}=-\rho \omega^{2} v
\end{aligned}
$$

where $b_{x}$ and $b_{y}$ are body forces in $x$ and $y$ directions, respectively, $\rho$ the mass density per unit volume, and $\omega$ the circular frequency.

\subsubsection{Dispersion Relations}

In this section, the analytical frequency equation is presented based on a modified propagator matrix approach. 


\subsubsection{Solution}

The appropriate solution to the homogeneous equation, Equation 4.8 , in the absence of body forces, is [8]-[9]:

$$
\begin{aligned}
& u=j k\left(\Omega_{1}^{+}+b \Omega_{2}^{+}\right) e^{j k x} \\
& v=\left(a r_{1} \Omega_{1}^{-}+r_{2} \Omega_{2}^{-}\right) e^{j k x}
\end{aligned}
$$

where $j=\sqrt{-1}$, and

$$
\begin{aligned}
\Omega_{1}^{+} & =A_{11} \cos _{1} y+A_{12} \sin _{1} y, \\
\Omega_{1}^{-} & =A_{12} \operatorname{cosr}_{1} y-A_{11} \sin _{1} y, \\
\Omega_{2}^{+} & =A_{21} \cos r_{2} y+A_{22} \sin r_{2} y, \\
\Omega_{2}^{-} & =A_{22} \cos r_{2} y-A_{21} \sin _{2} y \\
a_{1} & =\left(k_{2}^{2}-\bar{\alpha} k^{2}-r_{1}^{2}\right) / \bar{\delta} r_{1}^{2}, \\
b_{1} & =\left(k_{2}^{2}-k^{2}-\bar{\beta} r_{2}^{2}\right) / \bar{\delta} k^{2} \\
\bar{\alpha}=\frac{D_{11}^{u}}{D_{66}^{u}} ; \bar{\beta} & =\frac{D_{22}^{u}}{D_{66}^{u}} ; k_{2}=\sqrt{\frac{\rho \omega^{2}}{D_{66}^{u}}} ; \bar{\delta}=1+\frac{D_{12}^{u}}{D_{66}^{u}}
\end{aligned}
$$

Here $r_{1}$ and $r_{2}$ are the roots of the equation

$$
\left|\begin{array}{cc}
r^{2}+\bar{\alpha} k^{2}-k_{2}^{2} & \bar{\delta} r^{2} \\
\bar{\delta} k^{2} & k^{2}-k_{2}^{2}+\bar{\beta} r^{2}
\end{array}\right|=0
$$

$A_{11}, A_{12}, A_{21}$ and $A_{22}$ are arbitrary constants for the plate and $k$ is the wavenumber. Stress and displacement components of the plate can be expressed in terms of these four unknown constants. By evaluating the stresses and displacements at $y=0$ and $y=L$, and performing algebraic manipulations, the following relation can be found:

$$
\mathrm{Q}_{L}=[P] \mathrm{Q}_{0}
$$

where

$$
\mathbf{Q}_{0}^{\mathbf{T}}=\left\langle\begin{array}{llll}
u_{0} & v_{0} & \sigma_{y y_{0}} & \sigma_{y x_{0}}
\end{array}\right\rangle .
$$


The vector $\mathbf{Q}_{0}$, which is unknown initially, represents the displacement and stress components at $y=0 .[P]$ is the propagator matrix for the plate whose elements are given in Appendix E.

By denoting the elements of the 4 by 4 matrix $[P]$ as $P_{m n}(m, n=1,2,3,4)$ and invoking the zero traction conditions at faces $y=0$ and $y=L$, the following relationship can be obtained from Equation 4.14:

$$
\left[\begin{array}{ll}
P_{31} & P_{32} \\
P_{41} & P_{42}
\end{array}\right]\left\{\begin{array}{l}
u_{1} \\
v_{1}
\end{array}\right\}=\left\{\begin{array}{l}
0 \\
0
\end{array}\right\} .
$$

Note that there is no external load acting on the plate. The dispersion equation (frequency equation) for the plate is obtained by setting the determinant of the coefficient matrix to zero i.e.

$$
f(\omega, k)=\left|\begin{array}{ll}
P_{31} & P_{32} \\
P_{41} & P_{42}
\end{array}\right|=0 .
$$

Let $\mathbf{Q}_{0 m}$ be $M$ number of modes to be evaluated and $k_{m}$ be the $m$ th root of Equation 4.17. Traction free conditions at face $y=L$ and Equation 4.16 give the components of the $m$ th eigenvector at face $y=0$, as

$$
\mathrm{Q}_{0 m}^{\mathrm{T}}=\left\langle 1, \quad-P_{31} / P_{32}, \quad 0, \quad 0\right\rangle .
$$

Then, applying Equation 4.14 at successive interfaces, the $m$ th modal eigenvector can be obtained as

$$
\mathrm{Q}_{m}^{\mathrm{T}}=\left\langle\mathrm{Q}_{0 m}^{\mathrm{T}}, \quad \mathrm{Q}_{L m}^{\mathrm{T}}\right\rangle
$$

where

$$
\mathrm{Q}_{0 m}^{\mathrm{T}}=\left\langle u_{0 m}, \quad v_{0 m}, \quad \sigma_{y y 0_{m}}, \quad \sigma_{y x_{0 m}}\right\rangle .
$$

The $u_{0 m}, v_{0 m}, \sigma_{y y_{0 m}}$ and $\sigma_{y x_{0 m}}$ are components of the $m$ th eigenvector determined at $y=0$. It may be noted that $\mathbf{Q}_{m}$ can be associated with propagating, non-propagating or evanescent modes corresponding to real, imaginary or complex $k_{m}$. 


\subsection{Analytical Method for Bending}

\subsubsection{General}

A simplified analytical solution to the classical bending problem is used to determine the accuracy of the Rayleigh-Ritz approximation for pure bending. The frequency equation is presented explicitly for a plate that is simply supported on all edges.

\subsubsection{Description of the problem}

In the analytical solution of the bending problem, for the purpose of checking only, it is assumed that all four edges of the plate are simply supported. This situation is shown in Figure 4.2. The plate has length, $\mathrm{a}$, in the $\mathrm{x}(=\mathrm{X})$ direction, width, $\mathrm{L}$, in the $\mathrm{y}(=\mathrm{Y})$ direction, and thickness, $\mathrm{h}$, in the $\mathrm{z}(=\mathrm{Z})$ direction. To simplify the expressions used in the derivation, the plate is assumed to be orthotropic and homogeneous. Also, the plate is positioned so that the global $X, Y, Z$ axes coincide with the local $x, y, z$ axes.

\subsubsection{Governing equations}

The strain-displacement relation is given by

$$
\{k\}=\left\{\begin{array}{c}
k_{x x} \\
k_{y y} \\
k_{x y}
\end{array}\right\}=\left\{\begin{array}{c}
-\frac{\partial^{2} w}{\partial x^{2}} \\
-\frac{\partial^{2} w}{\partial y^{2}} \\
-2 \frac{\partial^{2} w}{\partial x \partial y}
\end{array}\right\}
$$

where $w$ is the out-of-plane displacement (in the $z$ direction) and $k_{x x}, k_{y y}$ and $k_{x y}$ are the curvatures.

The moment-curvature relation is

$$
\{M\}=\left[D^{w}\right]\{\kappa\}
$$




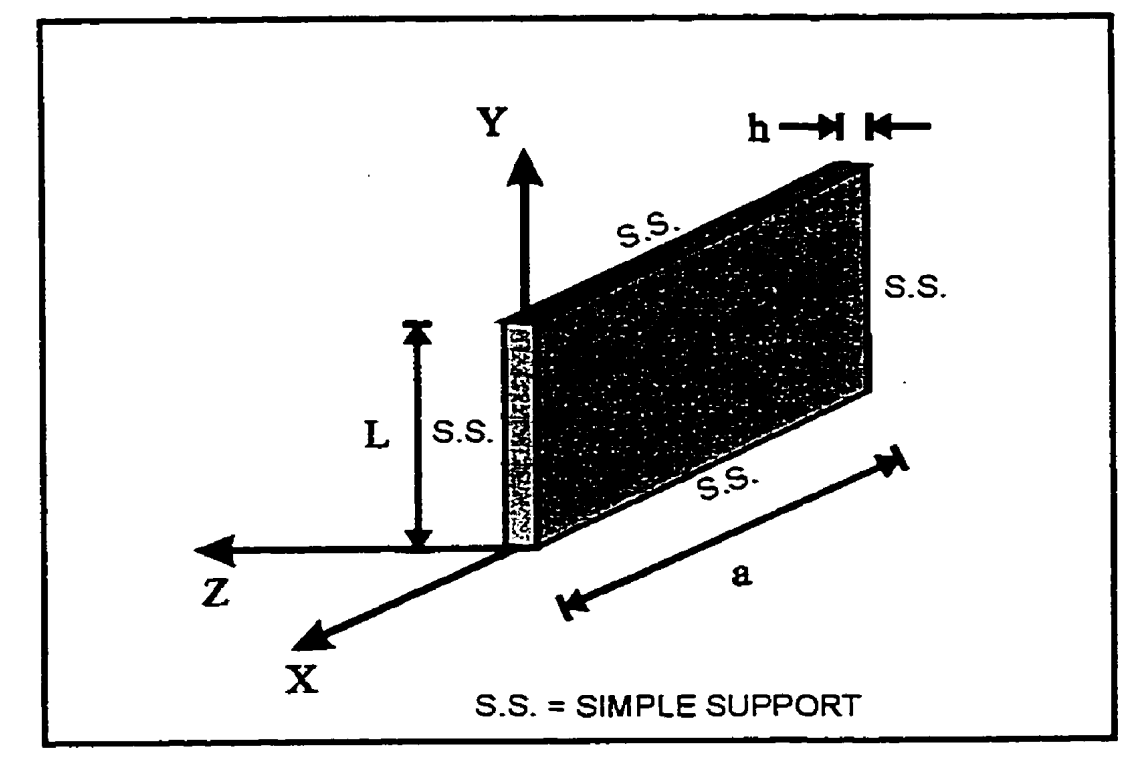

Figure 4.2: The geometry of a plate.

In Equation 4.22 the bending moments are $\{M\}=\left\{M_{x x}, M_{y y}, M_{x y}\right\}^{T}$. Here generalized plane stress conditions are assumed as

$$
\left[D^{w}\right]=\frac{h^{3}}{12}\left[\begin{array}{ccc}
D_{11} & D_{12} & 0 \\
D_{21} & D_{22} & 0 \\
0 & 0 & D_{66}
\end{array}\right]
$$

where $D_{11}=\frac{E_{x}}{1-\nu_{x y} \nu_{y x}}, D_{22}=\frac{E_{y}}{1-\nu_{x y} \nu_{y x}}, D_{12}=D_{21}=\frac{\nu_{x y} E_{y}}{1-\nu_{x y} \nu_{y x}}, D_{66}=\frac{E_{x}\left(1-\nu_{x y}\right)}{2\left(1-\nu_{x y} \nu_{y x}\right)}$.

The equations of harmonic motion in the frequency domain can be expressed in terms of $w(x, y, t)$ as

$$
D_{11}^{w} \frac{\partial^{4} w}{\partial x^{4}}+2\left(D_{12}^{w}+2 D_{66}^{w}\right) \frac{\partial^{4} w}{\partial x^{2} \partial y^{2}}+D_{66}^{w} \frac{\partial^{4} w}{\partial y^{4}}+b_{z}=-\rho h \frac{\partial^{2} w}{\partial t^{2}}
$$

where $b_{z}$ is the net body force in the $z$ direction and $\rho$ is the mass density [11]. 


\subsubsection{Solution}

For free vibrations, $b_{z}$, is set to zero. Given the boundary conditions shown in Figure 4.2, the appropriate solution of Equation 4.24 is:

$$
w(x, y, t)=\sum_{m} \sum_{n} A_{m n} \sin \left(\frac{m \pi x}{a}\right) \sin \left(\frac{n \pi y}{L}\right) e^{-i \omega t}
$$

where

$$
A_{m n}=\frac{\rho h}{D_{11}^{w}}\left[\left(\frac{m \pi}{a}\right)^{4}+2\left(\frac{D_{12}^{w}+2 D_{66}^{w}}{D_{11}^{w}}\right)\left(\frac{m \pi}{a}\right)^{2}\left(\frac{n \pi}{L}\right)^{2}+\frac{D_{66}^{w}}{D_{11}^{w}}\left(\frac{n \pi}{L}\right)^{4}\right]^{-1} .
$$

The frequency, $\omega$, is given by:

$$
\omega=\sqrt{\frac{D_{11}^{w}}{\rho h}}\left[\left(\frac{m \pi}{a}\right)^{4}+2\left(\frac{D_{12}^{w}+2 D_{66}^{w}}{D_{11}^{w}}\right)\left(\frac{m \pi}{a}\right)^{2}\left(\frac{n \pi}{L}\right)^{2}+\frac{D_{66}^{w}}{D_{11}^{w}}\left(\frac{n \pi}{L}\right)^{4}\right]^{\frac{1}{2}}
$$

\subsection{Summary}

The analytical solutions presented in this chapter will be used later in the study to assess the accuracy of the two-dimension and three-dimension Rayliegh-Ritz approximations presented earlier. 


\section{Chapter 5}

\section{Numerical Results}

\subsection{Introduction}

In this chapter the numerical accuracy of the Rayleigh-Ritz aproximations will be discussed for wave propagation and free vibration problems.

\subsection{Wave Propagation Problem}

Two approximate stiffness methods were formulated in the previous chapters using the two-dimensional analysis for thin-walled structural members and a three-dimensional analysis. Two analytical solutions were also presented for the inplane motion and bending motion of a thin plate. In order to check the two-dimensional stiffness approximation's ability to model the behaviour of a cross-section comprised of thin plates, it must be proved that the stiffness approximation can be used to model the behaviour of a single thin plate. The two-dimension Rayleigh-Ritz approximation is based on the knowledge that, for a thin plate, the inplane motions and bending 
motions are uncoupled. Thus, the first check of the two-dimensional stiffness approximation was made by comparing the results from the analytical solutions for the bending and inplane motions of a thin plate.

A thin plate was analyzed using the two-dimensional Rayleigh-Ritz approximation and the inplane analytical solution. The bending problem will be evaluated separately. The height of the plate is $10 \mathrm{~cm}$, the thickness is $1 \mathrm{~cm}$. It was assumed for simplicity that the plate was homogenous, isotropic and infinitely long in the longitudinal direction with $E=2 \frac{2}{3} \frac{\mathrm{kg} \mathrm{m}}{\mathrm{s}^{2}}$ and $\nu=\frac{1}{3}$. For a wavenumber, $\gamma=0.00000+0.3141593 i$, the analysis obtains the circular frequencies, $\omega \mathrm{rad} / \mathrm{s}$ shown in Table 5.1. 30 elements were used in the 2D Rayleigh-Ritz approximation.

\begin{tabular}{|c|c|c|}
\cline { 2 - 3 } \multicolumn{1}{c|}{} & Two-Dimensional RR & Analytical Inplane \\
\hline 1 & 0.23928 & 0.23928 \\
\hline 2 & 0.44425 & 0.44425 \\
\hline 3 & 0.58395 & 0.58395 \\
\hline 4 & 0.59352 & 0.59352 \\
\hline 5 & 0.85868 & 0.85868 \\
\hline
\end{tabular}

Table 5.1: A comparison of the two-dimensional stiffness approximation and inplane analytical method for calculating circular frequencies of wave propagation, $\mathrm{rad} / \mathrm{s}$, in a plate.

The two-dimensional approximation and the analytical inplane method give identical resuits for the wave propagation problem. For clarity the frequencies corresponding to modes of pure bending have been omitted.

Next, a thin plate was analyzed using the two-dimensional stiffness approximation and the analytical bending solution. To model the bending problem the two-dimensional stiffness approximation was changed, by eliminating the rows and columns in the structure equations associated with the displacment of the plate in 
the $z$ direction at nodes $y=0$ and $y=L$. With these rows and columns eliminated the two-dimensional stiffness approximation models a plate simply supported at its edges $y=0$ and $y=L$. The height of the plate is $6 \mathrm{~cm}$, the thickness is $1 \mathrm{~cm}$. It was assumed, for simplicity, that the plate was homogenous, isotropic and infinitely long in the longitudinal direction with a density $\gamma_{1} g=1 \frac{\mathrm{kg}}{\mathrm{m}^{3}}$, Young's Modulus is $1 \frac{\mathrm{kg} \mathrm{m}}{\mathrm{s}^{2}}$ and Poisson's ratio, $\nu=0$. For a wavenumber, $\gamma=0.00000+0.10472 i$, the analysis obtains the circular frequencies, $\omega \mathrm{rad} / \mathrm{s}$ shown in Table 5.2. 30 elements were used in the 2D Rayleigh-Ritz approximation.

\begin{tabular}{|c|c|c|}
\cline { 2 - 3 } \multicolumn{1}{c|}{} & Two-Dimensional RR & Analytical Bending \\
\hline 1 & 0.0823076 & 0.082308 \\
\hline 2 & 0.319733 & 0.319733 \\
\hline 3 & 0.715443 & 0.715443 \\
\hline 4 & 1.26944 & 1.269437 \\
\hline 5 & 1.98172 & 1.981714 \\
\hline
\end{tabular}

Table 5.2: A comparison of the two-dimensional stiffness approximation and bending analytical method for calculating circular frequencies of wave propagation, $\mathrm{rad} / \mathrm{s}$, in a plate.

The two-dimensional approximation and the analytical solution to the bending problem give near identical results for the wave propagation problem. For clarity the frequencies corresponding to the modes of inplane motion have been omitted.

Next, the two-dimensional stiffness approximation was compared to the threedimensional stiffness approximation for the wave propagation problem. A thin plate was analyzed using both two-dimensional and three-dimensional stiffness approximations. The height of the plate is $10 \mathrm{~cm}$, the thickness is $1 \mathrm{~cm}$. It is assumed for simplicity that the plate is homogenous, isotropic and infinitely long in the longitudinal direction with $E=2 \frac{2}{3} \frac{\mathrm{kg} \mathrm{m}}{\mathrm{s}^{2}}$ and $\nu=\frac{1}{3}$. For a wavenumber, $\gamma=0.00000+0.3141593 i$, 
the analysis obtains the circular frequencies, $\omega \mathrm{rad} / \mathrm{s}$ shown in Table 5.3. 30 elements were used in the 2D Rayleigh-Ritz approximation and 30 4-node elements were used in the 3D Rayleigh-Ritz approximation.

\begin{tabular}{|c|c|c|c|c|}
\cline { 2 - 5 } \multicolumn{1}{c|}{} & \multicolumn{2}{c|}{ Two Dimensional RR } & \multicolumn{2}{c|}{ Three Dimensional RR } \\
\cline { 2 - 5 } \multicolumn{1}{c|}{} & $\theta=0^{\circ}$ & $\theta=30^{\circ}$ & $\theta=0^{\circ}$ & $\theta=30^{\circ}$ \\
\hline 1 & 0.04784 & 0.04784 & 0.05111 & 0.05111 \\
\hline 2 & 0.07940 & 0.07940 & 0.07875 & 0.07875 \\
\hline 3 & 0.18209 & 0.18209 & 0.18547 & 0.18547 \\
\hline 4 & 0.23928 & 0.23928 & 0.23943 & 0.23943 \\
\hline 5 & 0.37509 & 0.37509 & 0.37240 & 0.37240 \\
\hline 6 & 0.44425 & 0.44425 & 0.44430 & 0.44430 \\
\hline 7 & 0.58395 & 0.58395 & 0.58346 & 0.58346 \\
\hline 8 & 0.59352 & 0.59352 & 0.59351 & 0.59351 \\
\hline 9 & 0.66746 & 0.66746 & 0.62138 & 0.62138 \\
\hline 10 & 0.85868 & 0.85868 & 0.85824 & 0.85824 \\
\hline
\end{tabular}

Table 5.3: A comparison of the 2D stiffness approximation and 3D stiffness approximation for calculating circular frequencies of wave propagation, $\mathrm{rad} / \mathrm{s}$, in a plate.

In both cases the results of the stiffness approximations were nearly identical to the analytical solution. A comparison of the frequencies and corresponding mode shapes (not shown) calculated using the two-dimensional Rayleigh-Ritz approximation and the three-dimensional Rayleigh-Ritz approximation shows that, for a straight thin plate, the two-approximate methods are almost identical. As expected, the mode shapes from both models indicate that the frequencies can be divided into modes of pure inplane motion and bending motion. Notice that when the same plate is analyzed again when the plate is rotated at an angle, $\theta$, from the global $Y$ axis the frequencies of both approximate analyses do not change. The only change that occurs is that the directions of the mode shapes are rotated an angle, $\theta$, from the global $Y$ axis. This revelation is important because it proves that the motion of the plate is 
independent of the angle $\theta$, which is crucial when the plates are arranged to form various structural shapes.

The two-approximate stiffness methods that were formulated earlier were checked next against the analytical solution for the propagation of waves through a cylinder of infinite length. The formulation for the analytical solution of a cylinder will not be presented, only the numerical results will be presented. However, the theory has been presented by Zhuang [12].

The cylinder was analyzed using both the two-dimensional and the three-dimensional stiffness approximations as well as the analytical solution. The radius of the cylinder analyzed was assumed to be $10 \mathrm{~cm}$ and the thickness was $1 \mathrm{~cm}$. It was assumed, for simplicity, that the cylinder was homogenous and isotropic, with $E=2 \frac{2}{3} \frac{\mathrm{kg} \mathrm{m}}{\mathrm{s}^{2}}$ and $\nu=\frac{1}{3}$. For a wave number, $\gamma=0.00000+0.20000 i$, the analysis obtains the circular frequencies, $\omega \mathrm{rad} / \mathrm{s}$, shown in Table 5.4. 300 elements were used in the $2 \mathrm{D}$ Rayleigh-Ritz approximation and 60 9-node elements were used in the 3D RayleighRitz approximation.

A comparison of the lowest eleven frequencies and corresponding mode shapes (not shown) shows that the two approximate methods give nearly identical solutions. The slight variance between the two approximate solutions is most likely due to the use of three-dimensional curved elements to model the curved surface. In the twodimensional solution a curved surface is modeled using straight elements. Thus, it comes as no suprise that the analytical solution is closer to the three-dimensional stiffness approximation than the two-dimensional stiffness approximation.

Please note that for each frequency that was calculated using the stiffness approximations there should be a corresponding frequency calculated when using the analytical solution. The remainder of the analytical frequencies could have been obtained through repeated solution of the analytical problem by increasing the number 


\begin{tabular}{|c|c|c|c|}
\cline { 2 - 4 } \multicolumn{1}{c|}{} & Two-Dimensional & Three-Dimensional & Analytical Solution \\
\hline 1 & 0.07325 & 0.07271 & 0.07250 \\
\hline 2 & 0.08102 & 0.08096 & 0.08093 \\
\hline 3 & 0.09617 & 0.09437 & \\
\hline 4 & 0.11949 & 0.11941 & 0.11941 \\
\hline 5 & 0.13780 & 0.13370 & \\
\hline 6 & 0.16168 & 0.16131 & 0.16131 \\
\hline 7 & 0.19183 & 0.18398 & \\
\hline 8 & 0.20028 & 0.20000 & 0.20000 \\
\hline 9 & 0.24192 & 0.24135 & 0.24135 \\
\hline 10 & 0.25649 & 0.24305 & \\
\hline 11 & 0.30050 & 0.29988 & 0.29986 \\
\hline
\end{tabular}

Table 5.4: A comparison of the 2D stress stiffness approximation, 3D stiffness approximation and analytical solution by showing computed circular frequencies of wave propagation, $\omega \mathrm{rad} / \mathrm{s}$, in a cylinder.

of circumferential waves used in each solution. However, for this report it was decided that further calculations were not necessary and only the results for the number of circumferential waves, $m=0,1,2,3$, have been presented.

\subsection{The Vibration of Structural Members Simply Supported at Their Ends}

The two stiffness methods can be used to calculate all of the modes of vibration of a simply supported structural member. In this thesis simplified solutions are used to check the numerical accuracy of the stiffness methods. However, only some of the modes of vibration have simplified solutions. In this context, simplified solutions are defined as the solution to the classical beam equation and the solution to 
one-dimensional longitudinal vibration problems. The modes of vibration that have simplified solutions are free transverse vibration and free longitudinal vibration.

\section{Free Transverse Vibration}

The characteristic frequencies of free transverse vibration for a structural member, simply supported at its end, can be calculated using simple solutions.

The characteristic frequencies of a simply supported member are [11]

$$
\hat{\omega}_{n}=\frac{n^{2} \pi^{2}}{a^{2}} \sqrt{\frac{E I g}{\gamma_{1} \Omega}} \quad(\mathrm{n}=1,2, \ldots)
$$

where $a$ is the distance between supports, $I$ is the moment of inertia of the member, $g$ is the acceleration of gravity, $\Omega$ is the cross-sectional area, $\gamma_{1}$ is the mass per unit volume of material and $n$ is the number of half wavelengths in the mode of vibration being solved.

\section{Free Longitudinal Vibration}

If the two stiffness methods are used to calculate all the modes of free vibration, there should be a mode of free longitudinal vibration which occurs when both ends of the beam are free to move in the longitudinal direction. The characteristic frequencies of vibration of these modes can be calculated using simplified solutions. The simplified solution can be expressed as [11]

$$
\hat{\omega}_{n}=\frac{n \pi}{a} \sqrt{\frac{E g}{\gamma_{1}}} \quad(\mathrm{n}=0,1,2, \ldots)
$$

where, $a$ is the distance between supports, $g$ is the acceleration of gravity and $\gamma_{1}$ is the mass per unit volume of material.

In order to verify that the two-dimensional Rayleigh-Ritz approximation can model the free vibration of a thin-walled structural member which is simply supported at its ends, it must be shown that the approximation can model the free 
vibration of a thin plate simply supported at its ends. Consider a plate whose height is $6 \mathrm{~cm}$, thickness is $1 \mathrm{~cm}$ and its unsupported length is $30 \mathrm{~cm}$. It is assumed, for convenience, that the plate has a density $1 \frac{\mathrm{kg}}{\mathrm{m}^{3}}$ and Young's Modulus is $1 \frac{\mathrm{kg} m}{\mathrm{~s}^{2}}$. The first fifteen frequencies of vibration of the beam, calculated using the two-dimensional Rayleigh-Ritz approximation are shown in Table 5.5 in ascending order for the number of half wavelengths, $n=1,2,3,4,5$. The simplified solutions for free longitudinal vibration, free out of plane transverse vibration $(x-z)$ and free inplane transverse vibration $(x-y)$ are used to calculate the circular frequencies shown in Tables 5.6, 5.7 and 5.8. 30 elements were used in the 2D Rayleigh-Ritz approximation.

\begin{tabular}{|c|c|c|c|c|c|}
\cline { 2 - 6 } \multicolumn{1}{c|}{} & \multicolumn{5}{c|}{ 2D Rayleigh-Ritz Approximation } \\
\cline { 2 - 6 } \multicolumn{1}{c|}{} & $\mathrm{n}=1$ & $\mathrm{n}=2$ & $\mathrm{n}=3$ & $\mathrm{n}=4$ & $\mathrm{n}-5$ \\
\hline 1 & 0.00316568 & 0.0126627 & 0.0284911 & 0.0506508 & 0.0791419 \\
\hline 2 & 0.0178275 & 0.0506311 & 0.0783530 & 0.108978 & 0.143392 \\
\hline 3 & 0.0248398 & 0.0637460 & 0.124134 & 0.191165 & 0.261092 \\
\hline 4 & 0.104702 & 0.205733 & 0.235096 & 0.271913 & 0.314560 \\
\hline 5 & 0.186291 & 0.209431 & 0.314153 & 0.418875 & 0.523592 \\
\hline 6 & 0.389888 & 0.440453 & 0.508587 & 0.511651 & 0.523595 \\
\hline 7 & 0.500106 & 0.513066 & 0.508797 & 0.578584 & 0.622075 \\
\hline 8 & 0.520085 & 0.516525 & 0.543033 & 0.586022 & 0.668460 \\
\hline 9 & 0.752563 & 0.785290 & 0.832226 & 0.888436 & 0.950795 \\
\hline 10 & 0.974380 & 0.988941 & 1.01075 & 1.00534 & 1.00731 \\
\hline 11 & 1.03785 & 1.02247 & 1.01291 & 1.04586 & 1.08728 \\
\hline 12 & 1.12606 & 1.15939 & 1.20087 & 1.24768 & 1.29854 \\
\hline 13 & 1.47724 & 1.46943 & 1.46242 & 1.45882 & 1.45992 \\
\hline 14 & 1.57909 & 1.60049 & 1.63007 & 1.66514 & 1.70431 \\
\hline 15 & 1.60713 & 1.62061 & 1.64293 & 1.67390 & 1.71326 \\
\hline
\end{tabular}

Table 5.5: The circular frequencies of vibration, $\mathrm{rad} / \mathrm{s}$, for a simply supported plate calculated using two-dimensional stiffness method.

Comparing the values calculated using the two-dimensional Rayleigh-Ritz approx- 


\begin{tabular}{|c|c|c|c|c|}
\hline \multicolumn{5}{|c|}{ Free Longitudinal Vibration - Analytical } \\
\hline $\mathrm{n}=1$ & $\mathrm{n}=2$ & $\mathrm{n}=3$ & $\mathrm{n}=4$ & $\mathrm{n}=5$ \\
\hline 0.1047198 & 0.2094395 & 0.3141593 & 0.4188790 & 0.5235988 \\
\hline
\end{tabular}

Table 5.6: The free longitudinal circular frequencies of vibration, $\mathrm{rad} / \mathrm{s}$, of a simply supported plate calulated using the simplified solution.

\begin{tabular}{|c|c|c|c|c|}
\hline \multicolumn{5}{|c|}{ Transverse Vibration - Out of Plane (x-z) - Analytical } \\
\hline $\mathrm{n}=1$ & $\mathrm{n}=2$ & $\mathrm{n}=3$ & $\mathrm{n}=4$ & $\mathrm{n}=5$ \\
\hline 0.00316568 & 0.0126627 & 0.0284911 & 0.0506508 & 0.0791419 \\
\hline
\end{tabular}

Table 5.7: The free, out of plane, transverse circular frequencies of vibration, $\mathrm{rad} / \mathrm{s}$, of a simply supported plate calculated using the simplified solution.

\begin{tabular}{|c|c|c|c|c|}
\hline \multicolumn{4}{|c|}{ Transverse Vibration - In Plane (x-y)- Analytical } \\
\hline $\mathrm{n}=1$ & $\mathrm{n}=2$ & $\mathrm{n}=3$ & $\mathrm{n}=4$ & $\mathrm{n}=5$ \\
\hline 0.0189941 & 0.0759763 & 0.1709466 & 0.3039051 & 0.4748517 \\
\hline
\end{tabular}

Table 5.8: The free inplane transverse circular frequencies of vibration, $\mathrm{rad} / \mathrm{s}$, of a simply supported plate calculated using the simplified solution. 


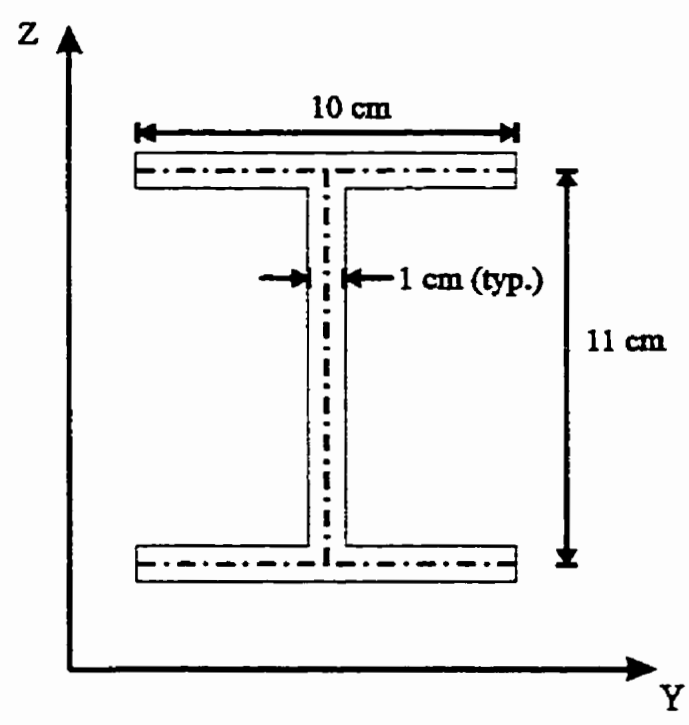

Figure 5.1: The geometry of the I shaped section in the global coordinate system.

imation and the corresponding simplified solutions reveals good agreement for both the free longitudinal and free out of plane, transverse modes of vibration. But, a comparison of the two-dimensional stiffness solution and the simplified solution for the free inplane transverse modes of vibration shows some disagreement. However, it is expected that the approximate solutions will not agree with the simplified solution. The approximate stiffness solutions both take into account the distortion of the crosssection geometry during vibration, whereas the simplified solution assumes that the geometry of the cross-section does not change. As a result, the approximate stiffness solution is more indicative of the true behaviour of the simply supported plate.

Next, the I-shaped member simply supported at its ends, which is shown in Figure 5.1, was analyzed using the two-dimensional stiffness approximation, the threedimensional stiffness approximation and the corresponding simplified solutions for bending modes. It was assumed for simplicity that the section was homogenous and isotropic, with $E=2 \frac{2}{3} \frac{\mathrm{kgm}}{\mathrm{s}^{2}}$ and $\nu=\frac{1}{3}$. The frequencies of the first characteristic 
mode of vibration in the $\mathrm{X}-\mathrm{Z}$ plane and the $\mathrm{X}-\mathrm{Z}$ plane of the member are shown in Tables 5.9 and 5.10. 30 elements were used in the 2D Rayleigh-Ritz approximation and 120 4-node elements were used in the 3D Rayleigh-Ritz approximation.

\begin{tabular}{|l|c|c|c|}
\cline { 2 - 4 } \multicolumn{1}{c|}{} & Two-Dimensional & Three-Dimensional & Simplified Solution \\
\hline$\hat{\omega}_{1 y y} \mathrm{rad} / \mathrm{s}$ & 0.10425 & 0.11338 & 0.08316 \\
\hline
\end{tabular}

Table 5.9: A comparison of the 2D stiffness approximation, 3D stiffness approximation and analytical solution by showing computed circular frequencies of free vibration in the $\mathrm{X}-\mathrm{Z}$ plane of an I shaped member, simply supported at its ends.

\begin{tabular}{|c|c|c|c|}
\cline { 2 - 4 } \multicolumn{1}{c|}{} & Two-Dimensional & Three-Dimensional & Simplified Solution \\
\hline$\hat{\omega}_{1 z z} \mathrm{rad} / \mathrm{s}$ & 0.03772 & 0.03872 & 0.04971 \\
\hline
\end{tabular}

Table 5.10: A comparison of the 2D stiffness approximation, 3D stiffness approximation and analytical solution by showing computed circular frequencies of free vibration in the X-Y plane of an I shaped member, simply supported a.t its ends.

Again, the simplified solutions do not give good agreement with the two-dimensional stiffness approximation or the three-dimensional stiffness approximation. However, it is reiterated that the two-dimensional stiffness approximation and the three-dimensional stiffness approximation are a better model of the actual behaviour of the member. Both stiffness approximations allow for the distortion of the cross-section geometry during vibration whereas the simplified solution models the vibration of the member with the geometry of the section remaining unchanged. The distortion of the cross-section geometry during vibration is visble in the modeshapes, shown in Figures 5.2 and 5.3 which correspond to the aforementioned frequencies. 
(a)

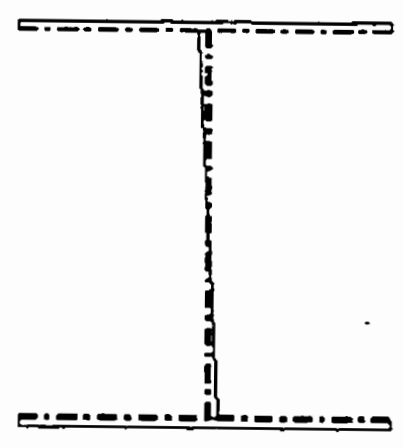

$\mathbf{U}$ (b)

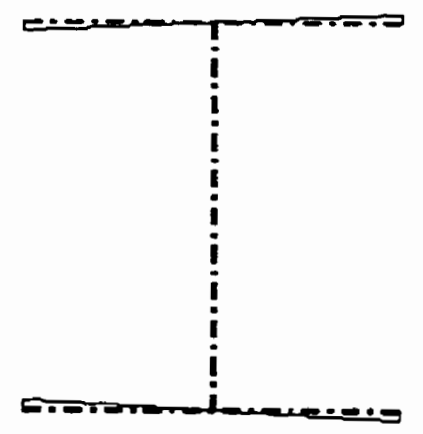

V (c)

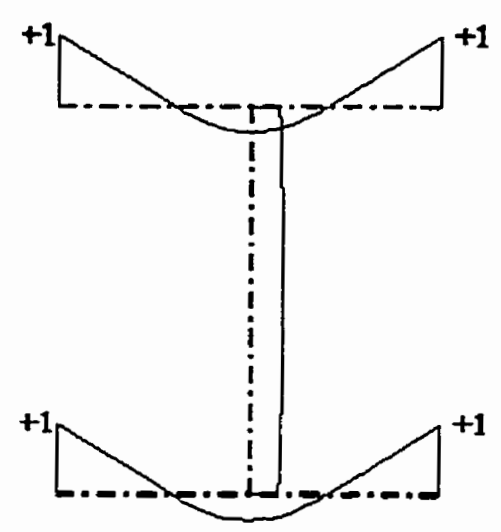

$\mathbf{w}$

Figure 5.2: A sketch of the mode shape corresponding to $\omega_{1 y y}$.

(a)

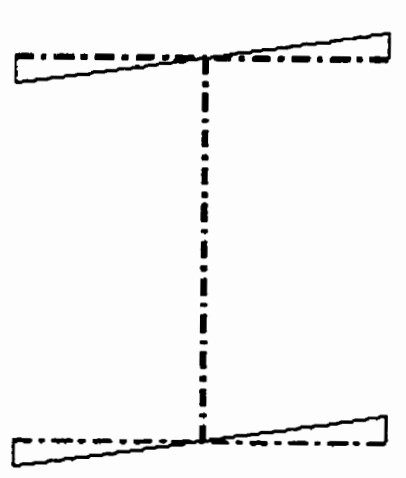

U (b)

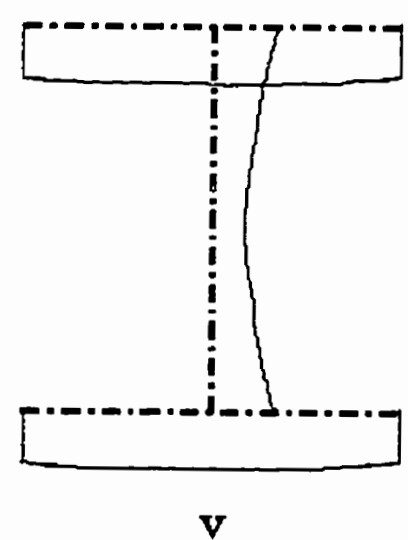

(c)

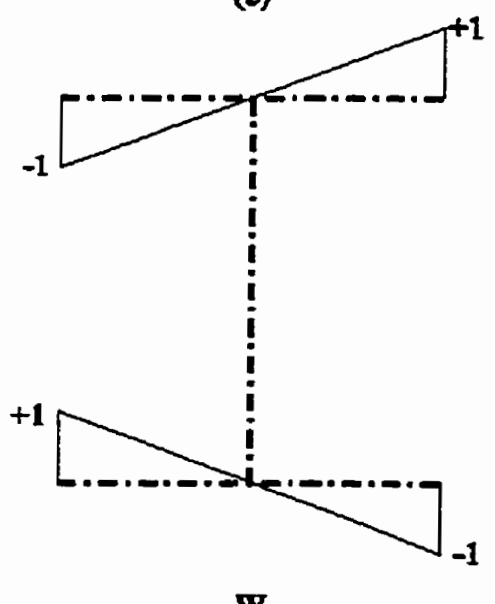

$\mathbf{w}$

Figure 5.3: A sketch of the mode shape corresponding to $\omega_{1 z z}$. 


\subsection{Free Transverse Vibration of Rectangular Plates Simply Supported on Four Edges}

In this section the two-dimensional stiffness approximation for the vibration of a plate, simply supported at its ends, is modified to model the vibration of a plate simply supported on all four edges. The stiffness approximation of a plate simply supported at its ends is made to model the vibration of a plate simply supported on all four of its edges by setting the displacement of the two edge nodes, $y=0$ and $y=L$ to zero in the Y-direction.

The characteristic frequencies of vibration of a structural member simply supported along its edges can be derived using simple solutions. The characteristic frequencies for the transverse vibration of a plate simply supported along its edges are given by

$$
\hat{\omega}_{m n}=\pi^{2} \sqrt{\frac{E I g}{\left(1-\nu^{2}\right) \gamma_{1} h}}\left(\frac{m^{2}}{L^{2}}+\frac{n^{2}}{a^{2}}\right)
$$

where $L$ is the width, $a$ is the length, $h$ is the thickness, $I=\frac{h^{3}}{12}$, is the moment of inertia of a unit width of cross section, $g$ is the acceleration of gravity, $\nu$ is Poisson's ratio, $E$ is Young's Modulus and $\gamma_{1}$ is the specific weight of the plate.

Consider a plate whose width is $6 \mathrm{~cm}$, thickness is $1 \mathrm{~cm}$ and its unsupported length is $30 \mathrm{~cm}$. It is assumed, for convenience, that the plate has a density $\gamma_{1} g=1 \frac{\mathrm{kg}}{\mathrm{m}^{3}}$, Young's Modulus is $1 \frac{\mathrm{kgm}}{\mathrm{s}^{2}}$ and Poisson's ratio, $\nu=0$. The simplified solution, Equation 5.3, for the transverse vibration of a plate simply supported along its edges is used to calculate the characteristic frequencies shown in Table 5.11. The same problem using the two-dimensional approximation finds the frequencies of all possible modes of vibration including those of the free transverse plate vibration, free longitudinal vibration and inplane, transverse vibration of a simple plate. For simplicity, only the modes corresponding to free transverse plate vibration are shown in Table 5.12, 
the remaining modes have already been presented. 30 elements were used in the $2 \mathrm{D}$ Rayleigh-Ritz approximation.

\begin{tabular}{|c|c|c|c|c|c|}
\cline { 2 - 6 } \multicolumn{1}{c|}{} & \multicolumn{5}{c|}{ Free Transverse Vibration of a } \\
\cline { 2 - 6 } \multicolumn{1}{c|}{} & $\mathrm{n}=1$ & $\mathrm{n}=2$ & $\mathrm{n}=3$ & $\mathrm{n}=4$ & $\mathrm{n}=5$ \\
\hline $\mathrm{m}=1$ & 0.082308 & 0.091805 & 0.107633 & 0.129793 & 0.158284 \\
\hline $\mathrm{m}=2$ & 0.319733 & 0.329230 & 0.345059 & 0.367219 & 0.395710 \\
\hline $\mathrm{m}=3$ & 0.715443 & 0.724940 & 0.740768 & 0.762928 & 0.791419 \\
\hline $\mathrm{m}=4$ & 1.269437 & 1.278934 & 1.294762 & 1.316922 & 1.345413 \\
\hline $\mathrm{m}=5$ & 1.981714 & 1.991211 & 2.007039 & 2.029199 & 2.057690 \\
\hline
\end{tabular}

Table 5.11: The free, out of plane, transverse circular frequencies of vibration, $\mathrm{rad} / \mathrm{s}$, of a rectangular plate, simply supported along all four edges, calculated using the simplified solution.

\begin{tabular}{|c|c|c|c|c|c|}
\cline { 2 - 6 } \multicolumn{1}{c|}{} & \multicolumn{4}{c|}{ Two-Dimensional Stiffness Approximation } \\
\cline { 2 - 6 } \multicolumn{1}{c|}{} & $\mathrm{n}=1$ & $\mathrm{n}=2$ & $\mathrm{n}=3$ & $\mathrm{n}=4$ & $\mathrm{n}=5$ \\
\hline $\mathrm{m}=1$ & 0.0823076 & 0.0918046 & 0.107633 & 0.129793 & 0.158284 \\
\hline $\mathrm{m}=2$ & 0.319733 & 0.329230 & 0.345059 & 0.367219 & 0.395710 \\
\hline $\mathrm{m}=3$ & 0.715443 & 0.724940 & 0.740769 & 0.762928 & 0.791420 \\
\hline $\mathrm{m}=4$ & 1.26944 & 1.27894 & 1.294762 & 1.31692 & 1.34541 \\
\hline $\mathrm{m}=5$ & 1.98172 & 1.99122 & 2.00705 & 2.02921 & 2.05770 \\
\hline
\end{tabular}

Table 5.12: The circular frequencies of vibration, $\mathrm{rad} / \mathrm{s}$, of a plate, simply supported along all four edges, calculated using the two-dimensional stiffness method.

From the values tabulated in Tables 5.11 and 5.12 it is easy to see that the twodimensional stiffness approximation and the simplified solution agree quite closely with one another. The calculated frequencies of vibration are almost identical. 


\subsection{Numerical Difficulties at High Frequencies}

As with any Rayleigh-Ritz stiffness approximation that requires the solution of an eigenvalue problem, the accuracy of the solution decreases as frequency increases, however by increasing the number of elements used in the solution the accuracy can be increased. But, increasing the number of elements also increases the size of the eigenvalue problem being solved. Thus, the computational time required to solve the larger problem increases significantly and so does the demand on the memory of the computer solving the problem. That being said, the stiffness approximation is still an efficient way to calculate wave dispersion characteristics.

\subsection{Summary}

In this chapter the two-dimensional stiffness approximation was proven to be effective in calculating the wave dispersion characteristics of thin-walled, infinitely long members as well as the characteristic frequencies of vibration of simply supported thin-walled structural members and simply supported thin plates. However, there are numerical difficulties when calculating the wave dispersion characteristics of very high frequencies using the Rayleigh-Ritz type stiffness approximations. 


\section{Chapter 6}

\section{Conclusions}

\subsection{Concluding Remarks}

The purpose of this study was to create a multi-purpose program that can analyzed thin-walled structural members using a two dimensional Rayleigh-Ritz stiffness approximation. The program that was created met all expectations, the numerical results prove that the program can be used to calculate the wave dispersion characteristics of the wave propagation problem and characteristic frequencies of vibration of simply supported members and plates simply supported along four edges with great accuracy. However, it should be noted that like any Rayleigh-Ritz approximation of this type numerical difficulties arise when solving the eigenvalue problem for high frequencies of vibration.

\subsection{Future Work}

The next step in developing a quantitative non-destructive evaluation method is to create a program to do a scattering analysis on thin-walled structural members using 
the wave dispersion characteristics calculated using the methodology described in this report.

The versatility of the finite element method makes many different types of analysis possible. The list of possible future work on the vibration of beams could include changes in the support conditions and applying external forces to nodes to create forced vibration etc.. The static analysis of beams compensating for the deformation of the cross-section geometry under deflection is also a possibility, ie. when the body forces are not equal to zero but the frequency of "vibration" is zero. 


\section{REFERENCES}

[1] W.M. Karusena, A.H. Shah, and S.K. Datta, "Elastic Wave Propagation in Laminated Composite Plates" Journal of Engineering Materials and Technology, ASME, Vol. 113, 1991, pp 411-418.

[2] W.M. Karusena, A.H. Shah, and S.K. Datta, "Wave Propagation in a MultiLayered Laminated Cross-Ply Composite Plate." Journal of Applied Mechanics, ASME, Vol. 58, 1992, pp 1028-1032.

[3] S.K. Datta, A.H. Shah, and W.M. Karusena, "Edge and Layering Effects in a Multi-Layered Composite Plate." Computers and Structures, Vol. 37, No. 2, 1990, pp 151-162.

[4] W.M. Karusena, R.L. Bratton, A.H. Shah, and S.K. Datta, "Reflection of Plane Strain Waves at the Free Edge of a Laminated Composite Plate." International Journal of Solids and Structures, Vol. 27, No.8, 1991, pp 949-964.

[5] E. Pan, J. Rogers, S.K. Datta and A.H. Shah, "Mode selection of guided waves for ultrasonic inspection of gas pipelines with thick coating." Mechanics of Materials, Vol. 31, 1999, pp 165-174.

[6] J. Zhu and A.H. Shah, "Modal Representation of Transient Dynamics of Laminated Plate.", Journal of Engineering Mechanics, ASCE, Vol.121, 1995, pp 26-36 
[7] S.B. Dong and M. Kazic, "End Modes and Their Application to Wave Reflection Analysis", Dept. of Civil Eng., University of California, Los Angeles, California.

[8] W.M. Karusena, "Elastic Waves in Laminated Composite Plates for Application in Ultrasonic Nondestructive Evaluation", Ph.D. Thesis, Dept. of Civil Eng., University of Manitoba, Winnipeg, Manitoba, Canada, 1992.

[9] J. Zhu, "Numerical Modeling for Elastic Waves in Laminated Composite Plates", Ph.D. Thesis, Dept. of Civil Eng., University of Manitoba, Winnipeg, Manitoba, Canada, 1996.

[10] Klaus-Jurgen Bathe, "Finite Element Procedures", Prentice Hall Publishers, 1996, pp.344.

[11] Graff, Karl F. (1975), "Wave motion in elastic solids.", Ohio State University Press, pp. 233.

[12] W. Zhuang, "Numerical Modeling for Elastodynamic Problems in Laminated Composite Cylinders", Ph.D. Thesis, Dept. of Civil Eng., University of Manitoba, Winnipeg, Manitoba, Canada, 1999. 


\section{Appendix A}

\section{Matrices of the 2D Wave}

\section{Propagation Problem}

The elements of $[M],\left[K_{1}\right],\left[K_{2}\right],\left[K_{3}\right],\left[M_{b}\right],\left[E_{1}\right],\left[E_{3}\right]$ and $\left[E_{5}\right]$ appearing in Equation 2.48 are:

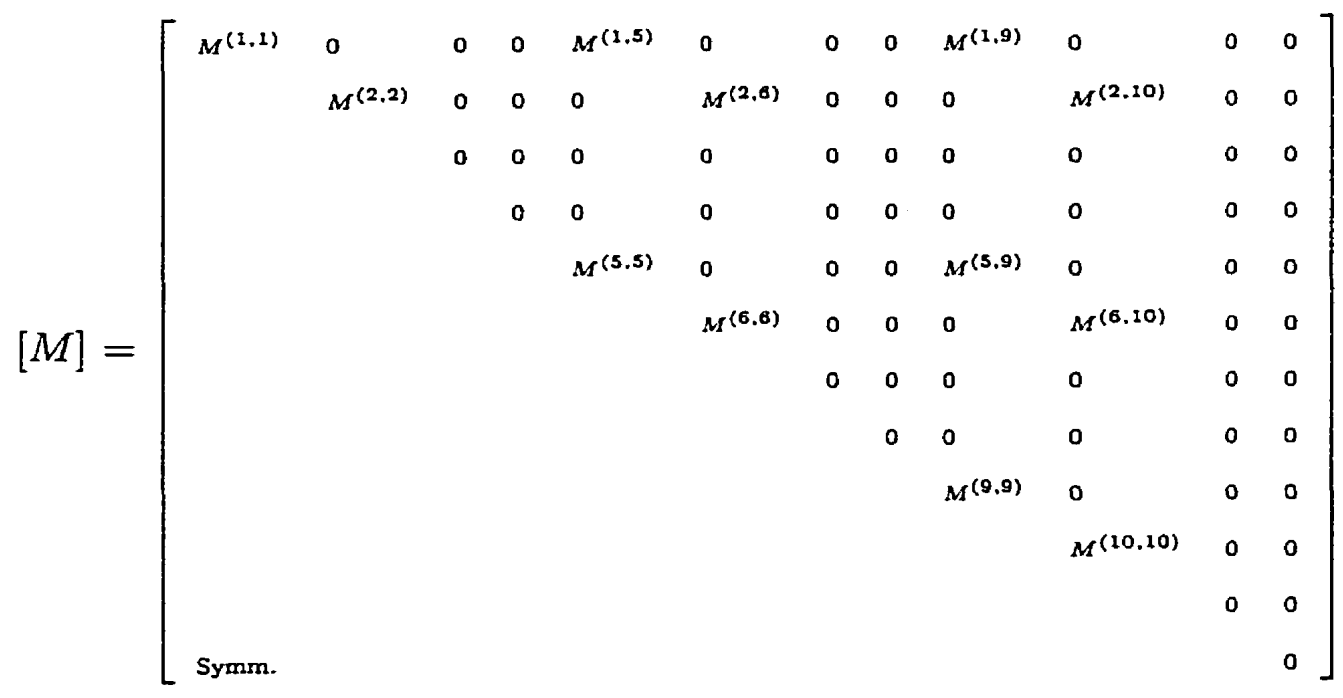

where

$$
\begin{array}{lll}
M^{(1,1)}=\frac{2}{15} \rho L_{u}, & M^{(2,2)}=\frac{2}{15} \rho L_{u}, & M^{(1,5)}=\frac{1}{15} \rho L_{u}, \\
M^{(2,6)}=\frac{1}{15} \rho L_{u}, & M^{(5,5)}=\frac{8}{15} \rho L_{u}, & M^{(6,6)}=\frac{8}{15} \rho L_{u}, \\
M^{(1,9)}=-\frac{1}{30} \rho L_{u}, & M^{(2,10)}=-\frac{1}{30} \rho L_{u}, & M^{(5,9)}=\frac{1}{15} \rho L_{u}, \\
M^{(6,10)}=\frac{1}{15} \rho L_{u}, & M^{(9,9)}=\frac{2}{15} \rho L_{u}, & M^{(10,10)}=\frac{2}{15} \rho L_{u}
\end{array}
$$




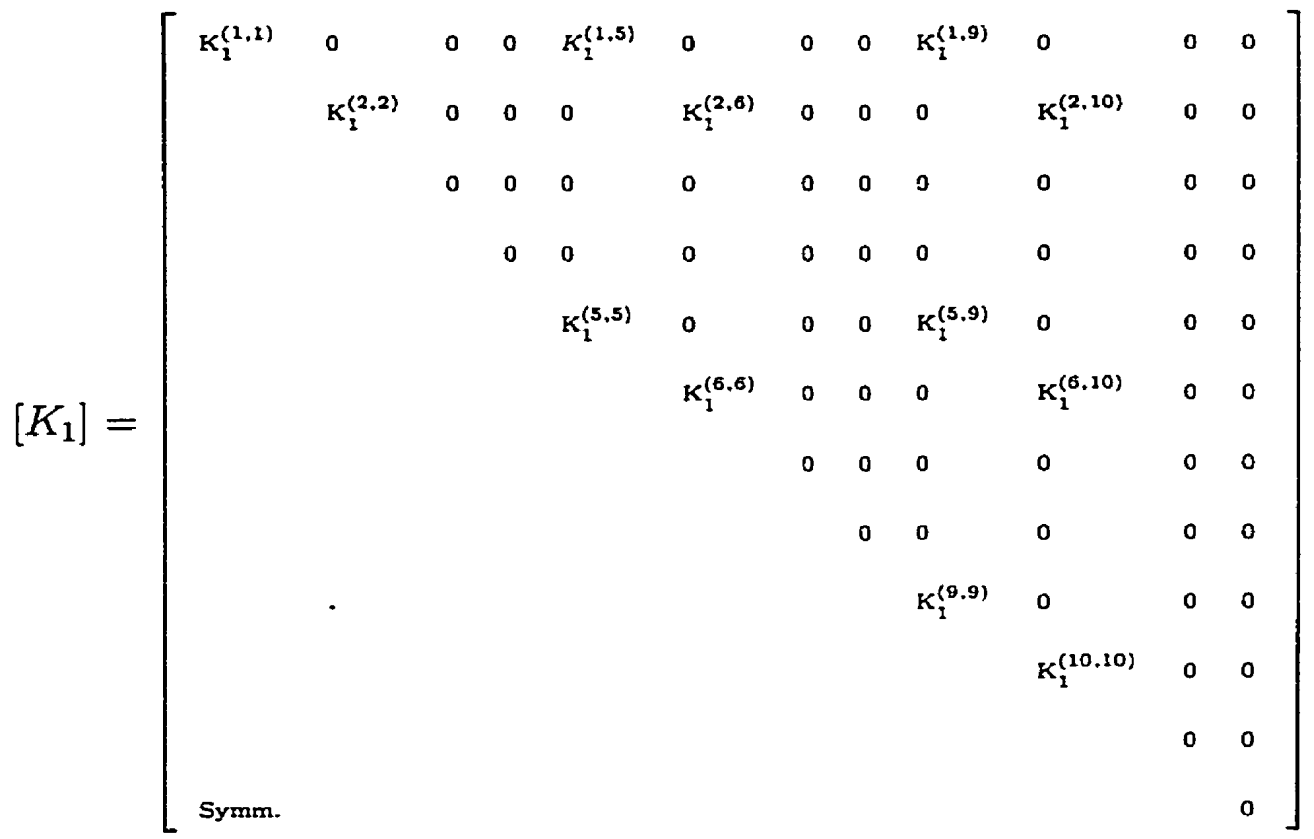

\section{where}

$$
\begin{array}{lll}
K_{1}^{(1,1)}=\frac{2}{15} L_{u} D_{11}^{u}, & K_{1}^{(2,2)}=\frac{2}{15} L_{u} D_{66}^{u}, & K_{1}^{(1,5)}=\frac{1}{15} L_{u} D_{11}^{u}, \\
K_{1}^{(2,6)}=\frac{1}{15} L_{u} D_{66}^{u}, & K_{1}^{(5,5)}=\frac{8}{15} L_{u} D_{11}^{u}, & K_{1}^{(6,6)}=\frac{8}{15} L_{u} D_{66}^{u}, \\
K_{1}^{(1,9)}=-\frac{1}{30} L_{u} D_{11}^{u}, & K_{1}^{(2,10)}=-\frac{1}{30} L_{u} D_{66}^{u}, & K_{1}^{(5,9)}=\frac{1}{15} L_{u} D_{11}^{u}, \\
K_{1}^{(6,10)}=\frac{1}{15} L_{u} D_{66}^{u}, & K_{1}^{(9,9)}=\frac{2}{15} L_{u} D_{11}^{u}, & K_{1}^{(10,10)}=\frac{2}{15} L_{u} D_{66}^{u} .
\end{array}
$$




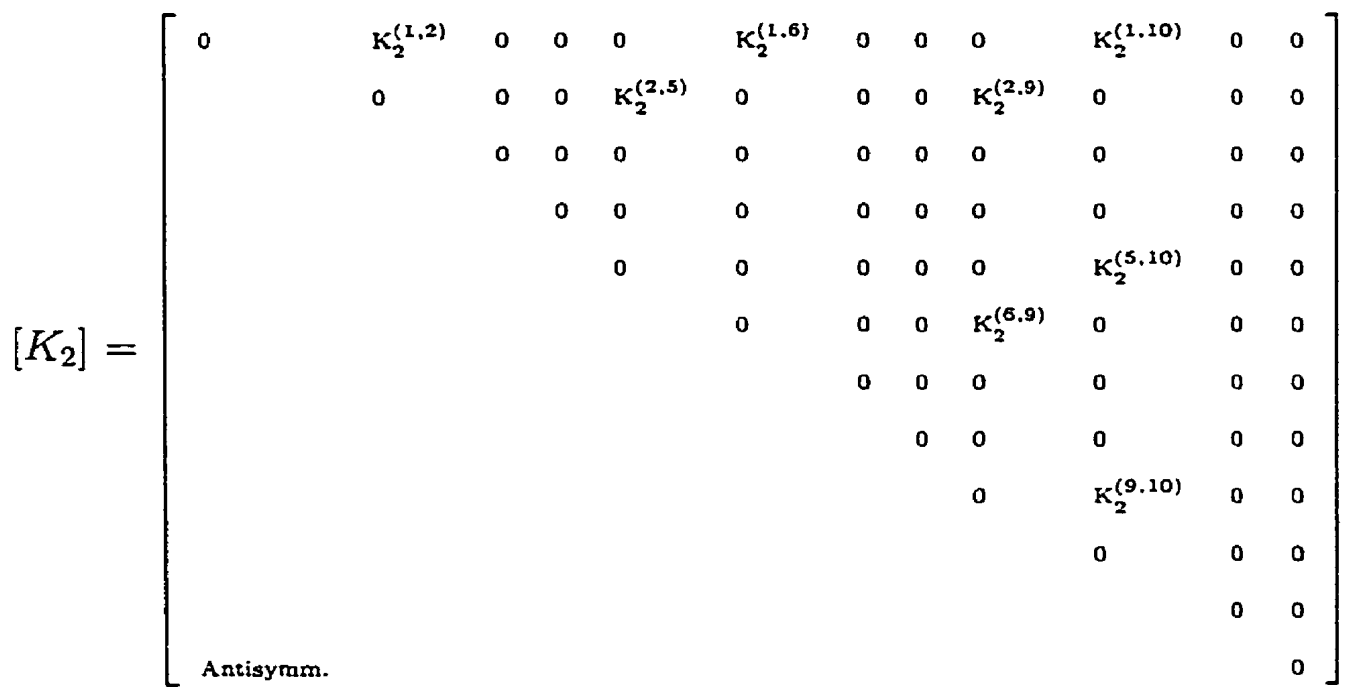

where

$$
\begin{array}{ll}
K_{2}^{(1,2)}=-\frac{1}{2}\left(D_{12}^{u}-D_{66}^{u}\right), & K_{2}^{(1,6)}=\frac{2}{3}\left(D_{12}^{u}+D_{66}^{u}\right) \\
K_{2}^{(2,5)}=\frac{2}{3}\left(D_{12}^{u}+D_{66}^{u}\right), & K_{2}^{(1,10)}=-\frac{1}{6}\left(D_{12}^{u}+D_{66}^{u}\right), \\
K_{2}^{(2,9)}=-\frac{1}{6}\left(D_{12}^{u}+D_{66}^{u}\right), & K_{2}^{(5,10)}=\frac{2}{3}\left(D_{12}^{u}+D_{66}^{u}\right), \\
K_{2}^{(6,9)}=\frac{2}{3}\left(D_{12}^{u}+D_{66}^{u}\right), & K_{2}^{(9,10)}=\frac{1}{2}\left(D_{12}^{u}-D_{66}^{u}\right) .
\end{array}
$$

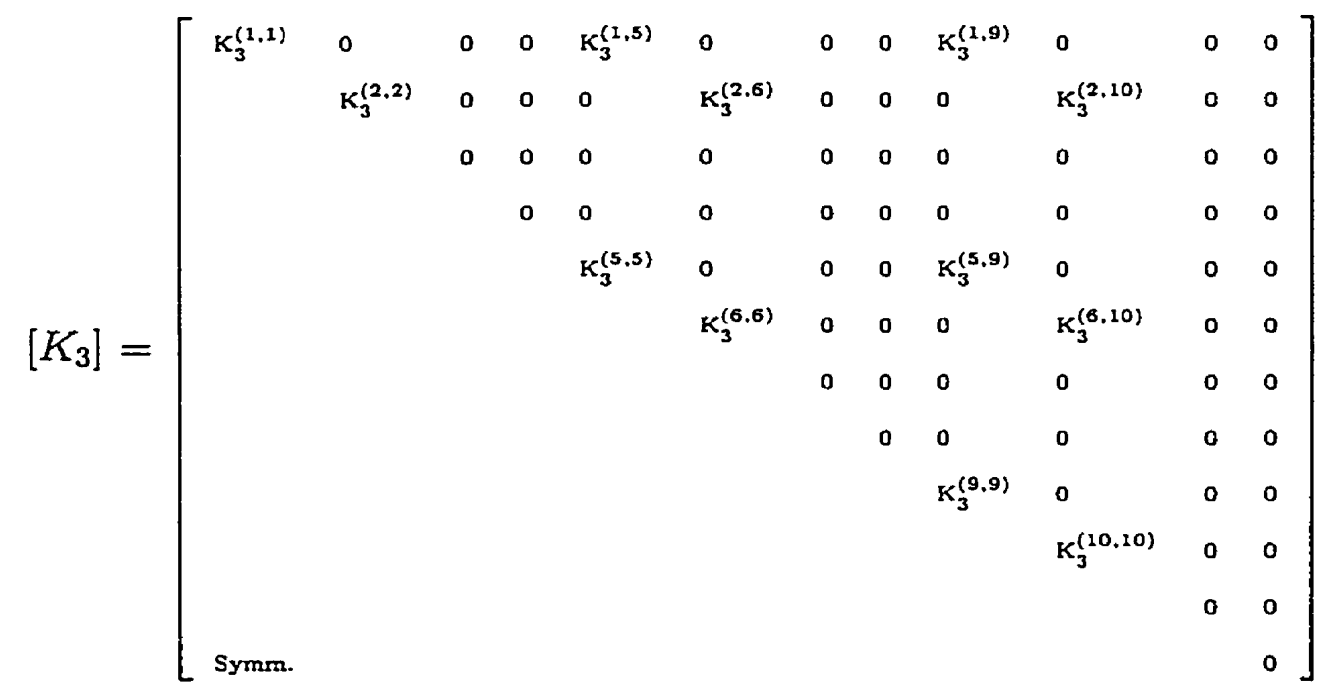

where

$$
\begin{array}{lll}
K_{3}^{(1,1)}=\frac{7}{3 L_{u}} D_{66}^{u}, & K_{3}^{(2,2)}=\frac{7}{3 L_{u}} D_{22}^{u}, & K_{3}^{(1,5)}=-\frac{8}{3 L_{u}} D_{66}^{u} \\
K_{3}^{(2,6)}=-\frac{8}{3 L_{u}} D_{22}^{u}, & K_{3}^{(5,5)}=\frac{16}{3 L_{u}} D_{66}^{u}, & K_{3}^{(6,6)}=\frac{16}{3 L_{u}} D_{22}^{u} \\
K_{3}^{(1,9)}=\frac{1}{3 L_{u}} D_{66}^{u}, & K_{3}^{(2,10)}=\frac{1}{3 L_{u}} D_{22}^{u}, & K_{3}^{(5,9)}=-\frac{8}{3 L_{u}} D_{66}^{u} \\
K_{3}^{(6,10)}=-\frac{8}{3 L_{u}} D_{22}^{u}, & K_{3}^{(9,9)}=\frac{7}{3 L_{u}} D_{66}^{u}, & K_{3}^{(10,10)}=\frac{7}{3 L_{u}} D_{22}^{u}
\end{array}
$$




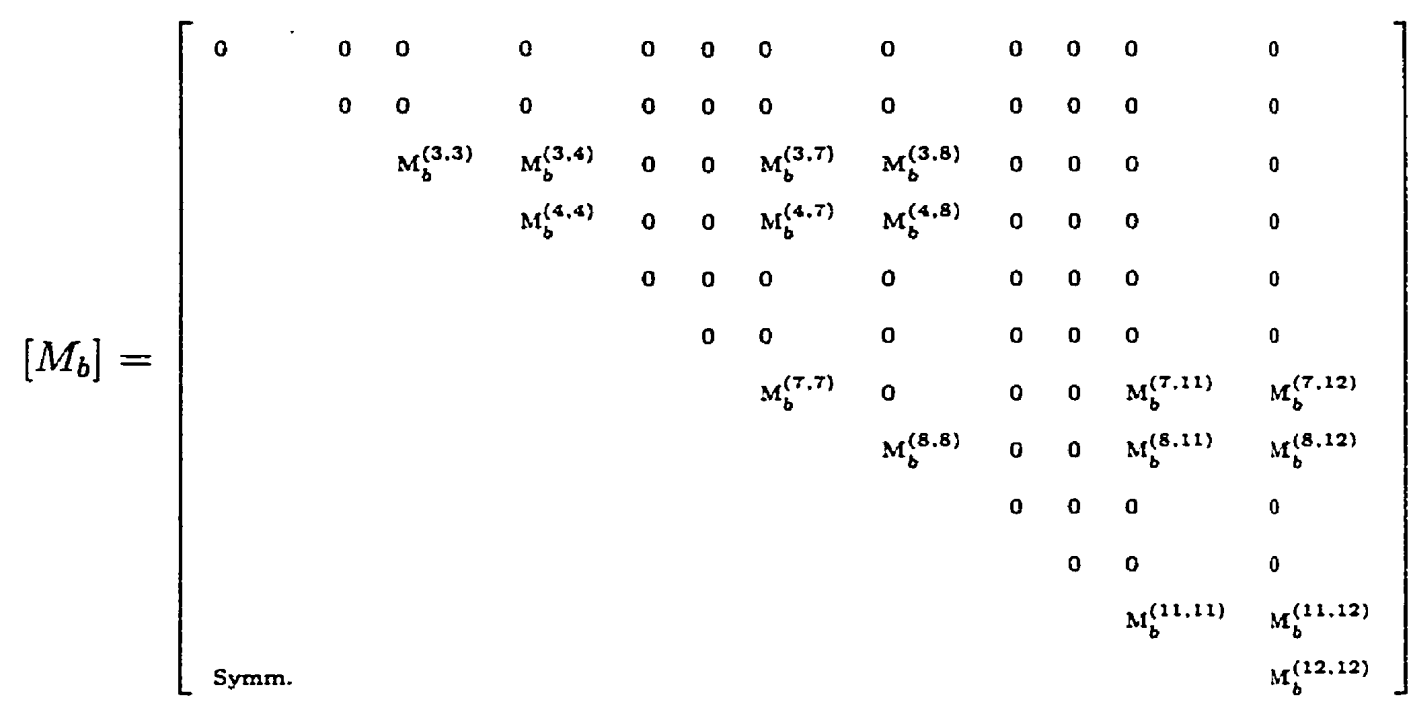

where

$$
\begin{aligned}
& M_{b}^{(3,3)}=\frac{13}{35} \rho L_{w}, \quad M_{b}^{(3,4)}=\frac{11}{210} \rho L_{w}^{2}, \quad M_{b}^{(4,4)}=\frac{1}{105} \rho L_{w}^{3}, \\
& M_{b}^{(3,7)}=\frac{9}{70} \rho L_{w}, \quad M_{b}^{(3,8)}=-\frac{13}{420} \rho L_{w}^{2}: \quad M_{b}^{(4,7)}=\frac{13}{420} \rho L_{w}^{2}, \\
& M_{b}^{(4,8)}=-\frac{1}{140} \rho L_{w}^{3}, \quad M_{b}^{(7,7)}=\frac{26}{35} \rho L_{w}, \quad M_{b}^{(8,8)}=\frac{2}{105} \rho L_{w}^{3} \text {, } \\
& M_{b}^{(7,11)}=\frac{9}{70} \rho L_{w}, \quad M_{b}^{(7,12)}=-\frac{13}{420} \rho L_{w}^{2}, \quad M_{b}^{(8,11)}=\frac{13}{420} \rho L_{w}^{2} \text {, } \\
& M_{b}^{(8,12)}=-\frac{1}{140} \rho L_{w}^{3}, \quad M_{b}^{(11,11)}=\frac{13}{35} \rho L_{w}, \quad M_{b}^{(11,12)}=-\frac{11}{210} \rho L_{w}^{2} \text {, } \\
& M_{b}^{(12,12)}=\frac{1}{105} \rho L_{w}^{3} \text {. }
\end{aligned}
$$

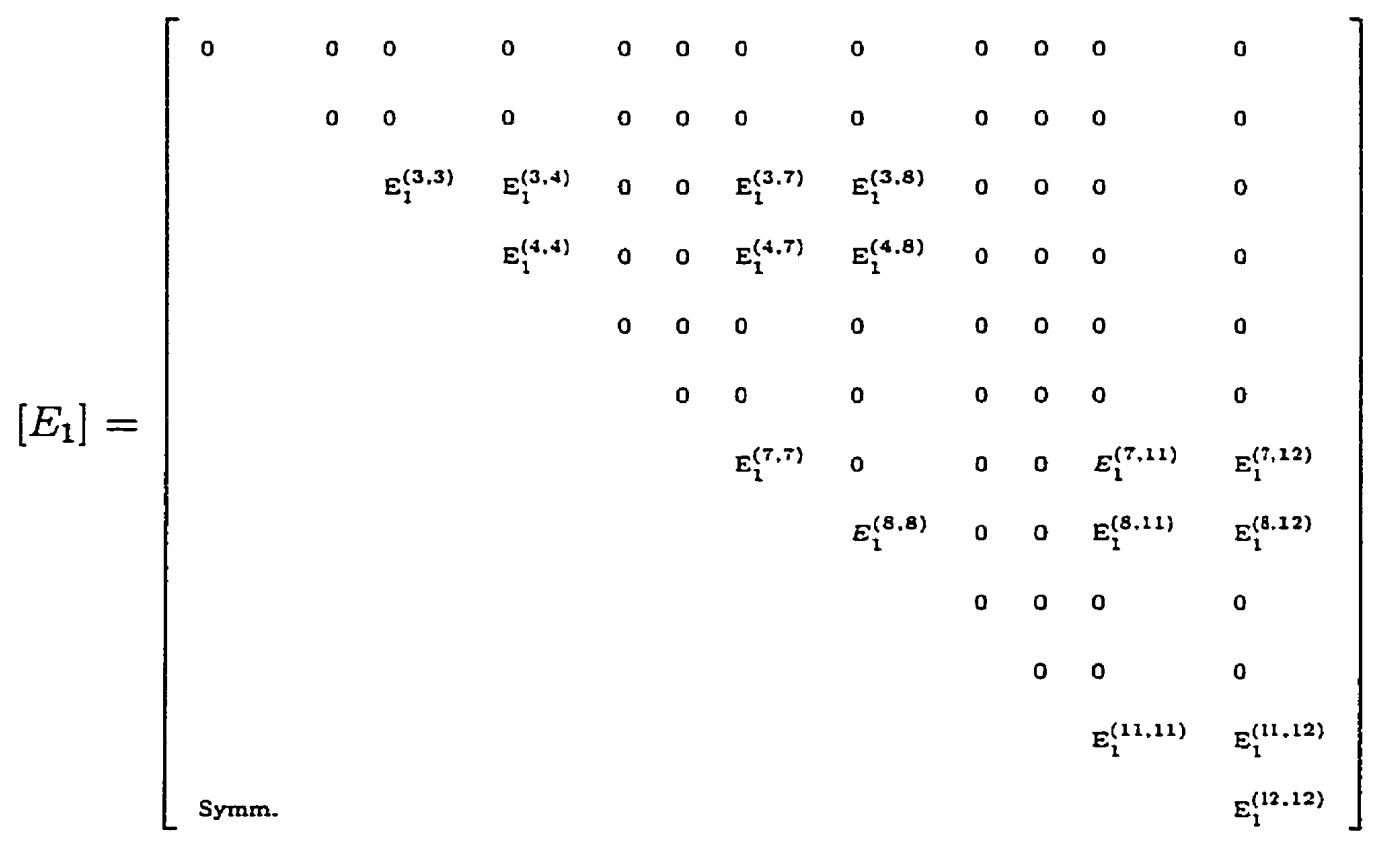




\section{where}

$$
\begin{array}{lll}
E_{1}^{(3,3)}=\frac{13}{35} D_{11}^{w} L_{w}, & E_{1}^{(3,4)}=\frac{11}{210} D_{11}^{w} L_{w}^{2}, & E_{1}^{(4,4)}=\frac{1}{105} D_{11}^{w} L_{w}^{3}, \\
E_{1}^{(3,7)}=\frac{9}{70} D_{11}^{w} L_{w}, & E_{1}^{(3,8)}=-\frac{13}{420} D_{11}^{w} L_{w}^{2}, & E_{1}^{(4,7)}=\frac{13}{420} D_{11}^{w} L_{w}^{2}, \\
E_{1}^{(4,8)}=-\frac{1}{140} D_{11}^{w} L_{w}^{3}, & E_{1}^{(7,7)}=\frac{26}{35} D_{11}^{w} L_{w}, & E_{1}^{(8,8)}=\frac{2}{105} D_{11}^{w} L_{w}^{3}, \\
E_{1}^{(7,11)}=\frac{9}{70} D_{11}^{w} L_{w}, & E_{1}^{(7,12)}=-\frac{13}{420} D_{11}^{w} L_{w}^{2}, & E_{1}^{(8,11)}=\frac{13}{420} D_{11}^{w} L_{w}^{2}, \\
E_{1}^{(8,12)}=-\frac{1}{140} D_{11}^{w} L_{w}^{3}, & E_{1}^{(11,11)}=\frac{13}{35} D_{11}^{w} L_{w}, & E_{1}^{(11,12)}=-\frac{11}{210} D_{11}^{w} L_{w}^{2}, \\
E_{1}^{(12,12)}=\frac{1}{105} D_{11}^{w} L_{w}^{3} . &
\end{array}
$$

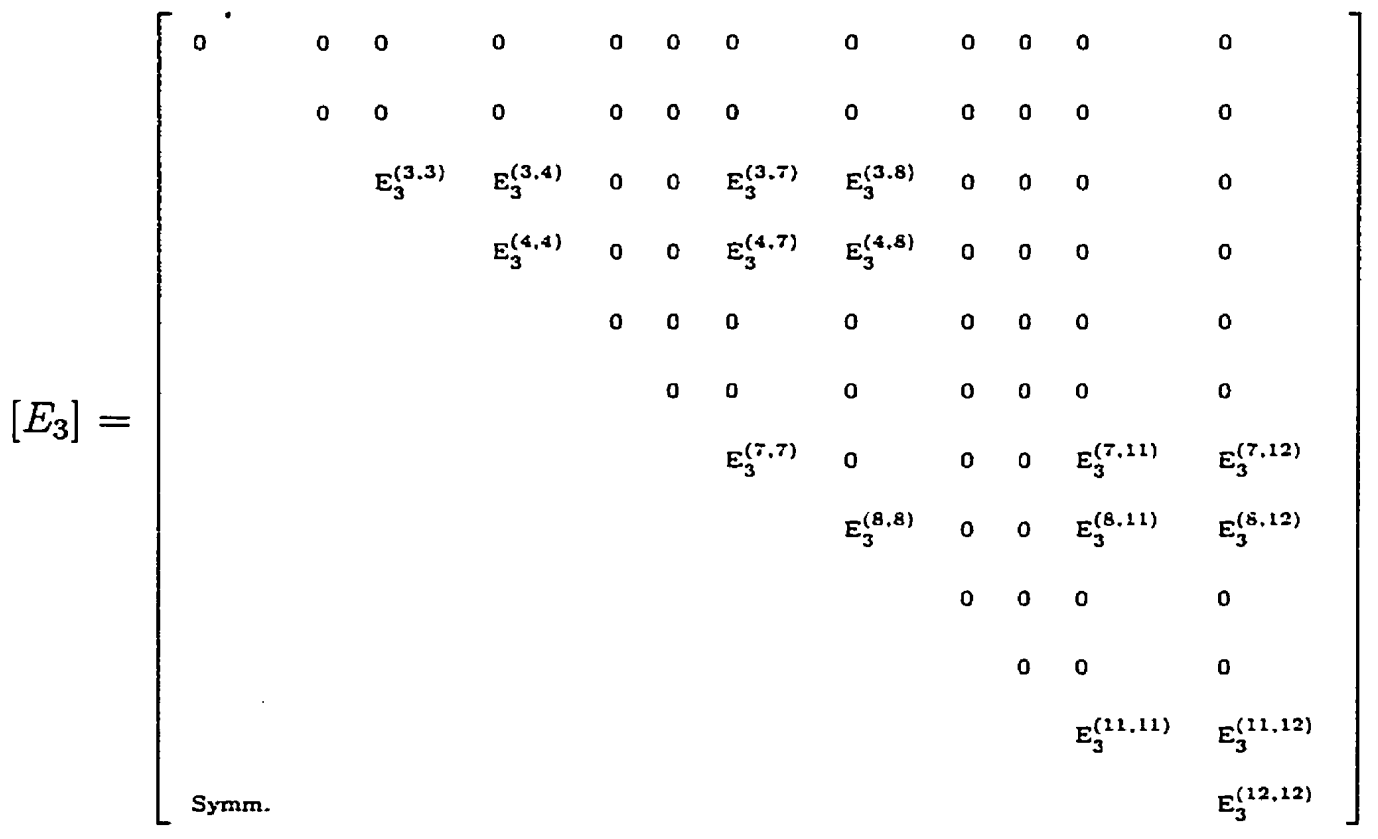

where

$$
\begin{array}{ll}
E_{3}^{(3,3)}=-\frac{12}{5 L_{w}}\left(D_{12}^{w}+2 D_{66}^{w}\right), & E_{3}^{(3,4)}=-\frac{6}{5} D_{12}^{w}-\frac{2}{5} D_{66}^{w}, \\
E_{3}^{(4,4)}=-\frac{4}{15} L_{w}\left(D_{12}^{w}+2 D_{66}^{w}\right), & E_{3}^{(3,7)}=\frac{12}{5 L_{w}}\left(D_{12}^{w}+2 D_{66}^{w}\right), \\
E_{3}^{(3,8)}=-\frac{1}{5} D_{12}^{w}-\frac{2}{5} D_{66}^{w}, & E_{3}^{(4,7)}=\frac{1}{5} D_{12}^{w}+\frac{2}{5} D_{66}^{w}, \\
E_{3}^{(4,8)}=\frac{1}{15} L_{w}\left(D_{12}^{w}+2 D_{66}^{w}\right), & E_{3}^{(7,7)}=-\frac{24}{5 L_{w}}\left(D_{12}^{w}+2 D_{66}^{w}\right) \\
E_{3}^{(8,8)}=-\frac{8}{15} L_{w}\left(D_{12}^{w}+2 D_{66}^{w}\right), & E_{3}^{(7,11)}=\frac{12}{5 L_{w}}\left(D_{12}^{w}+2 D_{66}^{w}\right), \\
E_{3}^{(7,12)}=-\frac{1}{5} D_{12}^{w}-\frac{2}{5} D_{66}^{w}, & E_{3}^{(8,11)}=\frac{1}{5} D_{12}^{w}+\frac{2}{5} D_{66}^{w}, \\
E_{3}^{(8,12)}=\frac{1}{15} L_{w}\left(D_{12}^{w}+2 D_{66}^{w}\right), & E_{3}^{(11,11)}=-\frac{12}{5 L_{w}}\left(D_{12}^{w}+2 D_{66}^{w}\right) \\
E_{3}^{(11,12)}=\frac{6}{5} D_{12}^{w}+\frac{2}{5} D_{66}^{w}, & E_{3}^{(12,12)}=-\frac{4}{15} L_{w}\left(D_{12}^{w}+2 D_{66}^{w}\right) .
\end{array}
$$


APPENDIX A. MATRICES OF THE 2D WAVE PROPAGATION PROBLEM 59

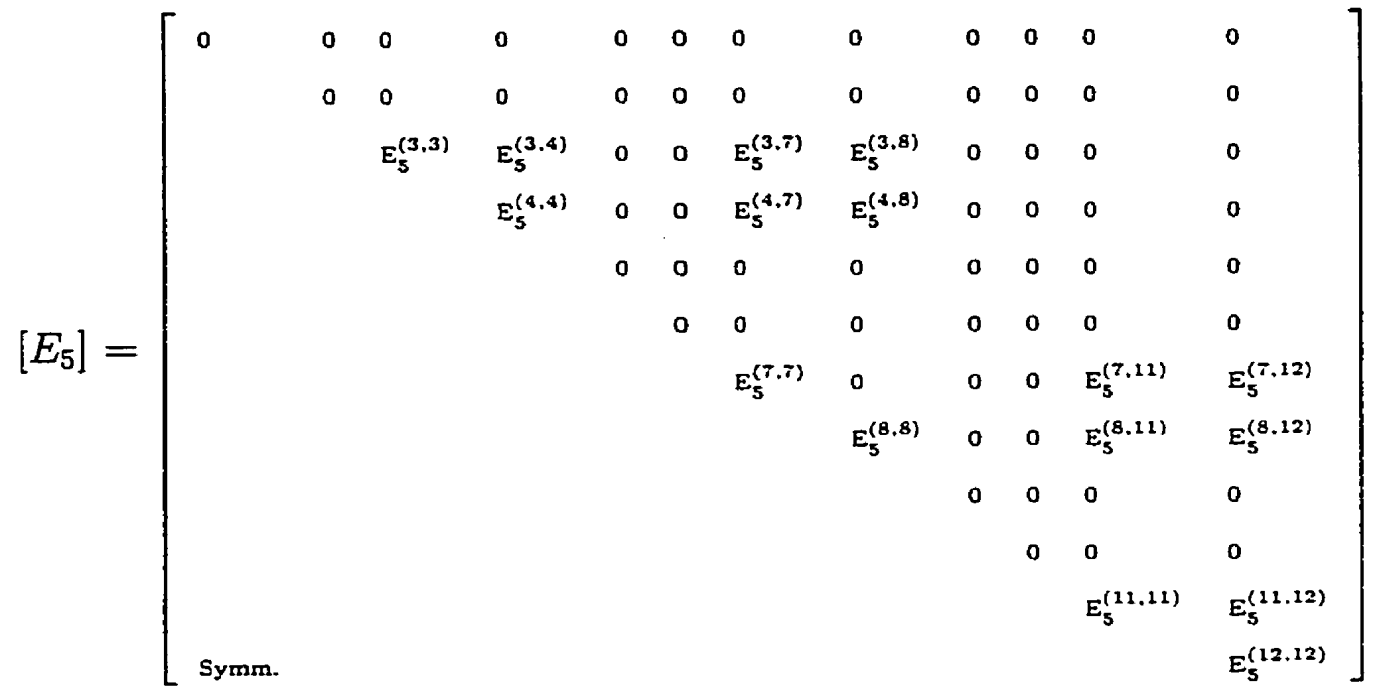

where

$$
\begin{array}{lll}
E_{5}^{(3,3)}=12 \frac{D_{22}^{w}}{L_{w}^{3}}, & E_{5}^{(3,4)}=6 \frac{D_{22}^{w}}{L_{w}^{2}}, & E_{5}^{(4,4)}=4 \frac{D_{22}^{w}}{L_{w}^{w}}, \\
E_{5}^{(3,7)}=-12 \frac{D_{22}^{w}}{L_{w}^{3}}, & E_{5}^{(3,8)}=6 \frac{D_{22}^{w}}{L_{W_{w 2}^{2}}^{2}}, & E_{5}^{(4,7)}=-6 \frac{D_{22}^{w}}{L_{w}^{2}}, \\
E_{5}^{(4,8)}=2 \frac{D_{22}^{w}}{L_{w}}, & E_{5}^{(7,7)}=24 \frac{D_{22}^{w}}{L^{3}}, & E_{5}^{(8,8)}=8 \frac{D_{22}^{w}}{L_{w}^{w}}, \\
E_{5}^{(7,11)}=-12 \frac{D_{22}^{w}}{L_{w}^{3}}, & E_{5}^{(7,12)}=6 \frac{D_{22}^{w}}{L_{w}^{2}}, & E_{5}^{(8,11)}=-6 \frac{D_{22}^{w}}{L_{w_{w}^{w}}^{2}}, \\
E_{5}^{(8,12)}=2 \frac{D_{22}^{w}}{L_{w}^{w}}, & E_{5}^{(11,11)}=12 \frac{D_{22}^{w}}{L_{w}^{3}}, & E_{5}^{(11,12)}=-6 \frac{D_{22}^{2}}{L_{w}^{2}}, \\
E_{5}^{(12,12)}=4 \frac{D_{22}^{w}}{L_{w}} . &
\end{array}
$$




\section{Appendix B}

\section{D RR Vibration of Simply Supported Members}

\section{B.1 Introduction}

In this section of the study the element equations created earlier using the twodimensional Rayleigh-Ritz approximation will be modified from their original form to model the vibration of thin-walled structural members simply supported in the longitudinal direction at their ends.

\section{B.2 Formulation}

This problem is an extension of the two-dimensional wave propagation problem derived earlier. In the wave propagation problem it is assumed that the length of the member is infinite in the $\mathrm{x}=\mathrm{X}$ direction. To model simple supports at the ends of the beam there are only a few minor modifications. For simple supports it is necessary that the displacements $u(0) \neq 0, u(a) \neq 0, v(0)=v(a)=w(0)=w(a)=\theta(0)=$ $\theta(a)=0$ where $\theta=\frac{\partial w}{\partial x}$. The vibration is assumed to be sinusoidal therefore

$$
\begin{aligned}
& u(x)=\cos \lambda x \\
& v(x)=\sin \lambda x \\
& w(x)=\sin \lambda x \\
& \theta(x)=\sin \lambda x
\end{aligned}
$$


where $\lambda=\frac{m \pi}{a}$, and $a$ is the length between supports and $m$ is the number of half wave lengths, i.e. $m=1,2,3 \ldots$.

Thus we assume that the nodal displacements as a function of $x$ and $t$ are

$$
q_{u}(x, t)=\left\{\begin{array}{l}
u_{1}(x, t) \\
u_{2}(x, t) \\
u_{3}(x, t)
\end{array}\right\}=\left\{Q_{0}^{u}\right\} e^{+i \omega t} \cos \lambda x
$$

and

$$
q_{\alpha}(x, t)=\left\{\begin{array}{l}
\alpha_{1}(x, t) \\
\alpha_{2}(x, t) \\
\alpha_{3}(x, t)
\end{array}\right\}=\left\{Q_{0}^{\alpha}\right\} e^{+i \omega t} \sin \lambda x
$$

where $\alpha=v, w, \theta$.

We now return to the element equations, Equations 2.14 and 2.35, derived earlier using plane stress analysis. However, due to changes in the order of the displacement field the rows and columns of the elemental matrices $[M],\left[K_{1}\right],\left[K_{2}\right],\left[K_{3}\right],\left[M_{b}\right],\left[E_{1}\right]$, $\left[E_{3}\right]$ and $\left[E_{5}\right]$ must also be changed accordingly. The revised matrices are shown in Appendix C.

Substituting Equations B.5 and B.6 into Equation 2.14 results in:

$$
\begin{array}{r}
+\omega^{2}[M]\left\{\begin{array}{c}
\cos \lambda x\left\{Q_{0}^{u}\right\} \\
\sin \lambda x\left\{Q_{0}^{v}\right\} \\
\sin \lambda x\left\{Q_{0}^{w}\right\} \\
\sin \lambda x\left\{Q_{0}^{\theta}\right\}
\end{array}\right\}+\left[K_{1}\right]\left\{\begin{array}{c}
-\lambda^{2} \cos \lambda x\left\{Q_{0}^{u}\right\} \\
-\lambda^{2} \sin \lambda x\left\{Q_{0}^{v}\right\} \\
-\lambda^{2} \sin \lambda x\left\{Q_{0}^{w}\right\} \\
-\lambda^{2} \sin \lambda x\left\{Q_{0}^{\theta}\right\}
\end{array}\right\} \\
+\left[K_{2}\right]\left\{\begin{array}{c}
-\lambda \sin \lambda x\left\{Q_{0}^{u}\right\} \\
\lambda \cos \lambda x\left\{Q_{0}^{v}\right\} \\
\lambda \cos \lambda x\left\{Q_{0}^{w}\right\} \\
\lambda \cos \lambda x\left\{Q_{0}^{\theta}\right\}
\end{array}\right\}-\left[K_{3}\right]\left\{\begin{array}{c}
\cos \lambda x\left\{Q_{0}^{u}\right\} \\
\sin \lambda x\left\{Q_{0}^{v}\right\} \\
\sin \lambda x\left\{Q_{0}^{w}\right\} \\
\sin \lambda x\left\{Q_{0}^{\theta}\right\}
\end{array}\right\}=\left\{\begin{array}{l}
\{0\} \\
\{0\} \\
\{0\} \\
\{0\}
\end{array}\right\}
\end{array}
$$

Each of the matrices of Equation B.7 can be expressed in terms of zero and nonzero submatrices as shown below:

$$
\begin{gathered}
{[M]=\left[\begin{array}{cc}
{\left[M_{u}\right]} & 0 \\
0 & {\left[M_{v}\right]}
\end{array}\right]} \\
{\left[K_{1}\right]=\left[\begin{array}{cc}
{\left[K_{1 u}\right]} & 0 \\
0 & {\left[K_{1 v}\right]}
\end{array}\right]}
\end{gathered}
$$




$$
\begin{aligned}
& {\left[K_{2}\right]=\left[\begin{array}{cc}
0 & {\left[K_{2 v}\right]} \\
{\left[K_{2 u}\right]} & 0
\end{array}\right]} \\
& {\left[K_{3}\right]=\left[\begin{array}{cc}
{\left[K_{3 u}\right]} & 0 \\
0 & {\left[K_{3 v}\right]}
\end{array}\right] .}
\end{aligned}
$$

Submatrices $\left[M_{u}\right],\left[M_{v}\right],\left[K_{1 u}\right],\left[K_{1 v}\right],\left[K_{2 u}\right],\left[K_{2 v}\right],\left[K_{3 u}\right]$ and $\left[K_{3 v}\right]$ are defined in Appendix C.

Expanding Equation B.7 results in Equations B.12 and B.13:

$$
\begin{aligned}
& +\omega^{2}\left[M_{u}\right]\left\{Q_{0}^{u}\right\}-\lambda^{2}\left[K_{1 u}\right]\left\{Q_{0}^{u}\right\}+\lambda\left[K_{2 v}\right]\left\{Q_{0}^{v}\right\}-\left[K_{3}\right]\left\{Q_{0}^{u}\right\}=\{0\} \\
& +\omega^{2}\left[M_{v}\right]\left\{Q_{0}^{u}\right\}-\lambda^{2}\left[K_{1 v}\right]\left\{Q_{0}^{v}\right\}-\lambda\left[K_{2 u}\right]\left\{Q_{0}^{u}\right\}-\left[K_{3}\right]\left\{Q_{0}^{v}\right\}=\{0\} .
\end{aligned}
$$

Similarly substituting Equations B.5 and B.6 into Equation 2.35 results in:

$$
+\omega^{2}\left[M_{\alpha}\right]\left\{Q_{0}^{\alpha}\right\}-\lambda^{4}\left[E_{1 \alpha}\right]\left\{Q_{0}^{\alpha}\right\}+\lambda^{2}\left[E_{3 \alpha}\right]\left\{Q_{0}^{\alpha}\right\}-\left[E_{5 \alpha}\right]\left\{Q_{0}^{\alpha}\right\}=\{0\}
$$

where

$$
\left\{Q_{0}^{\alpha}\right\}=\left\{\begin{array}{l}
\left\{Q_{0}^{w}\right\} \\
\left\{Q_{0}^{\theta}\right\}
\end{array}\right\}
$$

Submatrices $\left[M_{b \alpha}\right],\left[E_{1 \alpha}\right],\left[E_{3 \alpha}\right]$ and $\left[E_{5 \alpha}\right]$ are defined in Appendix $C$.

Arranging the three equations into a convenient matrix form gives

$$
\left[\begin{array}{cccc}
{[1]} & {[2]} & 0 & 0 \\
{[3]} & {[4]} & 0 & 0 \\
0 & 0 & & {[5]}
\end{array}\right]\left\{\begin{array}{l}
\left\{Q_{0}^{u}\right\} \\
\left\{Q_{0}^{v}\right\} \\
\left\{Q_{0}^{w}\right\} \\
\left\{Q_{0}^{\theta}\right\}
\end{array}\right\}=\{0\}
$$

where

$$
\begin{aligned}
& {[1]_{3 * 3}=+\omega^{2}\left[M_{u}\right]-\lambda^{2}\left[K_{1 u}\right]-\left[K_{3 u}\right]} \\
& {[2]_{3 * 3}=+\lambda\left[K_{2 v}\right]} \\
& {[3]_{3 * 3}=-\lambda\left[K_{2 u}\right]} \\
& {[4]_{3 * 3}=+\omega^{2}\left[M_{v}\right]-\lambda^{2}\left[K_{1 v}\right]-\left[K_{3 v}\right]} \\
& {[5]_{6 * 6}=+\omega^{2}\left[M_{\alpha}\right]-\lambda^{4}\left[E_{1 \alpha}\right]+\lambda^{2}\left[E_{3 \alpha}\right]-\left[E_{5 \alpha}\right]}
\end{aligned}
$$


The next step is to transform the local element equations into the global coordinate system. The transformation matrix $[T]$ is used to transform the element equations from the local to global coordinate system. Resulting in the global element equations, Equation B.17, in the form $\left[K_{g}\right]\left\{Q_{0}^{g}\right\}=\{0\}$.

$$
[T]^{T}\left[\begin{array}{ccccc}
{[1]} & {[2]} & - & - \\
{[3]} & {[4]} & - & - \\
- & - & & {[5]}
\end{array}\right][T]\left\{\begin{array}{c}
\left\{Q_{0}^{u g}\right\} \\
\left\{Q_{0}^{v g}\right\} \\
\left\{Q_{0}^{w g}\right\} \\
\left\{Q_{0}^{\theta g}\right\}
\end{array}\right\}=\{0\}
$$

The elements of the transformation matrix $[T]$, in Equation B.17 are:

$$
[T]=\left[\begin{array}{cccccccccccc}
1 & 0 & 0 & 0 & 0 & 0 & 0 & 0 & 0 & 0 & 0 & 0 \\
0 & 1 & 0 & 0 & 0 & 0 & 0 & 0 & 0 & 0 & 0 & 0 \\
0 & 0 & 1 & 0 & 0 & 0 & 0 & 0 & 0 & 0 & 0 & 0 \\
0 & 0 & 0 & C & 0 & S & 0 & 0 & 0 & 0 & 0 & 0 \\
0 & 0 & 0 & 0 & C & 0 & S & 0 & 0 & 0 & 0 & 0 \\
0 & 0 & 0 & 0 & 0 & C & 0 & S & 0 & 0 & 0 & 0 \\
0 & 0 & 0 & -S & 0 & 0 & C & 0 & 0 & 0 & 0 & 0 \\
0 & 0 & 0 & 0 & -S & 0 & 0 & C & 0 & 0 & 0 & 0 \\
0 & 0 & 0 & 0 & 0 & -S & 0 & 0 & C & 0 & 0 & 0 \\
0 & 0 & 0 & 0 & 0 & 0 & 0 & 0 & 0 & 1 & 0 & 0 \\
0 & 0 & 0 & 0 & 0 & 0 & 0 & 0 & 0 & 0 & 1 & 0 \\
0 & 0 & 0 & 0 & 0 & 0 & 0 & 0 & 0 & 0 & 0 & 1
\end{array}\right]
$$

where $S=\sin (\theta)$ and $C=\cos (\theta)$.

\section{B.3 Eigenvalue Problem}

The global element equations, Equation B.17, are then assembled to obtain the global structure equations, Equation B.18, in the form $[\mathbf{K}]\left\{\mathbf{Q}_{0}^{\mathbf{g}}\right\}=\{\mathbf{0}\}$

$$
\left[\begin{array}{cccc}
{[1]} & {[2]} & 0 & 0 \\
{[3]} & {[4]} & 0 & 0 \\
0 & 0 & & {[\mathbf{5}]}
\end{array}\right]\left\{\begin{array}{c}
\left\{\mathbf{Q}_{0}^{\mathbf{u g}}\right\} \\
\left\{\mathbf{Q}_{0}^{\mathbf{v g}}\right\} \\
\left\{\mathbf{Q}_{0}^{\mathbf{w g}}\right\} \\
\left\{\mathbf{Q}_{0}^{\theta \mathbf{g}}\right\}
\end{array}\right\}=\{0\}
$$


We can now arrange Equation B.18 into the eigenvalue problem given below

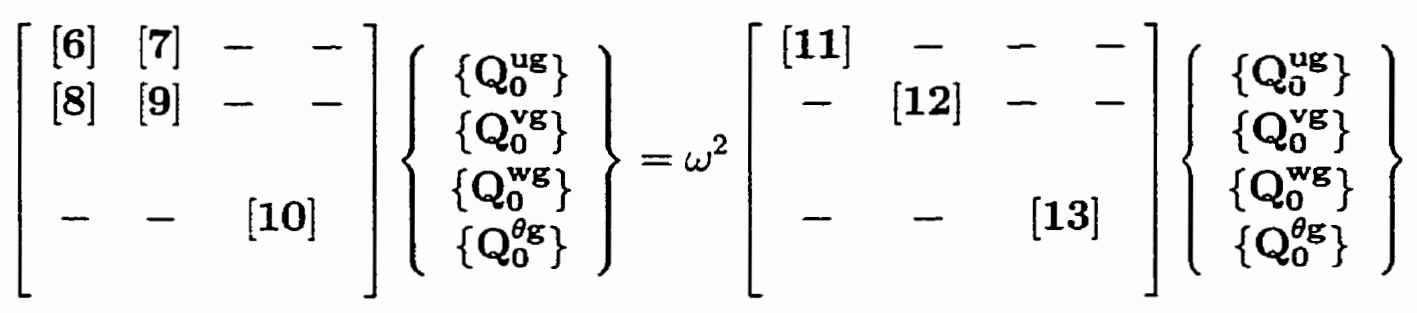

where

$$
\begin{aligned}
{[6]_{3 * 3} } & =-\lambda^{2}\left[K_{1 u}\right]-\left[K_{3 u}\right] \\
{[7]_{3 * 3} } & =+\lambda\left[K_{2 v}\right] \\
{[8]_{3 * 3} } & =-\lambda\left[K_{2 u}\right] \\
{[9]_{3 * 3} } & =-\lambda^{2}\left[K_{1 v}\right]-\left[K_{3 v}\right] \\
{[10]_{6 * 6} } & =-\lambda^{4}\left[E_{1}\right]+\lambda^{2}\left[E_{3}\right]-\left[E_{5}\right] \\
{[11]_{3 * 3} } & =-\left[M_{u}\right] \\
{[12]_{3 * 3} } & =-\left[M_{v}\right] \\
{[13]_{6 * 6} } & =-\left[M_{w}\right] .
\end{aligned}
$$

From this eigenvalue problem frequencies, $\omega$ are obtained for a given number of half wavelengths, $m$.

\section{B.4 Summary}

The accuracy of the plane stress model of the vibration of thin-walled structural members simply supported at their ends will be discussed in Chapter 5 . The plane stress approximation will be compared to a similar approximation based on threedimensional theory as well as the simplified analytical free vibration model which will both be presented later in the study. 


\section{Appendix C}

\section{Matrices for 2D RR Vibration}

\section{Problem}

In changing from the wave propagation problem to the vibration problem we must first rearrange the rows of the displacment vector $\{q(x, t)\}$. Instead of

$$
\{q(x, t)\}=\left\{\begin{array}{c}
u_{1}(x, t) \\
v_{1}(x, t) \\
w_{1}(x, t) \\
\theta_{1}(x, t) \\
u_{2}(x, t) \\
v_{2}(x, t) \\
w_{2}(x, t) \\
\theta_{2}(x, t) \\
u_{3}(x, t) \\
v_{3}(x, t) \\
w_{3}(x, t) \\
\theta_{3}(x, t)
\end{array}\right\}
$$


in the wave propagation problem, we now have

$$
\{q(x, t)\}=\left\{\begin{array}{l}
\left\{q_{u}(x, t)\right\} \\
\left\{q_{v}(x, t)\right\} \\
\left\{q_{w}(x, t)\right\} \\
\left\{q_{\theta}(x, t)\right\}
\end{array}\right\}=\left\{\begin{array}{l}
u_{1}(x, t) \\
u_{2}(x, t) \\
u_{3}(x, t) \\
v_{1}(x, t) \\
v_{2}(x, t) \\
v_{3}(x, t) \\
w_{1}(x, t) \\
w_{2}(x, t) \\
w_{3}(x, t) \\
\theta_{1}(x, t) \\
\theta_{2}(x, t) \\
\theta_{3}(x, t)
\end{array}\right\}
$$

for the vibration problem.

The end result is the rows and columns of the element matrices must also be changed. The element matrix $[M]$ for the vibration problem is shown below. The elements of the matrix have been defined in Appendix A.

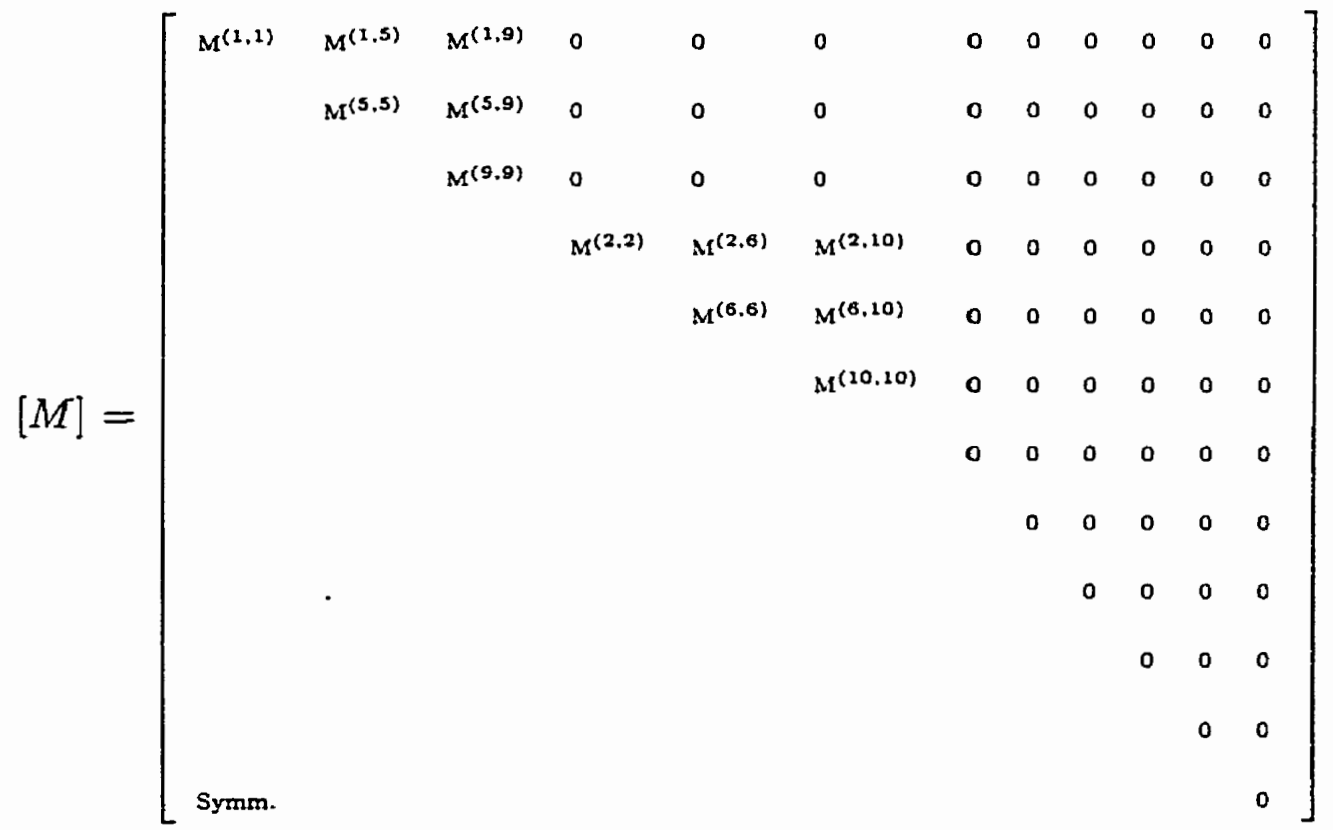

It is here that we note that matrix $[M]$ is comprised of two non-zero submatrices which we will define as: 


$$
\left[M_{u}\right]=\left[\begin{array}{lll}
M^{(1.1)} & M^{(1.5)} & M^{(1,9)} \\
& M^{(5,5)} & M^{(5,9)} \\
\text { Symm. } & & M^{(9.9)}
\end{array}\right] \text { and }\left[M_{v}\right]=\left[\begin{array}{lll}
M^{(2.2)} & M^{(2,8)} & M^{(2,10)} \\
& M^{(6,6)} & M^{(6.10)} \\
\text { Symm. } & & M^{(10.10)}
\end{array}\right]
$$

The element matrix $\left[K_{1}\right]$ for the vibration problem is shown below. The elements of the matrix have been defined in Appendix A.

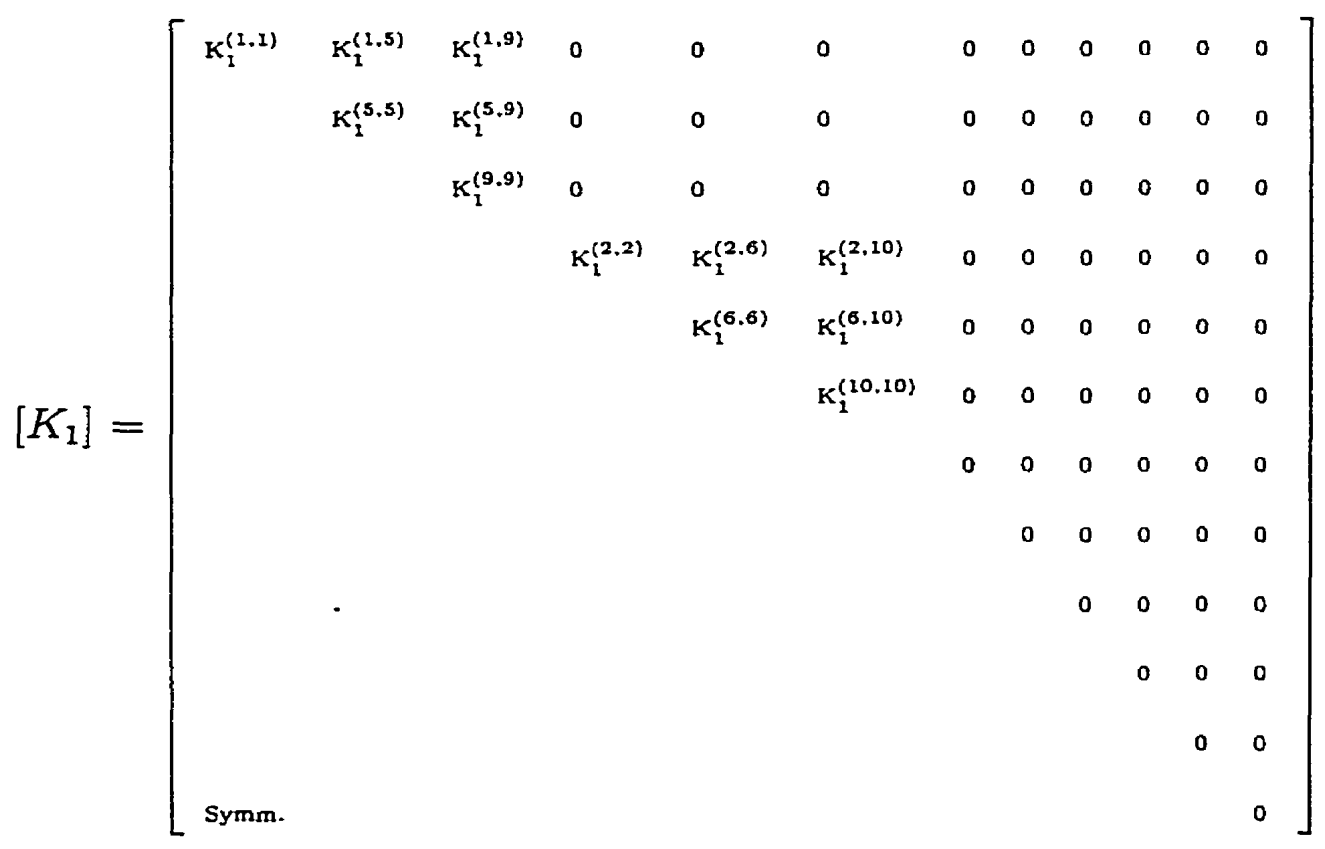

It is here that we note that matrix $K_{1}$ is comprised of two non-zero submatrices which we will define as

$$
\left[K_{1 u}\right]=\left[\begin{array}{lll}
K_{1}^{(1,1)} & K_{1}^{(1,5)} & K_{1}^{(1,9)} \\
& K_{1}^{(5,5)} & K_{1}^{(5,9)} \\
\text { Symm. } & & K_{1}^{(9,9)}
\end{array}\right] \text { and }\left[K_{1 v}\right]=\left[\begin{array}{lll}
K_{1}^{(2,2)} & K_{1}^{(2,6)} & K_{1}^{(2,10)} \\
& K_{1}^{(8,6)} & K_{1}^{(6,10)} \\
\text { symm. } & & K_{1}^{(10,10)}
\end{array}\right]
$$

The element matrix $\left[K_{2}\right]$ for the vibration problem is shown next. The elements of the matrix have been defined in Appendix A. 


$$
\left[K_{2}\right]=\left[\begin{array}{llllllllllll}
0 & 0 & 0 & K_{2}^{(1,2)} & K_{2}^{(1,6)} & K_{2}^{(1,20)} & 0 & 0 & 0 & 0 & 0 & 0 \\
0 & 0 & 0 & -K_{2}^{(2.5)} & 0 & K_{2}^{(5.10)} & 0 & 0 & 0 & 0 & 0 & 0 \\
0 & 0 & 0 & -K_{2}^{(2,9)} & -K_{2}^{(6.9)} & K_{2}^{(9.10)} & 0 & 0 & 0 & 0 & 0 & 0 \\
-K_{2}^{(1,2)} & K_{2}^{(2,5)} & K_{2}^{(2.9)} & 0 & 0 & 0 & 0 & 0 & 0 & 0 & 0 & 0 \\
-K_{2}^{(1,6)} & 0 & K_{2}^{(6.9)} & 0 & 0 & 0 & 0 & 0 & 0 & 0 & 0 & 0 \\
-K_{2}^{(1,10)} & -K_{2}^{(5.10)} & -K_{2}^{(9.10)} & 0 & 0 & 0 & 0 & 0 & 0 & 0 & 0 & 0 \\
0 & 0 & 0 & 0 & 0 & 0 & 0 & 0 & 0 & 0 & 0 & 0 \\
0 & 0 & 0 & 0 & 0 & 0 & 0 & 0 & 0 & 0 & 0 & 0 \\
0 & 0 & 0 & 0 & 0 & 0 & 0 & 0 & 0 & 0 & 0 & 0 \\
0 & 0 & 0 & 0 & 0 & 0 & 0 & 0 & 0 & 0 & 0 & 0 \\
0 & 0 & 0 & 0 & 0 & 0 & 0 & 0 & 0 & 0 & 0 & 0 \\
0 & 0 & 0 & 0 & 0 & 0 & 0 & 0 & 0 & 0 & 0 & 0
\end{array}\right]
$$

Matrix $K_{2}$ is comprised of two non-zero submatrices which we will define as

$$
\left[K_{2 v}\right]=\left[\begin{array}{lll}
K_{2}^{(1.2)} & K_{2}^{(1.6)} & K_{2}^{(1.10)} \\
-K_{2}^{(2.5)} & 0 & K_{2}^{(5.10)} \\
-K_{2}^{(2.9)} & -K_{2}^{(6.9)} & K_{2}^{(9.10)}
\end{array}\right] \text { and }\left[K_{2 u}\right]=\left[\begin{array}{lll}
-K_{2}^{(1.2)} & K_{2}^{(2.5)} & K_{2}^{(2.9)} \\
-K_{2}^{(1.6)} & 0 & K_{2}^{(6.9)} \\
-K_{2}^{(1.10)} & -K_{2}^{(5.10)} & -K_{2}^{(9.10)}
\end{array}\right]
$$

The element matrix $\left[K_{3}\right]$ for the vibration problem is shown below. The elements of the matrix have been defined in Appendix A.

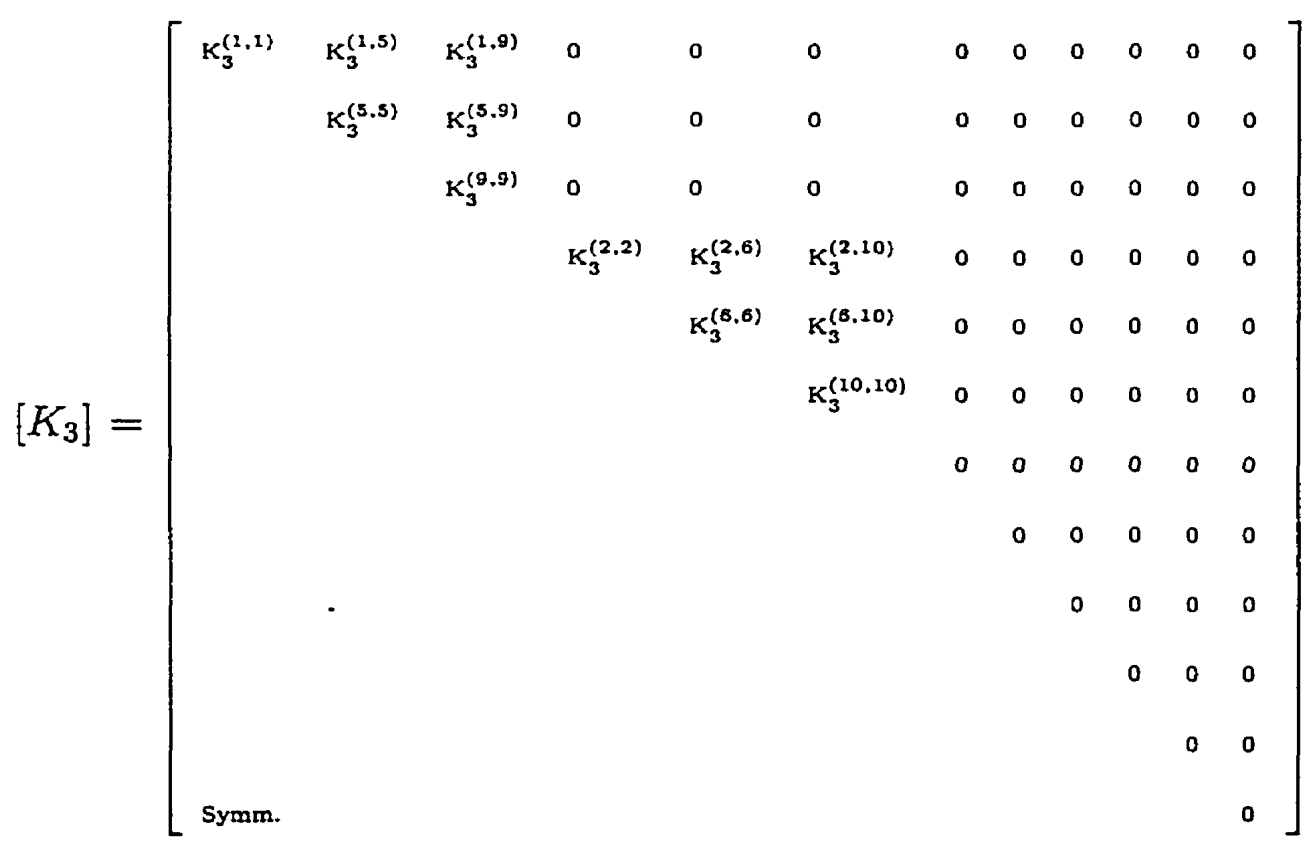


It is here that we note that matrix $K_{3}$ is comprised of two non-zero submatrices which we will define as

$$
\left[K_{3 u}\right]=\left[\begin{array}{lll}
K_{3}^{(1,1)} & K_{3}^{(1,5)} & K_{3}^{(1,9)} \\
& K_{3}^{(5.5)} & K_{3}^{(5,9)} \\
\text { symm. } & & K_{3}^{(9,9)}
\end{array}\right] \text { and }\left[K_{3 v}\right]=\left[\begin{array}{lll}
K_{3}^{(2,2)} & K_{3}^{(2,6)} & K_{3}^{(2,10)} \\
& K_{3}^{(6,6)} & K_{3}^{(6.10)} \\
\text { symm. } & & K_{3}^{(10.10)}
\end{array}\right]
$$

The element matrix $\left[M_{b}\right]$ for the vibration problem is shown next. The elements of the matrix have been defined in Appendix A.

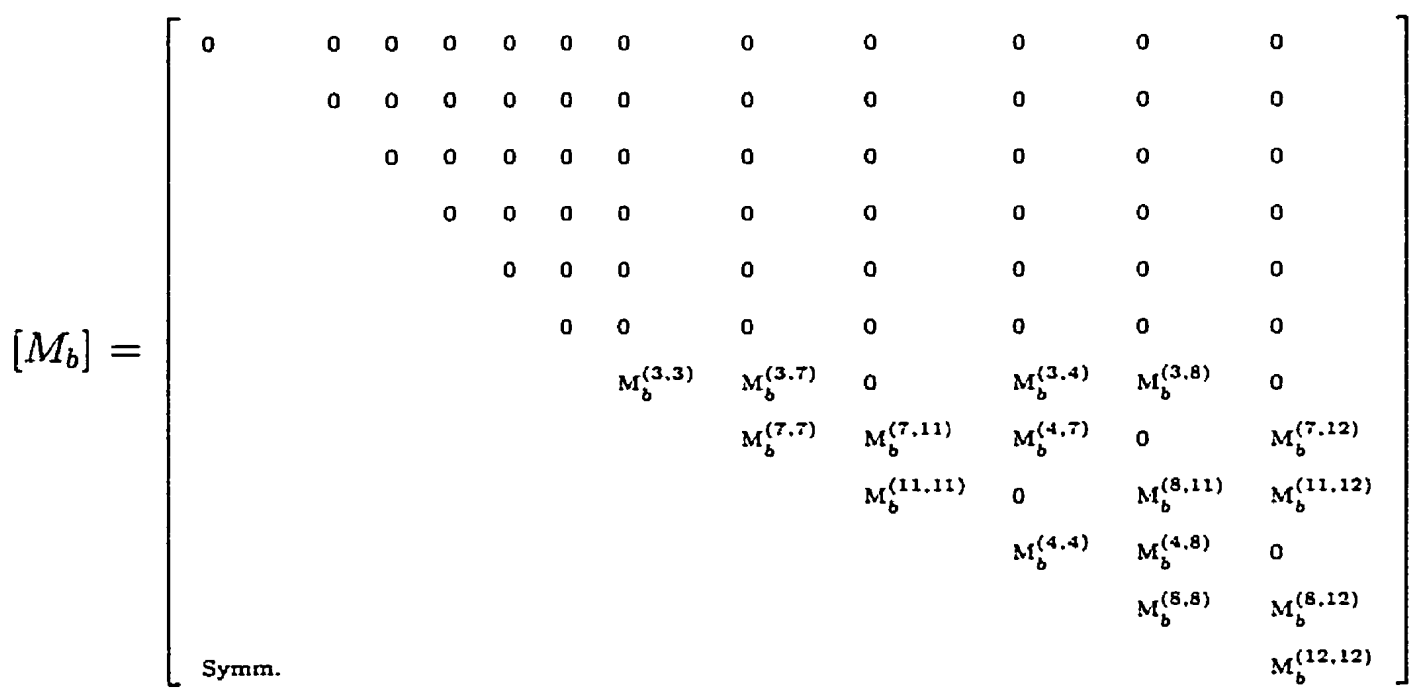

Matrix $\left[M_{b \alpha}\right]$ is comprised of one non-zero submatrix which we will define as

$$
\left[M_{b \alpha}\right]=\left[\begin{array}{llllll}
\mathrm{M}_{b}^{(3,3)} & \mathrm{M}_{b}^{(3,7)} & 0 & \mathrm{M}_{b}^{(3,4)} & \mathrm{M}_{b}^{(3,8)} & 0 \\
& \mathrm{M}_{b}^{(7,7)} & \mathrm{M}_{b}^{(7,11)} & \mathrm{M}_{b}^{(4,7)} & 0 & \mathrm{M}_{b}^{(7,12)} \\
& & \mathrm{M}_{b}^{(11,11)} & 0 & \mathrm{M}_{b}^{(8,11)} & \mathrm{M}_{b}^{(11.12)} \\
& & & \mathrm{M}_{b}^{(4,4)} & \mathrm{M}_{b}^{(4,8)} & 0 \\
& & & & \mathrm{M}_{b}^{(8,8)} & \mathrm{M}_{b}^{(8,12)} \\
\text { Symm. } & & & & & \mathrm{M}_{b}^{(12.12)}
\end{array}\right]
$$

The element matrix $\left[E_{1}\right]$ for the vibration problem is shown next. The elements of the matrix have been defined in Appendix A. 


$$
\left[E_{1}\right]=\left[\begin{array}{lllllllllllll}
0 & 0 & 0 & 0 & 0 & 0 & 0 & 0 & 0 & 0 & 0 & 0 \\
& 0 & 0 & 0 & 0 & 0 & 0 & 0 & 0 & 0 & 0 & 0 \\
& & 0 & 0 & 0 & 0 & 0 & 0 & 0 & 0 & 0 & 0 \\
& & & 0 & 0 & 0 & 0 & 0 & 0 & 0 & 0 & 0 \\
& & & 0 & 0 & 0 & 0 & 0 & 0 & 0 & 0 \\
& & & & 0 & 0 & 0 & 0 & 0 & 0 & 0 \\
& & & & & E_{1}^{(3.3)} & E_{1}^{(3.7)} & 0 & E_{1}^{(3.4)} & E_{1}^{(3.8)} & 0 \\
& & & & & & E_{1}^{(7.7)} & E_{1}^{(7.11)} & E_{1}^{(4.7)} & 0 & E_{1}^{(j .12)} \\
& & & & & & & E_{1}^{(11.11)} & 0 & E_{1}^{(8.11)} & E_{1}^{(13.12)} \\
& & & & & & & & E_{1}^{(4.4)} & E_{1}^{(4.8)} & 0 \\
& & & & & & & & & E_{1}^{(8.8)} & E_{1}^{(8.12)} \\
& & & & & & & & & & E_{1}^{(12.12)}
\end{array}\right]
$$

Matrix $\left[E_{1}\right]$ is comprised of one non-zero submatrix which we will define as

$$
\left[E_{1 \alpha}\right]=\left[\begin{array}{llllll}
E_{1}^{(3,3)} & E_{1}^{(3,7)} & 0 & E_{1}^{(3,4)} & E_{1}^{(3,8)} & 0 \\
& E_{1}^{(7,7)} & E_{1}^{(7,11)} & E_{1}^{(4,7)} & 0 & E_{1}^{(7,12)} \\
& & E_{1}^{(11,11)} & 0 & E_{1}^{(8,11)} & E_{1}^{(11,12)} \\
& & & E_{1}^{(4,4)} & E_{1}^{(4,8)} & 0 \\
& & & & E_{1}^{(8,8)} & E_{1}^{(8,12)} \\
\text { symm. } & & & & & E_{1}^{(12,12)}
\end{array}\right]
$$

The element matrix $\left[E_{3}\right]$ for the vibration problem is shown next. The elements of the matrix have been defined in Appendix A.

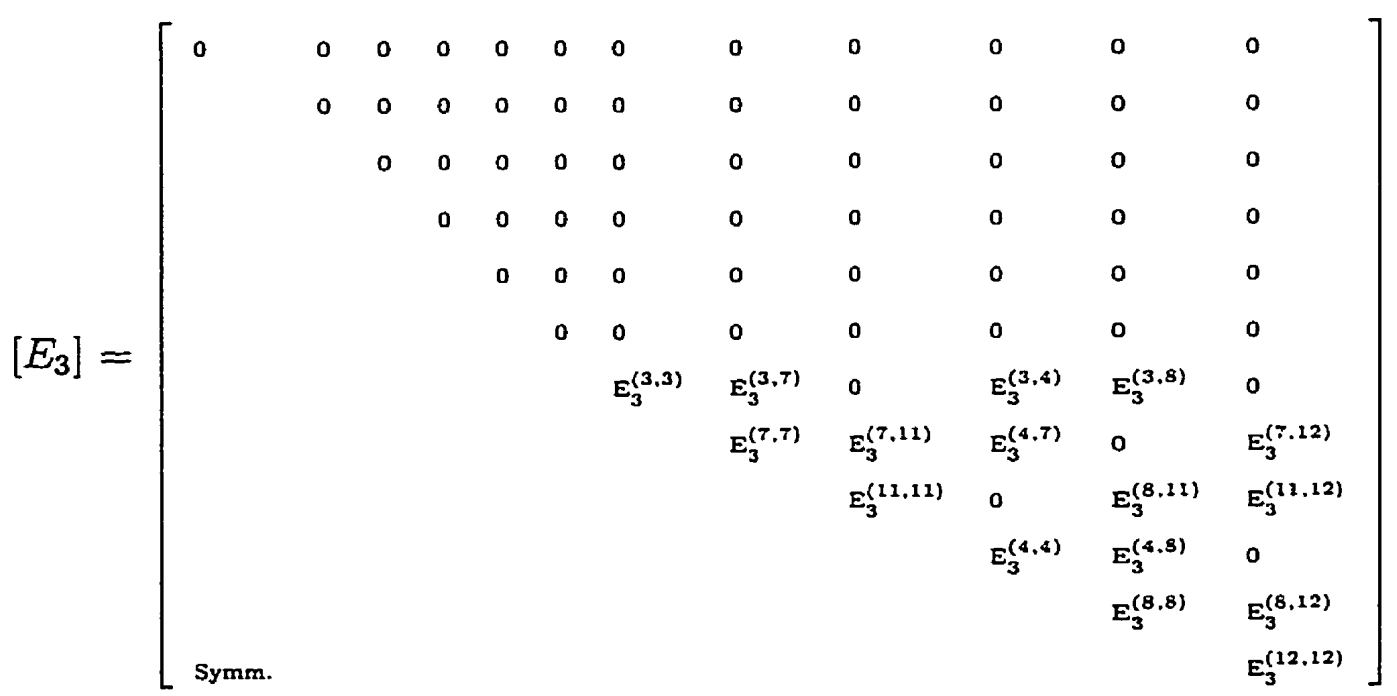

Matrix $\left[E_{3}\right]$ is comprised of one non-zero submatrix which we will define as 


$$
\left[E_{3 \alpha}\right]=\left[\begin{array}{llllll}
E_{3}^{(3,3)} & E_{3}^{(3,7)} & 0 & E_{3}^{(3,4)} & E_{3}^{(3,8)} & 0 \\
& E_{3}^{(7,7)} & E_{3}^{(7.11)} & E_{3}^{(4.7)} & 0 & E_{3}^{(7,12)} \\
& & E_{3}^{(11.11)} & 0 & E_{3}^{(8,11)} & E_{3}^{(11,12)} \\
& & & E_{3}^{(4.4)} & E_{3}^{(4.8)} & 0 \\
& & & & E_{3}^{(8,8)} & E_{3}^{(8,12)} \\
\text { Symm. } & & & & & E_{3}^{(12,12)}
\end{array}\right]
$$

The element matrix $\left[E_{5}\right]$ for the vibration problem is shown next. The elements of the matrix have been defined in Appendix A.

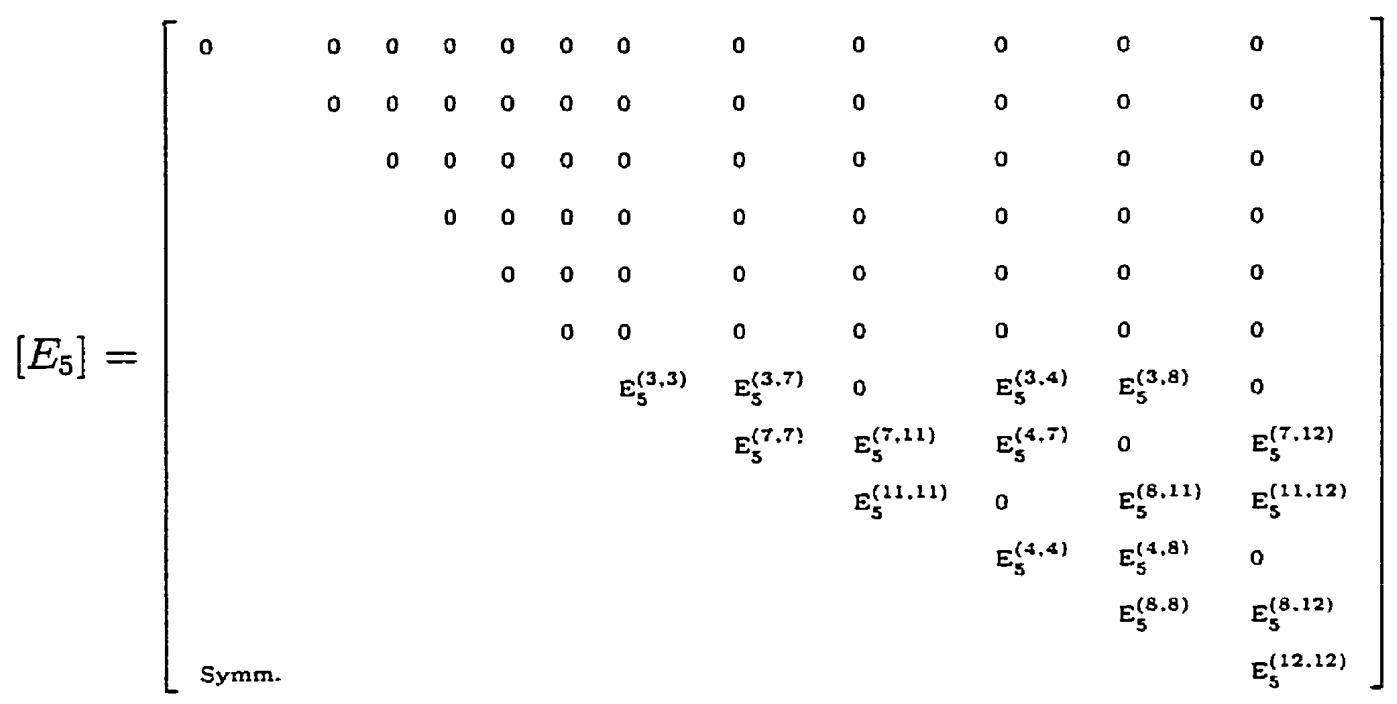

Matrix $\left[E_{5}\right]$ is comprised of one non-zero submatrix which we will define as

$$
\left[E_{5 \alpha}\right]=\left[\begin{array}{llllll}
E_{5}^{(3,3)} & E_{s}^{(3.7)} & 0 & E_{s}^{(3,4)} & E_{s}^{(3,8)} & 0 \\
& E_{s}^{(7.7)} & E_{s}^{(7,11)} & E_{s}^{(4,7)} & 0 & E_{s}^{(7,12)} \\
& & E_{s}^{(11,12)} & 0 & E_{s}^{(8,11)} & E_{s}^{(11,12)} \\
& & & E_{5}^{(4.4)} & E_{s}^{(4,8)} & 0 \\
& & & & E_{s}^{(8,8)} & E_{s}^{(8,12)} \\
\text { Symm. } & & & & & E_{s}^{(12,12)}
\end{array}\right] .
$$




\section{Appendix D}

\section{D RR Vibration of Simply}

\section{Supported Members}

\section{D.1 Introduction}

In this section the element equations created earlier using the three-dimensional stiffness approximation will be modified from their original form to model the vibration of thin-walled structural members simply supported at their ends.

\section{D.2 Formulation}

This problem is an extension of the three-dimension wave propagation problem derived earlier. In the wave propagation problem it is assumed that free-free conditions exist at the end supports. To model simple supports at the ends of the beam there are only a few slight modifications. For simple supports it is necessary that the displacements $u(0) \neq 0, u(a) \neq 0, v(0)=v(a)=w(0)=w(a)=0$. The vibration is assumed to be sinusoidal therefore

$$
\begin{aligned}
u(x) & =\cos \lambda x \\
v(x) & =\sin \lambda x \\
w(x) & =\sin \lambda x
\end{aligned}
$$


where $\lambda=\frac{m \pi}{a}$, and $a$ is the length between supports and $m$ is the number of half wave lengths, i.e. $m=1,2,3 \ldots$. Thus we assume that

$$
q_{u}(x, t)=\left\{\begin{array}{c}
u_{1}(x, t) \\
u_{2}(x, t) \\
: \\
u_{n}(x, t)
\end{array}\right\}=\left\{Q_{0}^{u}\right\} e^{+i \omega t} \cos \lambda x
$$

and

$$
q_{\alpha}(x, t)=\left\{\begin{array}{c}
\alpha_{1}(x, t) \\
\alpha_{2}(x, t) \\
: \\
\alpha_{n}(x, t)
\end{array}\right\}=\left\{Q_{0}^{\alpha}\right\} e^{+i \omega t} \sin \lambda x
$$

where $\alpha=v, w$ and $n$ is the number of nodes in the variable-number-nodes twodimensional element.

Substituting Equations D.4 and D.5 into the element equations derived earlier using Hamilton's principle, Equation 3.27, we get:

$$
\begin{array}{r}
+\omega^{2}[M]\left\{\begin{array}{c}
\cos \lambda x\left\{Q_{0}^{u}\right\} \\
\sin \lambda x\left\{Q_{0}^{v}\right\} \\
\sin \lambda x\left\{Q_{0}^{w}\right\}
\end{array}\right\}+\left[K_{1}\right]\left\{\begin{array}{c}
-\lambda^{2} \cos \lambda x\left\{Q_{0}^{u}\right\} \\
-\lambda^{2} \sin \lambda x\left\{Q_{0}^{v}\right\} \\
-\lambda^{2} \sin \lambda x\left\{Q_{0}^{u}\right\}
\end{array}\right\} \\
+\left[K_{2}\right]\left\{\begin{array}{c}
-\lambda \sin \lambda x\left\{Q_{0}^{u}\right\} \\
\lambda \cos \lambda x\left\{Q_{0}^{v}\right\} \\
\lambda \cos \lambda x\left\{Q_{0}^{w}\right\}
\end{array}\right\}-\left[K_{3}\right]\left\{\begin{array}{c}
\cos \lambda x\left\{Q_{0}^{u}\right\} \\
\sin \lambda x\left\{Q_{0}^{v}\right\} \\
\sin \lambda x\left\{Q_{0}^{w}\right\}
\end{array}\right\}=\left\{\begin{array}{l}
\{0\} \\
\{0\} \\
\{0\}
\end{array}\right\}
\end{array}
$$

where

$$
\begin{gathered}
{[M]=\left[\begin{array}{ccc}
{\left[M_{u}\right]} & 0 & 0 \\
0 & {\left[M_{v_{1}}\right]} & {\left[M_{w_{1}}\right]} \\
0 & {\left[M_{v_{2}}\right]} & {\left[M_{w_{2}}\right]}
\end{array}\right]} \\
{\left[K_{1}\right]=\left[\begin{array}{ccc}
{\left[K_{1 u}\right]} & 0 & 0 \\
0 & {\left[K_{1 v_{1}}\right]} & {\left[K_{1 w_{1}}\right]} \\
0 & {\left[K_{1 v_{2}}\right]} & {\left[K_{1 w_{2}}\right]}
\end{array}\right]} \\
{\left[K_{2}\right]=\left[\begin{array}{ccc}
0 & {\left[K_{2 v}\right]} & {\left[K_{2 w}\right]} \\
{\left[K_{2 u_{1}}\right]} & 0 & 0 \\
{\left[K_{2 u_{2}}\right]} & 0 & 0
\end{array}\right]}
\end{gathered}
$$




$$
\left[K_{3}\right]=\left[\begin{array}{ccc}
{\left[K_{3 u}\right]} & 0 & 0 \\
0 & {\left[K_{3 v_{1}}\right]} & {\left[K_{3 w_{1}}\right]} \\
0 & {\left[K_{3 v_{2}}\right]} & {\left[K_{3 w_{2}}\right]}
\end{array}\right]
$$

Expanding Equation D.6 results in Equations D.11, D.12 and D.13:

$$
\begin{aligned}
& +\omega^{2}\left[M_{u}\right]\left\{Q_{0}^{u}\right\}-\lambda^{2}\left[K_{1 u}\right]\left\{Q_{0}^{u}\right\}+\lambda\left[K_{2 v_{1}}\right]\left\{Q_{0}^{v}\right\} \\
& +\lambda\left[K_{2 v_{2}}\right]\left\{Q_{0}^{w}\right\}-\left[K_{3 u}\right]\left\{Q_{0}^{u}\right\}=\{0\} \\
& +\omega^{2}\left[M_{v_{1}}\right]\left\{Q_{0}^{v}\right\}+\omega^{2}\left[M_{w_{1}}\right]\left\{Q_{0}^{w}\right\}-\lambda^{2}\left[K_{1 v_{1}}\right]\left\{Q_{0}^{v}\right\}-\lambda^{2}\left[K_{1 w_{1}}\right]\left\{Q_{0}^{w}\right\} \\
& -\lambda\left[K_{2 u_{1}}\right]\left\{Q_{0}^{u}\right\}-\left[K_{3 v_{1}}\right]\left\{Q_{0}^{v}\right\}-\left[K_{3 w_{1}}\right]\left\{Q_{0}^{w}\right\}=\{0\} \\
& +\omega^{2}\left[M_{v_{2}}\right]\left\{Q_{0}^{v}\right\}+\omega^{2}\left[M_{w_{2}}\right]\left\{Q_{0}^{w}\right\}-\lambda^{2}\left[K_{1 v_{2}}\right]\left\{Q_{0}^{v}\right\}-\lambda^{2}\left[K_{1 w_{2}}\right]\left\{Q_{0}^{w}\right\} \\
& -\lambda\left[K_{2 u_{2}}\right]\left\{Q_{0}^{u}\right\}-\left[K_{3 v_{2}}\right]\left\{Q_{0}^{v}\right\}-\left[K_{3 w_{2}}\right]\left\{Q_{0}^{w}\right\}=\{0\} .
\end{aligned}
$$

Arranging the four equations into a convenient matrix form

$$
\left[\begin{array}{lll}
{[1]} & {[2]} & {[3]} \\
{[4]} & {[5]} & {[6]} \\
{[7]} & {[8]} & {[9]}
\end{array}\right]\left\{\begin{array}{l}
\left\{Q_{0}^{u}\right\} \\
\left\{Q_{0}^{v}\right\} \\
\left\{Q_{0}^{w}\right\}
\end{array}\right\}=\left\{\begin{array}{l}
\{0\} \\
\{0\} \\
\{0\}
\end{array}\right\}
$$

where

$$
\begin{aligned}
& {[1]=+\omega^{2}\left[M_{u}\right]-\lambda^{2}\left[K_{1 u}\right]-\left[K_{3 u}\right]} \\
& {[2]=+\lambda\left[K_{2 v_{1}}\right]} \\
& {[3]=+\lambda\left[K_{2 v_{2}}\right]} \\
& {[4]=-\lambda\left[K_{2 u_{1}}\right]} \\
& {[5]=+\omega^{2}\left[M_{v 1}\right]-\lambda^{2}\left[K_{1 v_{1}}\right]-\left[K_{3 v_{1}}\right]} \\
& {[6]=+\omega^{2}\left[M_{w 1}\right]-\lambda^{2}\left[K_{1 v_{1}}\right]-\left[K_{3 v_{1}}\right]} \\
& {[7]=-\lambda\left[K_{2 u_{2}}\right]} \\
& {[8]=+\omega^{2}\left[M_{v 2}\right]-\lambda^{2}\left[K_{1 v_{2}}\right]-\left[K_{3 v_{2}}\right]} \\
& {[9]=+\omega^{2}\left[M_{w 2}\right]-\lambda^{2}\left[K_{1 v_{2}}\right]-\left[K_{3 v_{2}}\right]}
\end{aligned}
$$




\section{D.3 Eigenvalue Problem}

The global element equations, Equation D.14, are then assembled to obtain the global structure equations, Equation D.15, in the form $[\mathbf{K}]\left\{\mathbf{Q}_{\mathbf{0}}^{\mathbf{g}}\right\}=\{\mathbf{0}\}$, as

$$
\left[\begin{array}{lll}
{[1]} & {[2]} & {[3]} \\
{[4]} & {[5]} & {[6]} \\
{[7]} & {[8]} & {[9]}
\end{array}\right]\left\{\begin{array}{l}
\left\{\mathbf{Q}_{0}^{\mathbf{u g}}\right\} \\
\left\{\mathbf{Q}_{0}^{\mathrm{vg}}\right\} \\
\left\{\mathbf{Q}_{0}^{\mathbf{w g}}\right\}
\end{array}\right\}=\left\{\begin{array}{l}
\{0\} \\
\{0\} \\
\{0\}
\end{array}\right\}
$$

We can arrange Equation D.15 into the eigenvalue problem below:

$$
\left[\begin{array}{lll}
{[10]} & {[11]} & {[12]} \\
{[13]} & {[14]} & {[15]} \\
{[16]} & {[17]} & {[18]}
\end{array}\right]\left\{\begin{array}{l}
\left\{\mathbf{Q}_{0}^{\text {ug }}\right\} \\
\left\{\mathbf{Q}_{0}^{\text {vg }}\right\} \\
\left\{\mathbf{Q}_{0}^{\mathbf{w g}}\right\}
\end{array}\right\}=\omega^{2}\left[\begin{array}{ccc}
{[19]} & 0 & 0 \\
0 & {[20]} & {[21]} \\
0 & {[22]} & {[23]}
\end{array}\right]\left\{\begin{array}{l}
\left\{\mathbf{Q}_{0}^{\mathrm{ug}}\right\} \\
\left\{\mathbf{Q}_{0}^{\mathbf{v g}}\right\} \\
\left\{\mathbf{Q}_{0}^{\mathbf{w g}}\right\}
\end{array}\right\}
$$

where

$$
\begin{aligned}
& {[10]=-\lambda^{2}\left[K_{1 u}\right]-\left[K_{3 u}\right]} \\
& {[11]=+\lambda\left[K_{2 v_{1}}\right]} \\
& {[12]=+\lambda\left[K_{2 v_{2}}\right]} \\
& {[13]=-\lambda\left[K_{2 u_{1}}\right]} \\
& {[14]=-\lambda^{2}\left[K_{1 v_{1}}\right]-\left[K_{3 v_{1}}\right]} \\
& {[15]=-\lambda^{2}\left[K_{1 v_{1}}\right]-\left[K_{3 v_{1}}\right]} \\
& {[16]=-\lambda\left[K_{2 u_{2}}\right]} \\
& {[17]=-\lambda^{2}\left[K_{1 v_{2}}\right]-\left[K_{3 v_{2}}\right]} \\
& {[18]=-\lambda^{2}\left[K_{1 v_{2}}\right]-\left[K_{3 v_{2}}\right]} \\
& {[19]=-\omega^{2}\left[M_{u}\right]} \\
& {[20]=-\omega^{2}\left[M_{v 1}\right]} \\
& {[21]=-\omega^{2}\left[M_{w 1}\right]} \\
& {[22]=-\omega^{2}\left[M_{v 2}\right]} \\
& {[23]=-\omega^{2}\left[M_{w 2}\right] .}
\end{aligned}
$$

From this eigenvalue problem frequencies, $\omega$ are obtained for a given number of half wavelengths, $m$.

\section{D.4 Summary}

The accuracy of the three-dimension model of the vibration of thin-walled structural members simply supported at their ends is discussed in Chapter 5. The three- 
dimension approximation will be compared to a similar approximation based on twodimensional theory as well as a simplified free vibration model. 


\section{Appendix E}

\section{Coefficients of Matrix $[P]$}

The elements of the matrix $[P]$ appearing in Equation 4.14 are given below:

where

$$
[P]=\left[\begin{array}{llll}
p_{11} & p_{12} & p_{13} & p_{14} \\
p_{21} & p_{22} & p_{23} & p_{24} \\
p_{31} & p_{32} & p_{33} & p_{43} \\
p_{41} & p_{42} & p_{43} & p_{44}
\end{array}\right]
$$

$$
\begin{aligned}
& p_{11}=\frac{D_{66}}{\Delta_{1}} j k\left[d \cos \left(2 h r_{1}\right)-\operatorname{cbcos}\left(2 h r_{2}\right)\right] \\
& p_{21}=\frac{D_{66}}{\Delta_{1}}\left[-r_{1} a_{1} d \sin \left(2 h r_{1}\right)+r_{2} \operatorname{csin}\left(2 h r_{2}\right)\right] \\
& p_{31}=\frac{D_{66}^{2}}{\Delta_{1}} c d\left[\cos \left(2 h r_{1}\right)-\cos \left(2 h r_{2}\right)\right] \\
& p_{41}=\frac{D_{66}^{2}}{\Delta_{1}} j k\left[-r_{1} d\left(1+a_{1}\right) \sin \left(2 h r_{1}\right)+r_{2} c\left(1+b_{1}\right) \sin \left(2 h r_{2}\right)\right] \\
& p_{12}=\frac{D_{66}}{\Delta_{2}} k^{2}\left[-r_{2}\left(1+b_{1}\right) \sin \left(2 h r_{1}\right)+b_{1} r_{1}\left(1+a_{1}\right) \sin \left(2 h r_{2}\right)\right] \\
& p_{22}=\frac{D_{66}}{\Delta_{2}} j k r_{1} r_{2}\left[a_{1}\left(1+b_{1}\right) \cos \left(2 h r_{1}\right)-\left(1+a_{1}\right) \cos \left(2 h r_{2}\right)\right] \\
& p_{32}=\frac{D_{\delta_{6}}^{2}}{\Delta_{2}} j k\left[c r_{2}\left(1+b_{1}\right) \sin \left(2 h r_{1}\right)+-d r_{1}\left(1+a_{1}\right) \sin \left(2 h r_{2}\right)\right] \\
& p_{42}=\frac{D_{66}^{2}}{\Delta_{2}} k^{2} r_{1} r_{2}\left(1+a_{1}\right)\left(1+b_{1}\right)\left[-\cos \left(2 h r_{1}\right)+\cos \left(2 h r_{2}\right)\right] \\
& p_{13}=\frac{b_{1} k^{2}}{\Delta_{1}}\left[\cos \left(2 h r_{1}\right)-\cos \left(2 h r_{2}\right)\right] \\
& p_{23}=\frac{j k}{\Delta_{1}}\left[r_{1} a_{1} b_{1} \sin \left(2 h r_{1}\right)-r_{2} \sin \left(2 h r_{2}\right)\right]
\end{aligned}
$$




$$
\begin{aligned}
p_{33} & =\frac{D_{66}}{\Delta_{1}} j k\left[-b_{1} \cos \left(2 h r_{1}\right)+d \cos \left(2 h r_{2}\right)\right] \\
p_{43} & =\frac{D_{66}}{\Delta_{1}} k^{2}\left[-r_{1} b_{1}\left(1+a_{1}\right) \sin \left(2 h r_{1}\right)+r_{2}\left(1+b_{1}\right) \sin \left(2 h r_{2}\right)\right] \\
p_{14} & =\frac{j k}{\Delta_{2}}\left[-r_{2} \sin \left(2 h r_{1}\right)-a_{1} b_{1} r_{1} \sin \left(2 h r_{2}\right)\right] \\
p_{24} & =\frac{a_{1}}{\Delta_{2}} r_{1} r_{2}\left[-\cos \left(2 h r_{1}\right)+\cos \left(2 h r_{2}\right)\right] \\
p_{43} & =\frac{D_{66}}{\Delta_{2}}\left[-r_{2} \operatorname{csin}\left(2 h r_{1}\right)+r_{1} a_{1} d \sin \left(2 h r_{2}\right)\right] \\
p_{44} & =\frac{D_{66}}{\Delta_{2}} j k r_{1} r_{2}\left[-\left(1+a_{1}\right) \cos \left(2 h r_{1}\right)+a_{1}\left(1+b_{1}\right) \sin \left(2 h r_{2}\right)\right] \\
c & =(1-\bar{\delta}) k^{2}-\bar{\beta} r_{1}^{2} a_{1} ; \quad d=(1-\bar{\delta}) k^{2} b_{1}-\bar{\beta} r_{2}^{2}, \\
\Delta_{1} & =D_{66} j k \bar{\beta} r_{2}^{2}\left(\frac{r_{1}^{2}}{r_{2}^{2}} a_{1} b_{1}-1\right) ; \quad \Delta_{2}=D_{66} j k r_{1} r_{2}\left(a_{1} b_{1}-1\right), \\
h & =y_{i+1}-y_{i}=h_{i} .
\end{aligned}
$$

